\title{
Tabulation of \\ Voluntary Standards and Certification Programs for Consumer Products
}

U.S. EPARTMENT OF COMMERCE

National Bureau of Standards 
The National Bureau of Standards ${ }^{1}$ was established by an act of Congress March 3 , 1901. The Bureau's overall goal is to strengthen and advance the Nation's science and technology and facilitate their effective application for public benefit. To this end, the Bureau conducts research and provides: (1) a basis for the Nation's physical measurement system, (2) scientific and technological services for industry and government, (3) a technical basis for equity in trade, and (4) technical services to promote public safety. The Bureau consists of the Institute for Basic Standards, the Institute for Materials Research, the Institute for Applied Technology, the Center for Computer Sciences and Technology, and the Office for Information Programs.

THE INSTITUTE FOR BASIC STANDARDS provides the central basis within the United States of a complete and consistent system of physical measurement; coordinates that system with measurement systems of other nations; and furnishes essential services leading to accurate and uniform physical measurements throughout the Nation's scientific community, industry, and commerce. The Institute consists of a Center for Radiation Research, an Office of Measurement Services and the following divisions:

Applied Mathematics - Electricity - Mechanics - Heat — Optical Physics — Linac Radiation" - Nuclear Radiation " - Applied Radiation — Quantum Electronics : - Electromagnetics : — Time and Frequency " - Laboratory Astrophysics : - Cryogenics "

THE INSTITUTE FOR MATERIALS RESEARCH conducts materials research leading to improved methods of measurement, standards, and data on the properties of well-characterized materials needed by industry, commerce, educational institutions, and Government; provides advisory and research services to other Government agencies; and develops, produces, and distributes standard reference materials. The Institute consists of the Office of Standard Reference Materials and the following divisions:

Analytical Chemistry-Polymers-Metallurgy-Inorganic Materials-Reactor Radiation-Physical Chemistry.

THE INSTITUTE FOR APPLIED TECHNOLOGY provides technical services to promote the use of available technology and to facilitate technological innovation in industry and Government; cooperates with public and private organizations leading to the development of technological standards (including mandatory safety standards), codes and methods of test; and provides technical advice and services to Government agencies upon request. The Institute also monitors NBS engineering standards activities and provides liaison between NBS and national and international engineering standards bodies. The Institute consists of a Center for Building Technology and the following divisions and offices:

Engineering and Product Standards-Weights and Measures-Invention and Innovation-Product Evaluation Technology-Electronic Technology-Technical Analysis-Measurement Engineering-Building Standards and Code Services $^{4}$-Housing Technology ${ }^{4}$ - Federal Building Technology ${ }^{4}$-Structures, Materials and Life Safety ${ }^{4}$-Building Environment ${ }^{4}$-Technical Evaluation and Application $^{4}$-Fire Technology.

THE INSTITUTE FOR COMPUTER SCIENCES AND TECHNOLOGY conducts research and provides technical services designed to aid Government agencies in improving cost effectiveness in the conduct of their programs through the selection, acquisition, and effective utilization of automatic data processing equipment; and serves as the principal focus within the executive branch for the development of Federal standards for automatic data processing equipment, techniques, and computer languages. The Center consists of the following offices and divisions:

Information Processing Standards-Computer Information-Computer Services - Systems Development-Information Processing Technology.

THE OFFICE FOR INFORMATION PROGRAMS promotes optimum dissemination and accessibility of scientific information generated within NBS and other agencies of the Federal Government; promotes the development of the National Standard Reference Data System and a system of information analysis centers dealing with the broader aspects of the National Measurement System; provides appropriate services to ensure that the NBS staff has optimum accessibility to the scientific information of the world, and directs the public information activities of the Bureau. The Office consists of the following organizational units:

Office of Standard Reference Data-Office of Technical Information and Publications-Library-Office of International Relations.

\footnotetext{
- Headquarters and Laboratories at Gaithersburg, Maryiand, unless otherwise noted; mailing address Washington, D.C. 20234.

2 Part of the Center for Radiation Research.

3 Located at Boufder, Colorado 50302.

- Part of the Center for Bullding l'echuology.
} 


\section{Tabulation of Voluntary Standards and Certification Programs for Consumer Products}

Sophie J. Chumas

Standards Information Services

Engineering and Product Standards Division

Institute for Applied Technology

National Bureau of Standards

Washington, D.C. 20234

NBS Technical Notes are designed to supplement the Bureau's regular publications program. They provide a means for making available scientific data that are of transient or limited interest. Technical Notes may be listed or referred to in the open literature.

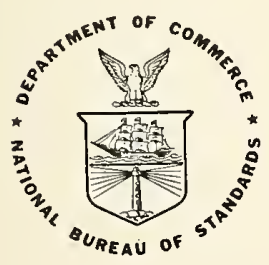

U.S. DEPARTMENT OF COMMERCE, Frederick B. Dent, Secretary NATIONAL BUREAU OF STANDARDS, Richard W. Roberts, Director 
National Bureau of Standards Technical Note 762

Nat. Bur. Stand. (U.S.), Tech. Note 762119 pages (Mar. 1973) CODEN: NBTNAE

(Supersedes TN 705 issued Dec. 1971)

For sole by the Superintendent of Documents, U.S. Government Printing Office, Washington, D.C., 20402. Price \$1.25 domestic postpaid or \$1.00 G.P.O. Bookstore. 


\section{Contents}

1. Introduction . . . . . . . . . . . . . . Page

2. Product Categories . . . . . . . . . . . . . 3

3. Tabulation ................. . . . 4 4

4. Indexes . . . . . . . . . . . . . . . 53

4.1. Standards Titles . . . . . . . . . . 53

4.2. Product Index Including SIC Numbers . . . . . . 72

4.3. Numerical Index of Products . . . . . . . . . 95

4.4. Index of Associations . . . . . . . . . . 106

4.4.1. Alphabetical by Acronym . . . . . . 106

4.4.2. Alphabetical by Association . . . . . 108 

Tabulation of Voluntary Standards

and Certification Programs for Consumer Products

\author{
Sophie J. Chumas
}

This tabulation is a revised and enlarged version of NBS Technical Note 705, "Tabulation of Voluntary National Standards, Industry Standards, International Recomendations and Certification Programs for Consumer Products," issued December 1971.

Technical Note 705 was a revision of the Draft Tabulation compiled by the American National Standards Institute and issued in April 1970. The categories and products covered in the tabulation were originally based on those listed in the Consumer Product Safety Index (CPSI) of the National Commission on Product Safety, published in July 1970. The products and categories in the current revision are based on those developed for the National Electronic Injury Surveillance System (NEISS) of the Food and Drug Administration. The NEISS listings are an expansion and revision of the CPSI. NEISS product areas were slightly altered to conform to the scope of this tabulation.

The tabulation lists over 700 product areas and over 1000 standards titles covering products found in and around the home. (The major consumer product areas not included are foods, beverages, and drugs.) The tabulation also indicates the applicable voluntary national, industrial, and international standards which deal primarily with either safety or performance or both aspects of the products listed. For some of the product areas, there are no applicable standards. Available information on certification programs and standards under development, and the Standard Industrial Classification (SIC) numbers for the products are also provided.

Key Words: Certification programs; consumer products; household products; industry standards; international recommendations; national standards; product standards; recommended practices; specifications; test methods.

\title{
1. Introduction
}

This tabulation is a revised and enlarged version of NBS Technical Note 705, "Tabulation of Voluntary National Standards, Industry Standards, International Recommendations and Certification Programs for Consumer Products," issued December 1971.

The original draft of the tabulation was developed by the Consumer Council of the American National Standards Institute (ANSI) in 1970. The categories and products covered in the ANSI tabulation were based on those listed in the Consumer Product Safety Index (CPSI) of the National Comission on Product Safety, issued in July 1970. In 1971, at the Consumer Council's request, the Bureau's Office of Engineering and Information Processing Standards revised the draft tabulation and issued it as NBS Technical Note 705 .

The current revision of Technical Note 705 covers products and categories taken from the National Electronic Injury Surveillance System (NEISS) of the Food and Drug Administration. The NEISS listings are an expansion and revision of the CPSI. NEISS product areas were slightly altered to conform to the scope of this tabulation. The omitted product numbers of the tabulation are: 0336, 0641, 0656, 0914, $1004-1016,1268,1270,1630,1640,1813$.

Although its principal objective is to indicate existing standards and certification programs for consumer products found in and around the home, this revision includes for the first time available information on standards under development, the Standard Industrial Classification (SIC) number of the listed products, and a listing of the standards titles.

For the purpose of this tabulation the word "standards" encompasses specifications, recommended practices, analyses, and selected test methods for products or certain aspects of the products, but does not include those standards related to installation, maintenance, definitions, or parts of products.

The certification programs listed are (1) Association certification certification programs, (2) thirdparty certification programs or (3) self-certification programs.

Information on standards which have not yet reached the publication stage is indicated by "IP" (in process) and appears under the column "Latest Issue or Status of Standard." Tentative standards are indicated by "T" in the same column, after the date. Within the tables the numbers listed under the heading "Product Number" are those used by NEISS. The product number should always be preceded by the 
letter "P" when cited in the literature (e.g., P0213, P1620). In the column headed "Responsible Organization and Standard Designation," the symbol $\subset$ means that information is contained in the standard which does not relate exclusively the the product. An asterisk (*) preceding a number means that the number was assigned as a retrieval designation by Standards Information Services in the absence of an official number assigned by the issuing organization. The letters listed under "Type of Standard" are keyed as follows:

$$
\begin{aligned}
0 & =\text { Other } \\
P & =\text { Performance } \\
S & =\text { Safety } \\
T M & =\text { Test Method }
\end{aligned}
$$

The tabulation is expected to be of use to standards-writing organizations, Federal and State government agencies, distribution and retail groups, labor unions and trade association, consumer organizations, and individual consumers. It is hoped that the information contained in this tabulation will indicate those product areas where standards are needed, reflect possible duplication of effort by standards-writing organizations, and provide an impetus for coordination of standardization activities.

Standards Information Services will continue to keep abreast of standards development and publication, and, as sufficient changes occur to so warrant, new editions of this tabulation will be published. Users are invited to make comments or provide information which can be considered for incorporation in the next edition, so that it will more completely define the status of consumer product standards. There is a particular need for information on standards in the process of development since the only way to obtain this information is directly from the Association involved. Send comments to Standards Information Services, National Bureau of Standards, Technology Building, Room Bl47, Washington, D.C. 20234. 


\section{PRODUCT CATEGORIES}

Product No. General Household Appliances . . . . . . . . . . . . . . . . 0101 - 0131 Kitchen Appliances . . . . . . . . . . . . . . . . . . $0201-0256$ Space Heating, Cooling and Ventilating Appliances . . . . . . . . . . . . 0301 - 0341

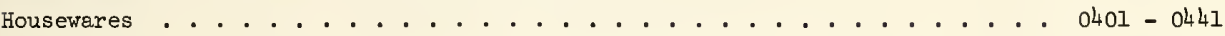
Home Communications, Entertainment, and Hobby Equipment . . . . . . . . 0501 - 0534 Home Furnishings and Fixtures ..................... . $0601-0674$ Home Alarm, Escape, and Protection Devices . . . . . . . . . . . . 0701 - 0707 Home Workshop Apparatus, Tools, and Attachments . . . . . . . . . . . 0801 - 0835 Household Maintenance Products . . . . . . . . . . . . . . 0901 - 0943 Farm Supplies and Equipment . . . . . . . . . . . . . . . . . 1001 - 1003 Packaging and Containers for Household Products . . . . . . . . . . . . . 1101 - 1115 Sports and Recreational Equipment . . . . . . . . . . . . . . . . $1201-1275$ Toys . . . . . . . . . . . . . . . . . . . . . . . . $1301-1368$ Yard and Garden Equipment . . . . . . . . . . . . . . . . . . . 1401 - 1433 Child Nursery Equipment and Supplies . . . . . . . . . . . . . . . . 1501 - 1531 Personal Use Items . . . . . . . . . . . . . . . . . . . $1601-1647$ Products Not Elsewhere Classified . . . . . . . . . . . . . . . 1701 - 1721 Home Structures, Construction Materials . . . . . . . . . . . . . . . 1801 - 1839 


\begin{tabular}{|c|c|c|c|c|c|c|}
\hline $\begin{array}{l}\text { Product } \\
\text { Number }\end{array}$ & $\begin{array}{l}\text { Product } \\
\text { Description }\end{array}$ & $\begin{array}{c}\text { Responsible Organi- } \\
\text { zation and Standard } \\
\text { Designation }\end{array}$ & $\begin{array}{l}\text { Latest } \\
\text { Issue or } \\
\text { Status of } \\
\text { Standard }\end{array}$ & $\begin{array}{l}\text { Standard } \\
\text { Accepted by }\end{array}$ & $\begin{array}{l}\text { Type of } \\
\text { Standard }\end{array}$ & $\begin{array}{l}\text { Certifi- } \\
\text { cation } \\
\text { Program }\end{array}$ \\
\hline \multirow[t]{4}{*}{ P0101 } & \multirow[t]{4}{*}{$\begin{array}{l}\text { GENERAL HOUSEHOLD APPLIANCES } \\
\text { Clothes washers without wringers } \\
\text { or other dryers }\end{array}$} & UL 560 & 1968 & $\begin{array}{l}\text { ANSI C33.13 } \\
(1972)\end{array}$ & $\mathrm{s}$ & UL \\
\hline & & AHAM HLW-1 & 1971 & $\begin{array}{l}\text { ANSI } 2224.1 \\
(1971)\end{array}$ & $P$ & -- \\
\hline & & $\begin{array}{l}\text { ASSE } 1007 \\
\text { CEE } 10, \text { pt.1 and }\end{array}$ & 1971 & -- & $\mathrm{P}$ & ASSE \\
\hline & & $\begin{array}{l}\text { pt.2, sec.J } \\
\text { IEC } 335-1 \& 335-7\end{array}$ & $\begin{array}{l}1964 \& 68 \\
1971\end{array}$ & $\begin{array}{l}-- \\
--\end{array}$ & $\begin{array}{l}\text { SP } \\
\text { S }\end{array}$ & $\begin{array}{l}\mathrm{CEE} \\
--\end{array}$ \\
\hline \multirow[t]{4}{*}{0102} & \multirow[t]{4}{*}{ Washing machines with wringers } & AHAM HLW-1 & 1971 & $\begin{array}{l}\text { ANSI } 2224.1 \\
(1971)\end{array}$ & $\mathrm{P}$ & -- \\
\hline & & $\begin{array}{l}\text { CEE 10, pt.1 and } \\
\text { pt.2, sec.J }\end{array}$ & $1964 \& 68$ & -- & $\mathrm{SP}$ & CEE \\
\hline & & $\begin{array}{l}\text { CCEE } 10 \\
\text { UL } 560\end{array}$ & $\begin{array}{l}1960 \\
1968\end{array}$ & $\begin{array}{l}\text { ANSI } \bar{C} \text { C33.13 } \\
\quad(1972)\end{array}$ & $\begin{array}{l}\mathrm{s} \\
\mathrm{s}\end{array}$ & $\begin{array}{l}\text { CEE } \\
\text { UL }\end{array}$ \\
\hline & & IEC 335-1 \& 335-7 & 1971 & -- & s & -- \\
\hline \multirow[t]{5}{*}{0103} & \multirow[t]{5}{*}{$\begin{array}{l}\text { Washing machines with spin } \\
\text { dryers without thermal dryers }\end{array}$} & UL 560 & 1968 & $\begin{array}{l}\text { ANSI C33.13 } \\
(1968)\end{array}$ & $\mathrm{s}$ & UL \\
\hline & & $\begin{array}{l}\text { ASSE } 1007 \\
\text { CEE 10, pt.1 and }\end{array}$ & 1971 & -- & $P$ & ASSE \\
\hline & & $\begin{array}{l}\mathrm{pt} \cdot 2, \mathrm{sec} \cdot \mathrm{J} \\
\mathrm{CCEE} 10\end{array}$ & $\begin{array}{l}1964868 \\
1960\end{array}$ & -- & $\begin{array}{l}\text { SP } \\
\text { S }\end{array}$ & $\begin{array}{l}\mathrm{CEE} \\
\mathrm{CEE}\end{array}$ \\
\hline & & $\begin{array}{l}\text { IEC } 335-1 \text { \& } 335-7 \\
\text { CEE } 10 \mathrm{pt.1} \text { and }\end{array}$ & 1971 & -- & $\mathrm{S}$ & -- \\
\hline & & $\mathrm{pt} .2$, sec. $\mathrm{K}$ & $1964 \& 68$ & -- & SP & CEE \\
\hline \multirow[t]{3}{*}{0104} & \multirow[t]{3}{*}{$\begin{array}{l}\text { Washing machines with electric } \\
\text { dryers }\end{array}$} & UL 560 & 1968 & $\begin{array}{l}\text { ANSI C33.13 } \\
(1971)\end{array}$ & $\mathrm{s}$ & UL \\
\hline & & ASSE 1007 & 1971 & -- & $\mathrm{P}$ & ASSE \\
\hline & & IEC $335-1 \& 335-7$ & 1971 & -- & $\mathrm{S}$ & -- \\
\hline 0105 & Washing machines with gas aryers & -- & -- & -- & -- & -- \\
\hline \multirow[t]{5}{*}{0106} & \multirow[t]{5}{*}{$\begin{array}{l}\text { Electric dryers without washing } \\
\text { machines attached }\end{array}$} & UL 560 & 1968 & $\begin{array}{l}\text { ANSI C33.13 } \\
(1968)^{13}\end{array}$ & $\mathrm{~s}$ & UL \\
\hline & & ASSE 1007 & 1971 & -- & $\mathrm{P}$ & ASSE \\
\hline & & $\begin{array}{l}\text { AHAM } \\
\operatorname{CEE} 10, \text { pt.1 and }\end{array}$ & IP 1972 & -- & $\mathrm{P}$ & -- \\
\hline & & pt.2, sec.K & 1964868 & -- & SP & CEE \\
\hline & & $\begin{array}{l}\text { CCEE } 10 \\
\text { IEC } 335-1 \text { \& } 335-. .\end{array}$ & IP 1972 & - & s & -- \\
\hline \multirow[t]{2}{*}{0107} & \multirow[t]{2}{*}{ Gas dryers without washing } & & & & & \\
\hline & & $\begin{array}{l}\text { ANSI Z21.5.1 } \\
\text { AHAM }\end{array}$ & $\begin{array}{l}1968 \\
\text { IP } 1972\end{array}$ & $\begin{array}{l}-- \\
--\end{array}$ & $\begin{array}{l}\text { SP } \\
P\end{array}$ & $\begin{array}{l}\text { AGA } \\
--\end{array}$ \\
\hline \multirow[t]{2}{*}{0108} & \multirow[t]{2}{*}{ Ironers, "mangle" } & UL 141 & 1971 & -- & $\mathrm{s}$ & UL \\
\hline & & $\begin{array}{l}\text { (EE } 11, \mathrm{pt} .1 \text { and } \\
\mathrm{pt.2} \text {, sec.G }\end{array}$ & $1964 \& 65$ & -- & SP & CEE \\
\hline
\end{tabular}




\begin{tabular}{|c|c|c|c|c|c|c|}
\hline $\begin{array}{l}\text { Product } \\
\text { Number }\end{array}$ & $\begin{array}{l}\text { Product } \\
\text { Description }\end{array}$ & $\begin{array}{l}\text { Responsible Organi- } \\
\text { zation and Standard } \\
\text { Designation }\end{array}$ & $\begin{array}{l}\text { Latest } \\
\text { Issue or } \\
\text { Status of } \\
\text { Stendard } \\
\end{array}$ & $\begin{array}{l}\text { Standard } \\
\text { Accepted by }\end{array}$ & $\begin{array}{l}\text { Type of } \\
\text { Standard }\end{array}$ & $\begin{array}{l}\text { Certifi- } \\
\text { cation } \\
\text { Program }\end{array}$ \\
\hline \multirow[t]{2}{*}{ P0109 } & Electric blankets & UL 964 & 1968 & $\begin{array}{l}\text { ANSI C33.52 } \\
(1969)\end{array}$ & s & UL \\
\hline & & $\begin{array}{l}\text { AHAM DA2 } \\
\text { IEC } 299\end{array}$ & $\begin{array}{l}1967 \\
1969\end{array}$ & - & $\begin{array}{l}P \\
P\end{array}$ & -- \\
\hline 0110 & Electric heating pads & UL 130 & 1968 & $\begin{array}{l}\text { ANSI C33.7 } \\
\quad(1969)\end{array}$ & $\mathrm{s}$ & UL \\
\hline \multirow[t]{2}{*}{0111} & Electric fans, portable & $\begin{array}{l}\text { IEC } 174 \\
\text { IEC } 175 \\
\text { UL } 507\end{array}$ & $\begin{array}{l}1966 \\
1965 \\
1969\end{array}$ & $\begin{array}{c}\overline{--} \\
\overline{A N S I} \text { C33.54 } \\
(1969)\end{array}$ & $\begin{array}{l}P \\
P \\
S\end{array}$ & $\begin{array}{l}-- \\
\overline{U L}\end{array}$ \\
\hline & & $\begin{array}{l}\text { AHAM } \\
\text { IEC } 342 \\
\text { CCEE } 10\end{array}$ & $\begin{array}{l}\text { IP } 1972 \\
1971 \\
1960\end{array}$ & $\begin{array}{l}-- \\
-- \\
--\end{array}$ & $\begin{array}{l}P \\
S \\
S\end{array}$ & $\begin{array}{l}-- \\
-- \\
\mathrm{CEE}\end{array}$ \\
\hline \multirow[t]{2}{*}{0112} & $\begin{array}{l}\text { Sewing machines and accessories, } \\
\text { including bobbins }\end{array}$ & CUL 73 & 1972 & $\begin{array}{l}\text { ANSI C33.36 } \\
(1972)\end{array}$ & $\mathrm{s}$ & UL \\
\hline & & CCEE 10 & 1960 & -- & s & CEE \\
\hline \multirow[t]{2}{*}{0113} & Floor buffers and waxers & CUL 73 & 1972 & $\begin{array}{l}\text { ANSI C } 33.36 \\
(1972)\end{array}$ & S & UL \\
\hline & & $\begin{array}{l}\text { UL } 561 \\
\text { CCEE } 10 \\
\text { IEC } 369\end{array}$ & $\begin{array}{l}\text { IP } 1971 \\
1960 \\
1971\end{array}$ & $\begin{array}{l}-- \\
--\end{array}$ & $\begin{array}{l}S \\
S \\
P\end{array}$ & $\begin{array}{l}\text { UL } \\
\text { CEE } \\
--\end{array}$ \\
\hline 0114 & Electric rug cleaners/shampooers & $\begin{array}{l}\mathrm{CEE} 10, \mathrm{pt} .1 \text { and } \\
\text { pt.2, sec.A. }\end{array}$ & $1964 \& 65$ & -- & SP & $\mathrm{CEE}$ \\
\hline \multirow[t]{2}{*}{0115} & Vacuum cleaners & $\begin{array}{l}\text { VCMA E1 } \\
\text { CUL } 73\end{array}$ & $\begin{array}{l}1966 \\
1972\end{array}$ & $\underset{(1972)}{\operatorname{ANSI}} \overline{\mathrm{C} 33.36}$ & $\begin{array}{l}P \\
S\end{array}$ & $\overline{U t}$ \\
\hline & & $\begin{array}{l}\text { UL } 1017 \\
\text { IEC } 312 \\
\text { CEE } 10 \text {, pt.1 and } \\
\text { pt.2, sec.A } \\
\text { CCEE } 10 \\
\text { ASTM } \\
\text { IEC } 335-1 \text { \& } 335-2\end{array}$ & $\begin{array}{l}\text { IP } 1971 \\
1969 \\
1964865 \\
1960 \\
\text { IP } 1972 \\
1970\end{array}$ & $\begin{array}{l}-- \\
-- \\
-- \\
-- \\
-- \\
--\end{array}$ & $\begin{array}{l}S \\
P \\
S P \\
S \\
--\end{array}$ & $\begin{array}{l}\text { UL } \\
-- \\
\text { CEE } \\
\text { CEE } \\
-- \\
--\end{array}$ \\
\hline 0116 & Electric sweepers/brooms & -- & - & -- & - & -- \\
\hline 0117 & $\begin{array}{l}\text { Automatic door openers and } \\
\text { closers, automatic garage } \\
\text { doors }\end{array}$ & UL 325 & IP 1972 & -- & 8 & UL \\
\hline 0118 & Gas water heaters & $\begin{array}{l}\text { ANSI } 221.10 .1 \\
\text { ANSI Z21.10.3 }\end{array}$ & $\begin{array}{l}1971 \\
1971\end{array}$ & $\ddot{-+}$ & $\begin{array}{l}\mathrm{S} \\
\mathrm{S}\end{array}$ & $\begin{array}{l}\text { AGA } \\
\text { AGA }\end{array}$ \\
\hline \multirow[t]{3}{*}{0119} & Electric water heaters & UL 174 & 1972 & $\begin{array}{l}\text { ANSI C33.87 } \\
(1972)\end{array}$ & $s$ & UL \\
\hline & & CUL 499 & 1972 & $\begin{array}{l}\text { ANSI C33.11 } \\
(1972)\end{array}$ & $\mathrm{s}$ & UL \\
\hline & & $\begin{array}{l}\text { ANSI C72.1 } \\
\text { CEE } 11, \text { pt.1 and } \\
\text { pt.2, sec.E }\end{array}$ & $\begin{array}{c}1972 \\
1964 \& 66\end{array}$ & -- & $\begin{array}{l}\text { SP } \\
\text { SP }\end{array}$ & CEE \\
\hline 0120 & Oil water heaters & UL 732 & 1969 & -- & s & UL \\
\hline 0121 & $\begin{array}{l}\text { Incinerators without gas or } \\
\text { electric heat supply }\end{array}$ & IIA & IP 1971 & -- & -- & -- \\
\hline 0122 & Electric incinerators & $\begin{array}{l}\text { NFPA } 82 \\
\text { UL } 791\end{array}$ & $\begin{array}{l}1971 \\
1970\end{array}$ & -- & $\begin{array}{l}\text { S } \\
\text { S }\end{array}$ & $\overline{U L}$ \\
\hline
\end{tabular}


IIA *I

UL 791

ANSI $\mathrm{Z} 21.6$

1968

1970

1969
ASHRAE 18

ARI 1010

UL 399
WCF S100

1972

1970

1972

1972

Water softeners and conditioners/ appliance$$
1972
$$

0126 Washing machines not otherwise specified

0127 Clothes aryers not otherwise specified

CEE 11, pt.I and pt. $2, \sec . \mathrm{N}$ IEC 335-1 \& 335-7

CCEE 11, pt.1 and pt.2, sec.B

1966

1964867 1971

0128 Water heaters not otherwise specified

UL 964

AHAM DA2

1968

1967
UL 644

$$
\text { fittings }
$$

gas tanks and

fittings

\section{KITCHEN APPLIANCES}

0201 Electric ranges/stoves with ovens, except self-cleaning ovens

$$
\begin{aligned}
& \text { AHAM ER-1 } \\
& \text { UL } 858 \\
& \text { IEC } 335-6
\end{aligned}
$$

0202 Electric ranges/stoves without

$$
\text { ovens }
$$

UL 858 .

AHAM ER-1

CEE 1l, pt.l and pt.2, sec.A

IEC $335-6$

ANSI Z21.1.I

ANSI Z21.1.2

\section{2 \\ 1969

\begin{tabular}{|c|c|}
\hline-- & $O(T M)$ \\
\hline $\begin{array}{l}\text { ANSI A112.11.1 } \\
(1968)\end{array}$ & PSO \\
\hline $\begin{array}{c}\text { ANSI C33.82 } \\
(1972)\end{array}$ & S \\
\hline
\end{tabular} \\ 1971}

1971

ANSI BI42.I

(1972)
PS

WCF

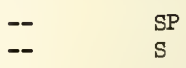

CEE

$-$

CEE

$\begin{array}{cc}\text { ANSI C } 33.52 & \text { S } \\ (1969) & \\ -- & \text { P }\end{array}$

UL

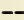

UL
UL

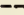

UL

$$
\text { (1969) }
$$

1962

ANSI C71.I

(1972)

$\begin{array}{ll}-- & \text { SP } \\ -- & \text { S }\end{array}$

CEE

1971

S

$\begin{array}{ll}-- & S P \\ -- & S P\end{array}$

AGA AGA 
P0205 Electric ovens separate from ranges, except self-cleaning

AHAM ER-1
CAHAM DA-9
CUL 858
CUL 499
CCEE 11 , pt. 1 and
pt.2, sec.A
CCEE 11, pt. 1 and
pt.2, sec. M
IEC $335-9$
CIEC $335-6$

1962
1964
1969
1972
1964866
1964868
1972
1971

ANSI C71.1
$\quad(1972)$
ANSI C33.53
$\quad(1969)$
ANSI C33.11
$(1972)$

0206 Gas ovens separate from ranges, except self-cleaning ovens 0207 Microwave ovens separate from
ranges

UL 923
IEC
AHAM
IEC $335-.$.

0208 Electric refrigerators

0209 Gas refrigerators

0210 Electric freezers, separate

ASHRAE 15

UL 250

AHAM HRF-1

ISO R824 and R825

ASHRAE 13

CCEE 10

ANSI Z21.19

ISO $R 824$ and $R 825$

UL 250

AHAM HRF-1

ASHRAE 13

CUL 499

AHAM DA-10

0213 Can openers, powered

0214 Dishwashers

0215 Electric blenders
AHAM CO-1

CUL 982

UL 749

AHAM HS-1

AHAM DW-1 (revision

of HS-1)

CCEE 10

IEC 335-5

ASSE 1006

IEC $335-5$

AHAM DA-8

CUL 73

IP 1971
IP 1972
IP 1972
IP 1972

1970

1971

1967

1968

1969

1960

1971

1968

1971

1967

1969

1972

ANSI C33.11

(1972)

1965

IP 1972

IP 1972

1971

1968

IP 1972

1960

1971

1971

1971

1964

1972

(1971)

ANSI B97.1

(1970)

(1970)

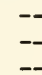

ANSI C33.69

(1971)

$\begin{array}{ll}-- & \text { S } \\ -- & \text { PS } \\ -- & \text { P } \\ -- & \text { S }\end{array}$

ANSI B9.1 S

$\begin{array}{ccl}-- & P & \text { AHAM } \\ -- & \mathrm{P} & -- \\ \text { ANSI } \mathrm{B} 38.1 & \mathrm{O}(\mathrm{TM}) & --\end{array}$

$\begin{array}{ccl}\text { ANSI B97.1 } & \text { S } & \text { UL } \\ (1971) & & \\ -- & \text { P } & \text { AHAM } \\ \text { ANSI B38.1 } & \text { O(TM) } & --\end{array}$

$\mathrm{S}$

CEE

AGA

$-$

$-$

UL

$-$

UT

UL

AHAM

$--$

CEE

ASSE

--

$\begin{array}{ll}\text { ANSI } \overline{\mathrm{C}} 33.36 & \mathrm{P} \\ \mathrm{S}\end{array}$

$-$ 
Product Product

Number

Latest

Issue or

zation and Standard Designation

Status of Standard

Standard Accepted by Standard

Certifi-

cation

AHAM FB-1

IP 1972

CUL 982

IP 1972

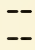

$\overline{U L}$

P0216 Flectric broilers and grills, counter-top ovens

CUL 499

1972

ANSI C33.11

S

(1972)

$\mathrm{CEE} 11$, pt.I and

pt. 2 , sec.M

AHAM DA-3

IEC 335-9

IEC 335-6

0217 Electric coffeemakers and teapots AHAM DA-4

CUL 499

$1964 \& 67$

1969

1972

1972

1968

1972

AHAM CM-1

CCEE 11, pt.1 and pt. 2 , sec. $B$

UL 1082

IP 1972

$1964 \& 66$

IP 1972

CUL 982

AHAM

IP 1972

IP 1972

AHAM DA-9

0219 Electric deep fryers

CUL 499

1964

1972

AHAM

UL 1083

0220 Electric defroster devices

CUL 499

IP 1972

IP 1972

1972

CUL 499

trays

CEE 11, pt.1 and pt.2, sec.M

1972

ANSI C33.11

(1972)

$1964 \& 67$

AHAM DA-9

AHAM FPG-1

CCEE 11, pt.1 and $\mathrm{pt} .2$, sec.M

UL 1083

1964

IP 1972

$1964 \& 67$

IP 1972

ALAM DA-9

AHAM FPG- 1

UL 1083

0224 Electric hot plates

AHAM DA-5

CUL 499

AHAM

CEE 11, pt.1 and pt.2, sec.M

1964

IP 1972

IP 1972
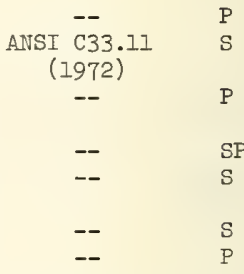

ANSI C33.11

(1972)

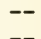

ANSI C33.11

(1972)

1958

1972

IP 1972

1964867

CUL 73

1972

CUL 982

IP 1972

CUL 73

CUL 982

1972

IP 1972

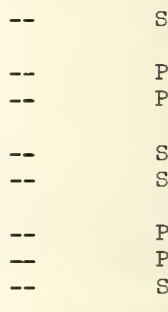

ANSI $\overline{\mathrm{C}} 33.11$

(1972)

CEE

0225 Electric ice cream makers

0226 Electric ice crushers

UL 563

Electric ice makers, separate from refrigerators
ANSI C33.36 (1972)

ANSI C 33.36 (1972)

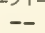

UL

UL

UL

UL

UL 
\begin{tabular}{ll}
$\begin{array}{l}\text { Product } \\
\text { Number }\end{array}$ & $\begin{array}{l}\text { Product } \\
\text { Description }\end{array}$ \\
\hline
\end{tabular}

P0228 Electric Juicers

0229 Electric kettles

0230 Electric meat grinders

0231 Electric mixers

0232 Electric scissors

0233 Electric slicers/choppers

0234 Toasters

0235 Electric waffle irons

0236 Faucet water heaters

0237 Garbage disposers

0238 Irons with dry heat

0239

Steam irons
Latest

Responsible Organi- Issue or zation and Standard Designation

Status of Standard

Standard

Type of

Certifi-

CUL 73

1972
IP 1972
1964866
1972
IP 1972
IP 1972
1972
I972
IP 1972
1960
IP 1972

ANSI C33.36

(1972)

CUL 982

--

Standard

Program

CCEE 11, pt.1 and pt. 2 , sec. $B$ CUL 499

UL 1082

CUL 982

CUL 73

CUL 73
AHAM
CCEE 10
CUL 982

CUL 73

CUI 73

CUL 982

AHAM DA-7

CUL 499

AHAM

CEE 11, pt.1 and pt. 2 , sec.M

IEC 335-9

AHAM DA-3

CUL 499

AHAM

CEE 11, pt.1 and pt.2, sec.M IEC 335-9

UL 430

AHAM FWD-1

ASSE 1008

AHAM DA-1
AHAM
UL 141
ANSI C70.1
IEC $335-3$
IEC 311
CEE 11, pt. 1 and
$\quad$ pt. 2 , sec.G
AHAM DA- 1
AHAM
CEE 11 , pt. 1 and
pt. 2 , sec. $G$ a
UL 141

1972

\begin{tabular}{|c|c|c|}
\hline -- & SP & CEE \\
\hline $\begin{array}{c}\text { ANSI C33.11 } \\
(1972)\end{array}$ & $S$ & UL \\
\hline-- & $S$ & UL \\
\hline-- & $S$ & UL \\
\hline $\begin{array}{l}\text { ANSI C33.36 } \\
(1972)\end{array}$ & $\mathrm{S}$ & UL \\
\hline $\begin{array}{l}\text { ANSI C33.36 } \\
(1972)\end{array}$ & S & UL \\
\hline -- & $P$ & -- \\
\hline-- & S & CEE \\
\hline-- & S & UL \\
\hline
\end{tabular}

ANSI C33.36 S UL (1972)

$1972 \quad$ ANSI C33.36
(1972)

IP 1972

1967

1972

ANSI $\overline{\mathrm{C}} 33.11$

(1972)

IP 1972

1964867

1972

1969

1972

IP 1972

ANSI C33.11

(1972)

$1964 \& 67$

1972
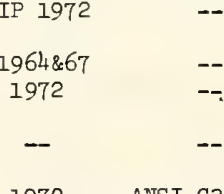

IP 1972

ANSI C33.59

(1970)

1971

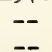

1958

IP 1972

1971

1953

1971

1970

$1964 \& 65$

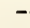

1958

IP 1972

1964865

1954

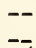

CEस

$-$

--

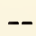

$-$

$--$

$--$

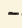

UL

UL

CEE

UL.

L

UL

CEE

UL,

UL

UL

$\overline{U L}$

$-$

CEE

--

$-$

UL

$-$

$-$

UL

$-$

ASSE

$--$

$-$

UL

--

$-$

$-$

CEE

$\begin{array}{lll}-- & P & -- \\ -- & P & --\end{array}$

-- SP CEE

-- S UL




\begin{tabular}{llc} 
Product & Product & Responsible Organi- \\
Number & Description & zation and Standard \\
\hline
\end{tabular}

Latest

Issue or

Status of Standard
P0240 Knife sharpeners, electric

0241 Rotisseries

0242 Ellectric immerson heaters 0243 Ranges/stoves, not otherwise
specified

0244 Ovens, not otherwise specified

CUL 499 specified

0246 Freezers, not otherwise ra

0247 Irons, not otherwise specified

0248 Electric self-cleaning ovens, separate

0249 Gas self-cleaning ovens, separate

0250 Range and oven accessories,
including racks, broiler pans,
etc.

0251 Microwave ovens with ranges

0252 Garbage/trash compactors

0253 Electric range with self-cleaning oven

0254 Gas range with self-cleaning oven

0255 Clothes steamers, personal use

0256 Range/stove hoods and exhaust fans
UL 858
UL 858
AHAM (electric ceramic sooking top)

IP 1972

1972

1969

$C \mathrm{ABYC}$

UL 250

1970

1971

UL 250

ANSI $221.1 .2 \mathrm{~b}$

ANSI Z21.1.1b

1969

1969

AHAM

IP 1972

ANSI C33.36 S

(1972)

UL

UL

UL

$$
\text { (1972) }
$$

ANSI C33.11

S

(1972)

UL

$1964 \& 68$

-- P

ANSI C33.53 S

(1969)

UL

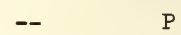

AHAM

ANSI $033.11 \quad \mathrm{~S}$

(1972)

ANSI C33.53 S

(1969)

UL

UL

(1969)
ANSI B97.I
(1971)

UL

AHAM

IP 1972

1971 ANSI B97.1 S

(1971)

UL

S

U

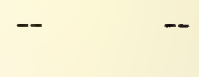

$\mathrm{P}$

AHAM
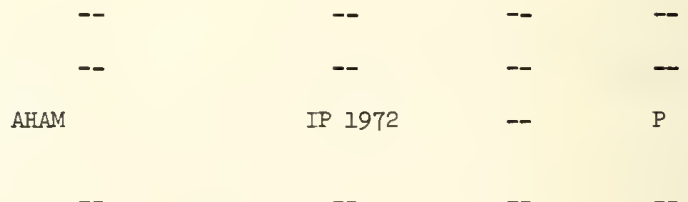

SP

AGA

AGA 
SPACE HEATING, COOLING AND VENTILATING APPLIANCES

P0301 Electric air conditioners

030

Gas air conditioners

0303 Fans, water cooled

0304 Humidifiers

0305 Vaporizers, personel use

0306 Dehumidifiers

$\begin{array}{ll}0307 & \text { Ionizers } \\ 0308 & \text { Boilers }\end{array}$

0309 Coal furnaces

0310 Gas furnaces

$03110 i 1$ furnaces

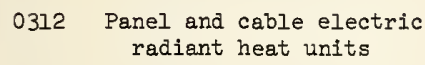

0313 Electric space heaters

UL 484
AHAM CN-1
ARI 210
UL 465

AHAM RAC-1

AHAM RAC-2SR

ARI 310

ISO R859

ARI 443

ANSI Z21.40.2

ANSI Z21.40.1

ARI 620

ARI 610

AHAM HU-1

UL 998

ARI 630

CUL 499

UL 474

AHAM DH-1

ANSI Z21.13

ANSI 221.52

UL 726

UL 834

MCAA *1

USC CS115

ANSI Z21.49

ANSI Z21. 44

ANSI Z21.47

UL 729

UL 727

ANSI Z91.1

AHAM DA-6

AHAM DA-6

UL 573

NEMA HE2

UL 875

UL 1042

UL 1025

$\mathrm{CEE}$ 11, pt.l and pt.2, sec.F

CARI 310

\begin{tabular}{|c|c|c|c|}
\hline 1965 & $\begin{array}{c}\text { ANSI C33.14 } \\
(1971)\end{array}$ & $\mathrm{S}$ & UL \\
\hline 1967 & - & $\mathrm{P}$ & AHAM \\
\hline $\begin{array}{c}1966 \\
\text { (revision }\end{array}$ & IP) & $\mathrm{SP}$ & ARI \\
\hline 1971 & $\begin{array}{c}\text { ANSI B144.1 } \\
(1972)\end{array}$ & $\mathrm{S}$ & UL \\
\hline 1972 & -- & PS & AHAM \\
\hline 1972 & -- & 0 & AHAM \\
\hline 1970 & -- & PS & ARI \\
\hline 1968 & -- & $O(T M)$ & -- \\
\hline 1970 & -- & 0 & ARI \\
\hline 1969 & -- & $\mathrm{S}$ & AGA \\
\hline 1969 & -- & $\mathrm{S}$ & $\mathrm{AGA}$ \\
\hline-- & -- & -- & -- \\
\hline 1968 & - & PO & ARI \\
\hline 1970 & -- & $\mathrm{P}$ & ARI \\
\hline 1971 & -- & $\mathrm{P}$ & AHAM \\
\hline IP 1971 & -- & $\mathrm{S}$ & UL \\
\hline 1972 & -- & 0 & -- \\
\hline 1972 & $\begin{array}{l}\text { ANSI C33.11 } \\
(1972)\end{array}$ & $\mathrm{S}$ & UL \\
\hline 1969 & - & S & UL \\
\hline 1970 & $\begin{array}{l}\text { ANSI B149.1 } \\
(1972)\end{array}$ & $\mathrm{P}$ & AHAM \\
\hline-- & -- & -- & -- \\
\hline 1967 & - & $\mathrm{SP}$ & AGA \\
\hline 1967 & -- & $\mathrm{SP}$ & AGA \\
\hline 1969 & -- & $\mathrm{S}$ & UL \\
\hline IP 1972 & -- & $\mathrm{S}$ & UL \\
\hline $1971-72$ & -- & 0 & -- \\
\hline 1960 & -- & 0 & -- \\
\hline-- & -- & -- & -- \\
\hline 1967 & $-\cdot$. & $\mathrm{SP}$ & AGA \\
\hline 1971 & -- & $\mathrm{SP}$ & AGA \\
\hline 1971 & -- & SP & AGA \\
\hline 1970 & -- & $\mathrm{S}$ & UL \\
\hline 1972 & $\begin{array}{c}\text { ANSI Z96.1 } \\
(1970)\end{array}$ & $\mathrm{S}$ & UL \\
\hline 1972 & -- & $\mathrm{P}$ & -- \\
\hline 1961 & - & $\mathrm{SP}$ & -- \\
\hline 1961 & -- & $\mathrm{SP}$ & - \\
\hline 1968 & $\begin{array}{l}\text { ANSI C33.12 } \\
(1972)\end{array}$ & $\mathrm{S}$ & UL \\
\hline 1969 & - & SP & -- \\
\hline 1970 & -- & $\mathrm{S}$ & UL \\
\hline 1972 & -- & $\mathrm{S}$ & UL \\
\hline IP 1972 & -- & $S$ & UL \\
\hline $1964 \& 68$ & - & $\mathrm{SP}$ & $\mathrm{CEE}$ \\
\hline 1970 & -- & PS & -- \\
\hline
\end{tabular}


Latest

Responsible Organi- Issue or

zation and Standard Status of Designation Standara

Standard

Product Product

Number Description

P0314 Gas space heaters, attached

0315 Kerosene space heaters, attached

0316 Fireplaces, factory built, wood burning

0317 Chimneys, factory built

0318 Electric furnaces

0319 other heating systems, including heat pumps

0320 Radiators, home

0321 Air conditioners, not otherwise specified

\section{ARI 230 \\ $\subset \mathrm{ABYC}$ \\ UL 465}

ASHRAE 79

UL 737

UL 127

UL 103

NFPA 211

UL 959

UL 573

ARI 280

ARI 240

$\subset \mathrm{ABYC}$

UL 573

ARI 280

USC RI74
ANSI $\mathrm{Z} 21.11 .1$
ANSI $\mathrm{Z} 21.11 .2$
ANSI $\mathrm{Z} 21.16$

NFPA 211

ANSI Z21.53

UL 731

UL 730

UL 733

UL 875

UL 1042

ANSI Z21.55

UL 896

AHAM DA-5 specified

0325 Gas (IP) heating stoves

0326 Floor furnaces, not otherwise specified

0327 Floor furnaces, coal

$\begin{array}{llll}1971 & -- & \text { S } & \text { AGA } \\ 1971 & -- & \text { SP } & \text { AGA } \\ 1971 & -- & \text { SP } & \text { AGA }\end{array}$

1971

1971

1971

1971

1971

1971

1968

1968

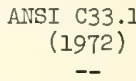

S

$\mathrm{SP}$

ARI ( IP)

ANSI Z181.1 S

(1970)

SI Al63.

(1971)

(1971)

ANSI Al31.I S

(1971)

UL

UL

AGA

ARI

UL

1968

ANSI $\bar{c} 33.12$

(1972)

1972

1965

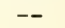

ARI ( IP)

1962

(revision IP)

1970

1971

A

ANSI BI 44.1

(1972)

IP 1972

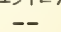

$\overline{U L}$

(TM)

1955

1955

1955

1970

1972

1971

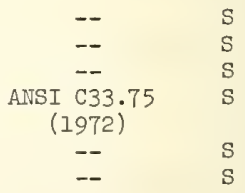

UL

UL

UL

UL

UL

AGA

UL

UL 


\begin{tabular}{|c|c|c|c|c|c|c|}
\hline $\begin{array}{l}\text { Product } \\
\text { Number }\end{array}$ & $\begin{array}{l}\text { Product } \\
\text { Description }\end{array}$ & $\begin{array}{c}\text { Responsible Organi- } \\
\text { zation and Standard } \\
\text { Designation }\end{array}$ & $\begin{array}{l}\text { Latest } \\
\text { Issue or } \\
\text { Status of } \\
\text { Standard } \\
\end{array}$ & $\begin{array}{l}\text { Standard } \\
\text { Accepted by }\end{array}$ & $\begin{array}{l}\text { Type of } \\
\text { Standard }\end{array}$ & $\begin{array}{l}\text { Certifi- } \\
\text { cation } \\
\text { Program }\end{array}$ \\
\hline P0328 & Floor furnaces, gas & $\begin{array}{l}\text { ANSSI. } 221.48 \\
\text { ANSI } 221.47\end{array}$ & $\begin{array}{l}1967 \\
1971\end{array}$ & $\begin{array}{l}-- \\
--\end{array}$ & $\begin{array}{l}\mathrm{SP} \\
\mathrm{SP}\end{array}$ & AGA \\
\hline 0329 & Floor furnaces, oil & $\begin{array}{l}\text { UL } 729 \\
\text { UL } 727\end{array}$ & $\begin{array}{l}1970 \\
1971\end{array}$ & $\begin{array}{l}\text { ANSI } \overline{z 96.1} \\
(1970)\end{array}$ & $\begin{array}{l}S \\
S\end{array}$ & $\begin{array}{l}\text { UL } \\
\text { UL }\end{array}$ \\
\hline 0330 & Gas heating stoves & -- & -- & -- & -- & -- \\
\hline 0331 & Kerosene heating stoves & -- & -- & -- & -- & -- \\
\hline 0332 & Wood stoves & -- & -- & -- & -- & -- \\
\hline 0333 & Coal stoves & -- & -- & -- & - & -- \\
\hline 0334 & $\begin{array}{l}\text { Fireplaces, factory built, gas } \\
\text { burning }\end{array}$ & -- & -- & -- & -- & - \\
\hline 0335 & Floor furnaces, electric & UL 573 & 1968 & -- & s & UL \\
\hline \multirow[t]{8}{*}{0337} & Hot water pipes & ASTM AI20 & 1972 & $\begin{array}{l}\text { ANSI B125.2 } \\
(1970)\end{array}$ & 0 & -- \\
\hline & & ASTM D2241 & 1969 & $\begin{array}{l}\text { ANSI B72:2 } \\
\quad(1967)\end{array}$ & 0 & -- \\
\hline & & and USC PS22 & 1970 & -- & 0 & -- \\
\hline & & ASTM B88 & 1971 & ANSI H23.1 & 0 & -- \\
\hline & & ASTM A72 & 1968 & $\begin{array}{l}\text { ANSI B36.2 } \\
(1969)\end{array}$ & 0 & -- \\
\hline & & ASTM B302 & 1970 & $\begin{array}{l}\text { ANSI H26.2 } \\
(1967)\end{array}$ & 0 & -- \\
\hline & & ASTM B 43 & 1970 & $\begin{array}{l}\text { ANSI H27.1 } \\
(1967)^{1}\end{array}$ & 0 & -- \\
\hline & & ASTM B42 & 1971 & $\begin{array}{l}\text { ANSI H26.1 } \\
(1967)\end{array}$ & 0 & - \\
\hline \multirow[t]{2}{*}{0338} & Steam pipes & ASTM A120 & 1972 & $\begin{array}{l}\text { ANSI B125.2 } \\
(1970)\end{array}$ & 0 & - \\
\hline & & ASTM AT2 & 1968 & $\begin{array}{l}\text { ANSI B36.2 } \\
(1969)\end{array}$ & 0 & -- \\
\hline 0339 & $\begin{array}{l}\text { Ductwork for heating or cooling } \\
\text { systems }\end{array}$ & $\begin{array}{l}\text { UL } 181 \\
\text { CSMACNA *4 } \\
\text { CSMACNA *6 } \\
\text { CSMACNA ( code) }\end{array}$ & $\begin{array}{l}1970 \\
1969 \\
1969 \\
1967\end{array}$ & $\begin{array}{l}-- \\
-- \\
--\end{array}$ & $\begin{array}{l}\text { S } \\
0 \\
O P \\
0\end{array}$ & $\begin{array}{l}\text { UL } \\
-- \\
-- \\
--\end{array}$ \\
\hline 0340 & $\begin{array}{l}\text { Thermostats for any heating/ } \\
\text { cooling system }\end{array}$ & NEMA DC3 & 1972 & - & 0 & -- \\
\hline \multirow[t]{3}{*}{0341} & $\begin{array}{l}\text { Fans, permanently installed, } \\
\text { including attic fans }\end{array}$ & $\begin{array}{l}\text { IEC } 176 \\
\text { IEC } 342 \\
\text { UL } 507\end{array}$ & $\begin{array}{l}1966 \\
1971 \\
1969\end{array}$ & 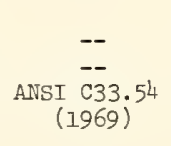 & $\begin{array}{l}P \\
S \\
S\end{array}$ & $\overline{--}$ \\
\hline & & AMCA 210 & 1967 & -- & 0 & AMCA \\
\hline & HOUSEWARES & & & & & \\
\hline 0401 & Can openers, unpowered & -- & -- & -- & -- & -+ \\
\hline 0402 & $\begin{array}{l}\text { Chafing dishes and fondue pots } \\
\text { with open flame burners }\end{array}$ & - & - & - & - & - \\
\hline 0403 & Table stoves, open flame & ANSI Z21.9 & 1967 & -- & s & AGA \\
\hline
\end{tabular}


POLOL Non-electric coffee grinders

0405 Coffeemakers and teapots, unpowered

0406 Pots and pans, including lids

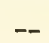

1970

1947

1970

1970

1971

1971

1970

1970

1970

1970

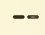

UL 136

CCEE 11, pt.1 and pt.2, sec.B

$1964 \& 66$

IP 1971

CUL 982

1970

1971

1970

1971

1970

1970

1970

1971

1970

1970

1970

1970

$1971 \mathrm{~T}$

ASTM C368

USC PS24

ASTM C424

ASTM C554

ASTM C327

ASTM C408

ASTM C676
0

O(TM)

$O$ (TM)

$O(T M)$

$O$ (TM)

$O(T M)$

$O(T M)$

$O$ (TM)

$\mathrm{O}(\mathrm{TM})$

0413 Waste containers, trash can/ basket, etc.

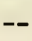

--
--
--
--
--
--
--
--
--
--
- 
P0421 Knife sharpeners, manual

0422 Bottle openers

0423 Butane candles

0424 Drinking glasses, glass

0425 Drinking glasses, non-glass, including plastic, paper cups and pottery mugs

0426 Ovenware, including glass and pottery

0427 Ice picks

0428 other kitchen gadgets, including mixing and measuring spoons/cups, strainers, spatulas, rolling pins, cake decorators, etc.

0429 Meat skewers

0430 Ice cream makers, manual

0431 Toothpicks and hors d'oeuvre picks, wood, plastic and metal

0432 Trays, including folding TV trays

0433 Candle holders, candlesticks

0434 Door stops

0435 Kitchen mixing bowls, canisters and similar containers

0436 Potholders, oven mits, hot pads

0437 Tableware accessories, including salt/pepper shakers, sugar bowl, cream pitchers, etc.

0438 Laundry baskets

0439 Bar accessories, swizzle sticks, seltzer bottles, manual ice crackers, etc.

0440 Thermometers, atmospheric

0441 Thermometers, cooking

HOME COMMUNICATIONS, ENTERTAINMENT, \& TOBBY EQUTPMENT

0501 TV sets, not otherwise specified

0502 Radios, all models

UL 492

1968

ANSI C33.55

(1969)

0503 Sound recording and reproducing equipment (tape recorders and players)

CUL 492

1968

ANSI C33.55

(1969)

IEEE 193

1971 ANSI S4.3

(1972) 
EIA REC133

IGEE 347

IEC $94 \mathrm{~A}$

1949

1972

1972

Accepted by

thandara Prog

Standard Program

P0504 Musical instruments, non-electric

0505 Movie cameras

UL 122

IP 1972

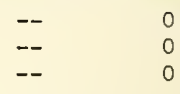

0506 other photographic equipment and accessories, including editors and splicers

ANSI PH3.49

UL 122

ANSI PH3.15

ISO 1229

IP 1972

1972

1972

0507 Movie projectors

CCEE 10

UL 122

1960

IP 1972

ANSI PH3.27

ANSI PH3.28

UL 122

0509 Intercommunication Devices

CUL 492

IEEE 184

IEEE 269

1971

1970

ANSI X4.12

(1970)

0512 TV and radio antennas, indoor

EIA RECI 41

UL. 452

1954

1972

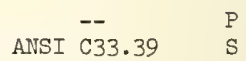

(1972)

USC PS30

USC CS98

USC RI92

USC CS130

1970

1962

1963

1960

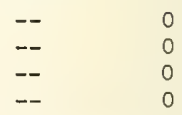

0514 Clay, pottery and ceramic supplies and equipment (not including tableware)

0515 Printing presses (for home use)

0516 TV sets, console, black and white, including table models UL 492

NCRPM *1

1968

ANSI C 33.55

(Interim)

1968

0517 TV sets, portable, color

UL 492

1968

ANSI C33.55

NCRPM * 1

(Interim) 1968

UL 492

1968

ANSI C33.55

NCRPM *1
UL

UL.

UL

$-$

CEE

UL

UI

UL

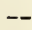

UL

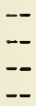

(1969)

$-$

S

(1969)

S

(1969)

UI.

S

$-$

UT.

UL
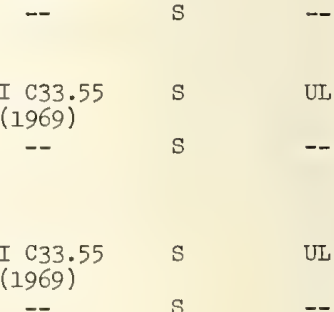

S
(Interim) 1968 
$0520 \mathrm{Hi} F i$ and stereo components and equipment, including amplifiers, remote speakers

CIEC 65

IEC 268-3

IHF A201

EIA SE103

CUL 492

1965
1969
1966
1949

0521 Record players, self-contained units

1968

ANSI C33.55

(1969)

0522 Electric musical instruments

0523 Cameras, still picture and slide

CUL 492

ISO R5I6

ISO R517

ISO R519

1968

1966

1966

1966

0524 Flash attachments for cameras, flash bulbs

0525 Movie light bar

$0526 \mathrm{TV}$ and radio antennas, outdoor

0527 Movie screens and stands

0528 TV sets, console, color, including table models

UL 492

1968

ANSI C33.55

(1969)

ANSI C33.55

(1969)

$--$

$1965)$
--
--
--

$--$

$-$

$\pi$

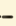

$\pi$

$-$

$-$

0529 Telescopes, binoculars, microscopes and other optical equipment

0530 Phonograph records

RIAA Bul. $\mathrm{E}_{4}$

1966

RIAA BuI.E3

EIA RS2lIC

1963

1971

RIAA Bul.E5

RIAA *1

1969

1966

IEC 94 and $94 \mathrm{~A}$

1968

0532 Glass bottle cutting equipment/ kits

0533 Lapidary equipment and tools including rock tumblers, polishers, etc.

$0534 \mathrm{Film}$ for all cameras
ANSI PHI. 14

ANSI PHI. 21

ANSI PHI. 25

ANSI PHI. 26

ANSI PHI. 29

ANSI PHI. 31

ANSI PHI. 37

ANSI PH22. 5
1971
1972
1969
1969
1971
1971
1969
1969

ISO R1007

(1969)

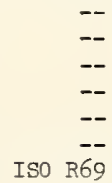

(1958)
UL

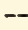

$--$

$-$

$-$

$-$

$-$

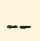

$-$

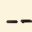

$-$

$-$

$-$

$--$

$-$ 


$\begin{array}{ll}\text { ANSI PH22.12 } & 1969 \\ \text { ANSI PH22.109 } & 1969 \\ \text { ANSI PH22.110 } & 1969 \\ \text { ANSI PH4.33 } & 1969 \\ \text { ISO R421 } & 1965 \\ \text { ISO R686 } & 1968 \\ \text { ISO R732 } & 1971 \\ \text { ISO RI007 } & 1969 \\ \text { ANSI PH3.39 } & 1971 \\ \text { UL } 105 & 1972\end{array}$

$\begin{array}{cc}\text { ISO R69 } & 0 \\ (1958) & \\ -- & 0 \\ -- & 0 \\ -- & 0 \\ -- & 0 \\ -- & 0 \\ -- & 0 \\ -- & 0 \\ \text { ISO RI754 } & 0 \\ (1971) & \\ -- & \text { S }\end{array}$

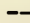

$(-1069$

1965

1968

1971

1972

1962

P0601 Beds, including springs, frames

USC R2

NEMA WD3
1963

CEE 7

1959

CEE 14

CEE 16

CEE 24

UL 514

UL 198.3

UL 198

UL 869

UL 511

ANSI C73 series

UL 486

UL 20

UL 414

CNEPA 70 and $70 \mathrm{~A}$

UL 50

UL 498

NEWMA WD5

1970

1962

1972

1969

1970

1971

1951

1971

$1961-66$

1969

1972

1957

1969

1970

1962

1972

1969

1967

NEMA MGI

ASTM A120

1972

ANSI B125.2

(1970)

1971

ASTM D2513

ASTM A72

1968

1967

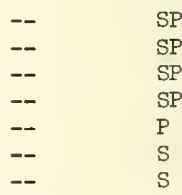

ANSI C33.42

(1971)

ANSI C33.21

(1971)

ANSI C 33.5

(1969)

ANSI C33.40 S

(1972)

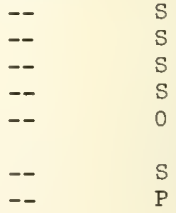

ISO R559
$--$

ANSI B36.2

(1969)

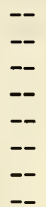

UI
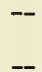

CEE

CEF

CEE

$\mathrm{CEF}$

$--$

UL

UI

UL

UL

UL

UL

UI

UI

UL

--

$-$
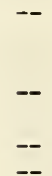

$-$ 


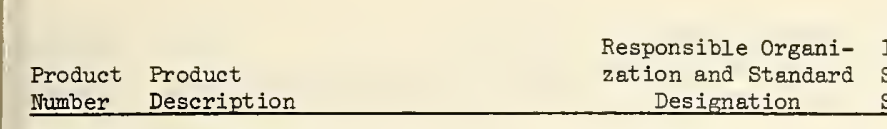

Latest

\section{P0608 Plumbing pipes}

ASTM B88
NSF 14
IAPMO PS2-66
ASTM B 42
ISO R161
ASTM D2239

and USC PSIl

ASTM B306

ASTM D2447

and USC PSI2

ASTM D2104

and USC PSIO

ASTM D1527

and USC PSI8

ASTM D2282

and USC PS19

ASTM D1785

and USC PS2I

ASTM D2241

and USC PS22

ASTM D2672

ASTM D2665

and IAPMO PS27

ASTM D266I

and IAPMO PSIT

IAPMO PS28

IAPMO PS29

and ASTM B543

ASTM Al20

ASTM B 43

ASTM A72

ASTM A74

ASTM A312

ASTM A651

ASTM A53

ASTM A268

ASTM B135

ASTM B75

ASTM B251

IAPMO PS24

ISO R559

ASTM B315

CANSI 297.1

CGTA
Issue or

Status of Standard Type of cation Standard Accepted by Standard Program

1971 ANST H23.1 0

1970

1966

1971

1960

1971

1969

1971

1971

1969

1971

1969

1971

1969

1971

1969

1968

1970

1969

1970

1968

1968

1969

1971

1971

1969

1969

$1970 \mathrm{~T}$

1972

1970

1968

1969

1971

1971

1971

1971

1971

1968

1971

1971

1967

1970

ANSI B72.1

(1970)

(1970)

ANSI

(1970)

$--$

--

$-$

--

ANSI B72.3

(1970)

ANSI $\overline{\text { BT2 }}$.7

(1971)

ANSI $\overline{\text { BT2. }}$.

(1967)

ANSI B72.20

(1971)

ANSI K65.56

(I971)

--

$-$

ANSI$$
-
$$

$$
\text { (1970) }
$$

ANSI H27.I

(1967)

ANSI B36.2

(1969)

ANSI AlI2.5.I 0

(1971)

ANSI B125.16

(1970)

ANSI B125.1

(1970)

ANSI B125.14

(1970)

ANSI H36.1

(1969)

ANSI H23. 3

(1970)

ANSI H23.4

(1970)

$-$

1972

1969
$-$

$-$

$--$

-

NSF

IAPMO

$-$

$-$

$-$

$-$

$-$

$-$

$-$

$-$

$-$

$-$

$-$

$-$

$-$

$-$

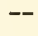

$-$

-

IAPMO

-

IAPMO

IAPMO

IAPMO

$-$

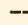

$--$
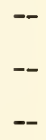

$-$
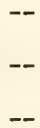

$-$

--

IAPMO

--

$\begin{array}{ll}-- & \text { PS } \\ -- & \text { SP }\end{array}$

- 
P0610 Bathtub and shower enclosures of materials other than glass

ANSI ZI24.I
ANSI Z124.2
IAPMO PSII
GA ${ }^{\circ} 17$

1967

1967

1971

1970

06II Bathtub and shower stmuctures other than doors and panels, including the tub, walls, handgrips, etc.

0612 Runners and throw rugs, door mat

ANSI Z124.1

1967

ANSI $\mathrm{Z} 124.2$

CUSC PS5

CUSC CST7

IAPMO PSII

CANSI L22.30.2

1967

1966

1963

1971

1968

0613 Carpeting, including outdoor, but excluding runners

ISO R2095

ASTM D2646

1971

ASTM D2859

ASTM D1116

ASTM D2401

ASTM D1335

ASTM D 418

ISO 2549

Iso 2550

ISO 255 I

ANSI I22.30.8

ANSI I22.30.7

ABFIO J-I

ABFLO J-2

ABFLO J-3

1969

1970T

1969

1967

1967

1968

1972

1972

1972

1968

1968

1968

1968

1968

0616 Blankets, except electric and baby blankets

ANSI L22.30.4

1968

ANSI L22.30.6

1968

ANSI IK2.30.9

ANSI I.22.30.15

1968

1968

0618 Stepladders

ANSI AI4.1

1968

ANSI Al 4.2

MIMA *I

UL 184

UL 112

1972

1968

1971

1969

ANSI AI4.I

MLMA *I

1968

1968

UL 184

UL 112

1971

1969

0620 Stepstools

062I Electric cords, extension cords, and replacement wire
CEE 2

CEE 13
$1962-68$ 1962
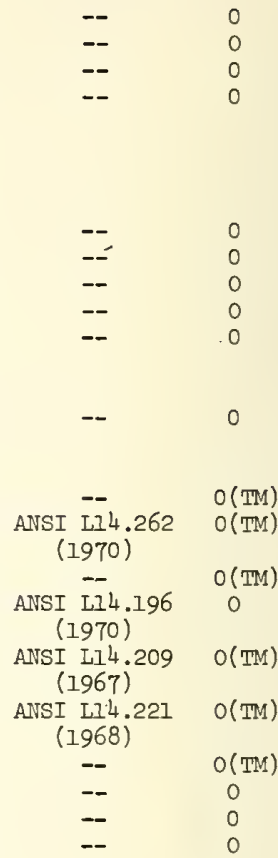

$--$

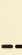

$-$
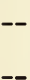

$-$

$-$

$-$

-

$-$

--

-

$-$

$-$

$-$

UL

$-$

UL

UL,

$-$

CEE

CEE 
Product Product

Number
Description

Responsible Organi- Issue or

zation and Standard Designation

Status of

Certifi-

Standard Accepted by Standard Program

UL 115

UL 83

UL 817

UL 133

UL 44

UL 62

IEC 227A

\begin{abstract}
P0622 Gas meters
0623 Meters for LP gas

0624 Electric meters
\end{abstract}

0625 Gas lamps, installed

0626 Electric table lamps and floor lamps, desk lamps

0627 Light bulbs
0628 Electric Light Fixtures,
attached, chandeliers, hanging
lamps

0629 Electric clocks

UL 57

CGrA

\section{AHAM \\ CEE 10, pt.1 and \\ pt.2, sec.E}

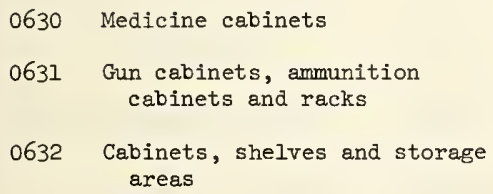

0630 Medicine cabinets

0631 Gun cabinets, ammunition cabinets and racks

0632 Cabinets, shelves and storage areas
0633 Sump pumps
0634 Furniture, not otherwise specified

0635 Fabrics, not otherwise specified

$\begin{array}{cccc}1970 & \text { ANSI C33.66 } & \text { S } & \text { UL } \\ & (1970) \\ 1963 & \text { ANSI C33.80 } & \text { S } & \text { UL } \\ & (1971) \\ 1962 & \text { ANSI C33.3 } & \text { S } & \text { UL } \\ 1970 & (1971) & & \text { UL } \\ 1968 & - \text { ANSI C33.6 } & \text { S } & \text { UL } \\ 1971 & (1969) & & \text { UL } \\ & \text { ANSI C33.1 } & \text { S } & \\ 1971 & (1971) & \text { S } & --\end{array}$

1968

1970

1969

$1966 \& 67$

1960

1964

1970

1949-71

1972

1969

ANSI C33.71
UL 826

CCEE 10

1968

IP 1972

$1964 \& 67$

1960

ANSI A]61.1

UL 65

1970

1971

NAPF $\quad C-1-70$

1970

(1971)

1971

SPMA *1

1965

1960

1969

ANSI C33.47 S

(1968)

-- P

-- $\quad$ SP

$--\quad S$

USC CS273

CANSI L22 series

NAFM *I
CEE

UL

UL

UL

UI

UL

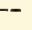

UL

UL

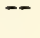

$-$

$-$

UL

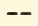

UL GTA

UL

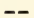

$-$

UL

-- P --


Product Product

Number Description

P0636 Ladders or stepstools, not otherwise specified
ANSI A]4.1

0637 Meters, not otherwise specified

(for household utilities only) AWWA C700

0638 Window shades and venetian blinds, indoor shutters

0639 Flashlights and electric lanterns UL 153

0640 Mirrors and mirror glass

0642 Bedding, including mattresses, mattress covers and pads

0643 Beach chairs, folding furniture, multiple use, patio furniture

0644 Chairs, not upholstered or not otherwise specified

0645 Tables, cocktail and coffee tables

0646 End tables

0647 Couches, sofas, sofa beds, convertible sofas, etc., including cushions

0648 Sinks

0649 Toilets

0650 Slip covers

0651 Table linens

0652 Pictures and picture frames, wall decorative items

0653 Artificial flowers and plants

0654 Knick-knacks, vases, statues, urns
MLMA *1

UL 184

UL 112

AWWA C706

1968

1971

1969

1971

1972

SAF J596

1970

1960

USC R2

ANSI L27.I

1962

AHAM DA-2

UL 964

ABFLO J-4

ASTM D2971

1971

1967

1968

1968

1971

CUSC CS2O

CUSC PS5

CUSC CS77

IAPMO PSI8

IAPMO TSCl6

CUSC CS243

CUSC Cะ20

IAPMO TSC-I

IAPMO TSC-12

ANSI L22.30.10

1963

1966

1963

1971

$1967 \mathrm{~T}$

1962

1963

1967

1965

1968

ANSI L22.30.11

1968

ANSI C33.52

(1969)

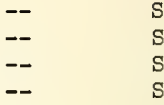

--
UL
UL

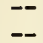

--

UL.

--

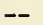

$-$

$-$

UL
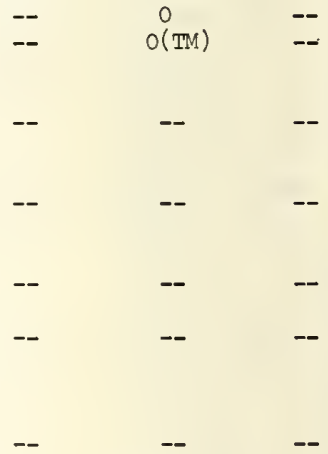

$-$

$\begin{array}{ll}-- & 0 \\ -- & 0 \\ -- & 0 \\ -- & 0 \\ -- & 0 \\ -- & 0\end{array}$

$-$

$-$

$-$

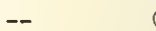

0 
P0655 Drapery and curtain rods, all kinds

0657 Towel racks and bars

0658 Bars and bar stools

0659 Inflatable furniture, all kinds

0660 Clocks, other than electric

0661 Bunk beds, not including bedding

0662 Waterbeds/water pillows

0663 Fireplace equipment, including andirons, screens, tools, grates, non-wood logs

0664 Freestanding book shelves, room dividers/screens

0665 Footstools/ottomans, hassocks

0666 Bathtowels/cloths, beach towels, dishtowels/cloths

CANSI L22

1968

0667 Bedspreads, "throws," Comforters CANSI L22

0668 Books, magazines, albums

USC CS49

USC CS50

USC PS9

\section{(under revision)}

1934

1934

1968

0669 Clothespins and similar fasteners

0670 Recliner chairs

0671 Rocking chairs

0672 Laundry hampers

0673 Drapery hooks/rings, shower curtain hooks

0674 Glass tables - all kinds, indoors and outdoors

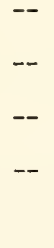

HOME AIARM, ESCAPE, AND PROTECTION DEVICES

\section{1}

Fire extinguishers

0702
UL 711

UL 154

UL 7

UL 626

UL 8

UL 715

UL 299

NFPA $10 \mathrm{~A}$

UL 521

UL 464
1969

1971

1969

1971

1970

1970

1971

1970

1970

1968

$\begin{array}{cc}-- & \text { S } \\ \text { ANSI Z171.1 } & \text { S } \\ (1969) & \\ -- & \text { S } \\ -- & \text { S } \\ -- & \text { S } \\ -- & \text { S } \\ -- & \text { S }\end{array}$

ANSI Z220.1 S

(1971)

AINSI C 33.43 S

(1968)
GTA
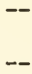

$-$

$-$

$-$

$-$

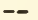

GTA

UL.

UL

UL

UL

UL

UL

UL

$--$

UL

UI 
Latest

UL 168

NEMA SB2

NFPA 74

UL 167

CNFPA 101

1971
1964
1967
IP 1971

ANSI Al31.4

(1971)

IP 1971

P0703 Fire escape devices,
including chain ladders

0704 Burglar alarms

UL 464

UL 639

UL 1023

UL 904

ASTM

0705 Circuit interrupters and circuit breakers

ANSI C37.7

CEE 18

UL 877

UL 943

ANSI C37.14

ANSI $\mathrm{C} 37.15$

NEMA SG3

CEE 19

0706 Lightning arresters, rods, and grounding devices

NFPA 78

ANSI $\mathrm{C} 62.2$

UL 467

IEC $99-1$

UL 452

IEEE 28

NEMA IAI

UL 943

IEEE 32

0707 Locks, padlocks, and chain locks

\section{BHMA 501}

BHMA 601

ASTM

UL 768

UL 887
1970

1968

1970

1972

1972

IP 1972

1971
1959
IP 1971
IP 1971
1969
1967
1971
1959

1968

1969

1967

1970

1972

1972

1968

IP 1972

1972

1971

1970

IP 1972

1972

1972
ANSI A156.2

(IP 1972)

ANSI SE4.2

(1972)

ANSI SE4.3

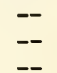

ANSI A9.1

(1971)

ANSI C33.43

(1968)

$\begin{array}{ll}-- & S \\ -- & S \\ -- & S \\ -- & --\end{array}$

UL.

UL

UL

UL

--
UL

UL

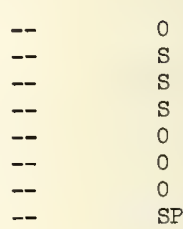

--
UL
UL
--
--
--
$C E E$

$\begin{array}{cc}\begin{array}{c}\text { ANSI C5.1 } \\ (1969)\end{array} & \text { S } \\ -- & \text { S } \\ \text { ANSI C33.8 } & \text { S } \\ (1967) & \\ -- & 0 \\ \text { ANSI C33.39 } & \text { S } \\ (1967) & \\ \text { ANSI C62.1 } & \text { S } \\ (1967) & \\ -- & \text { P } \\ -- & \text { S } \\ -- & \text { S }\end{array}$

(1972)

HOME WORKSHOP APPARATUS, TOOLS AND ATTACHMENTTS

0801 Power saws, not otherwise specified

CUL 45

CUL 987

0802 Power drills

CUL 45

CCEE 20

CUL 987

0803 Power sanders
CUL 45

CCEE 20

CUL 987
1972

1971

1972

1960

1971

1972

1960

1971

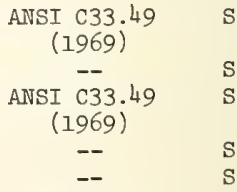

ANSI C33.49

(1969)
UL

UL

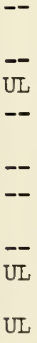

UL 
$\begin{array}{llc}\text { Product Froduct } & \text { Responsible Organi- } \\ \text { Number } & \text { Description } & \text { zation and Standard } \\ \text { Designation }\end{array}$ Standard

CUL 45

0805 Power lathes

0806 Power grinders

0807 Power jointers

0808 Power shapers

0809 Other portable and stationary power tools

0811 Torches

0812 Welding equipment, electric

0813 Soldering guns and irons

0814 Hoists, lifts, jacks and heavy duty chains

0815 Test equipment, voltage testers

0816 Battery chargers

0817 Batteries, all kinds

CUI 45

CUI 45

UL 123

UL 551

UL 516
CUL 987

CCEE 20

CUL 987

CUL 987

CUL 987

CCEE 20

CUL 987

ISO R236

ISO RI711

ISO R234

ANSI 249.1

NEMA EW-1

cuL 499

CANSI AI7.I

ANSI B30.I

NEMA RI2

ANSI C18.1

IEC $86-1$

IEC $86-2$

IEC $86-3$

BCI *1. 15

BCI *1.41

BCI *1.48

SAE J537g

SAE J538a

ASTM D639

SAE J 240

0818 Extension work lights and continuous use flood lights

0819

Separate electric motors

NEMA FLI

UL 276
ANSI C33.49 S UL

(1969)

$1972 \quad$ ANsI C33.
(1969)

1971

1972

1960

1971

1971

1971

1972

1960

1971

1961

1970

1961

1963

1972

1967

1971

1972

1971

1952

ANSI C33.49

(1969)

$-$$$
\text { ( }
$$

--
--
--

$-$

ANSI C 33.2

(1971)

ANSI $\overline{\mathrm{C} 87.1}$

(1971)

ANSI C33.11 S UL

(1972)

$\begin{array}{lll}-- & S & - \\ -- & S & --\end{array}$

1966

1940

(revision IP)

$\begin{array}{ll}-- & 0 \\ -- & S\end{array}$

$-$

1969

1962

1963

1965

1970

1970

1970

1971

1968

1972

1971

$\begin{array}{ll}-- & 0 \\ -- & 0 \\ -- & 0 \\ -- & 0 \\ -- & 0 \\ -- & 0 \\ -- & 0 \\ -- & 0 \\ -- & 0 \\ -- & 0(T M) \\ -- & 0\end{array}$

1966

1964

IP 1971

1969

1967

1969
UL

UL

UL

CEE

UI

UT

UL

UL

$-$

UL 
P0820 Internal combusion engines, gasoline engine

0821 Automotive tools and accessories, tire chains, license plates, tire irons

0822 Paint sprayers

CUL 73

0823 Air compressors, separate

0824 Power saws, portable, not circular, electric

0825 Power saws, stationary

ANSI 394.2

0827 Hammers

0828 Screwdrivers

0829 Manual drills

0830 Manual saws
SAE J565b

SAE J915

$\mathrm{SAE} \mathrm{J} 4 \mathrm{C}$

SAE J5830

SAE J638

SAE J78TD

SAE J651b

SAE J902a

SAE J903b

SAE J942a

SAE J80

ISO $\mathrm{R} 303$

UL 904

CCEE 20

CAGI * 1

CCEE 20

CUL 987

ANSI B94.11

CCEE 20

SI B121.

ISO 2336

1969

1965

1965

1970

1960

1966

1967

1967

1968

1971

1957

1963

1970

1967

1960

1963

1960

1971

1967

1971

1960

CCEE 20

ISO 2380

ISO 2351

ANSI B94.II

ANSI B94.12

ISO R235

1960

1972

1972

1967

1968

1961

1970

1972
Accepted by Standard

Program

0831 Welding equipment, fuel powered

0832 Power saws, portable, circular

0833 Workshop compounds/chemicals, soldering compounds, abrasive compounds

0834 Workshop staplers, heavy duty

0835 Tool boxes/tool kits (only the box itself, not the tools)

HOME AND FAMILY MAINTENANCE PRODUCTS

0901 Cleaning agents and compounds (soap, cleanser, laundry detergent)

ANSI C33.36 SP (1972)

UL

CEF

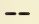

CEE

UL

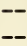

CEE

CEF
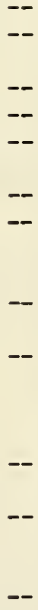
Latest

Responsible Organi- Issue or

Product Product

zation and Standard Status of

Standard

CertifiDesignation

Standard

Accepted by Standard Program

ASTM DIIII

ASTM DIl12

ASTM D799

ASTM D497

ASTM D499

ASTM D455

ASTM D2179

ASTM D533

ASTM D496

ASTM D498

ASTM D690

ASTM D630

ASTM D2957

ASTM D2955

ASTM D534

ASTM D691

ASTM D2958

ASTM D593

ASTM D2815

ASTM D802

CSMA (carpet shempoos)

CSMA BuI.180

CSMA BuI. 41

CSMA Bul.198

ASTM D929

ASTM D2356

CTFA 4

ASTM D2763

ASTM D2762

CSMA Bul.12

A.STM DI 437

CSMA Bul.335

AVATI *6

ASTM D1792

ASTM D2834

ASTM D1436

ASTM D2869

ASTM D1793

ASTM D2047

CSMA Bul. 81
1969

1969

1969

1969

1969

1969

1970

1969

1967

1967

1967

1967

$1971 T$

$1971 \mathrm{~T}$

1967

1967

1971

1969

1972

1970

IP 1971

1960

1959

$1957 \mathrm{~T}$

1970

1972

1965

1970

1970

1971

1971

1971

1964

1966

$1969 \mathrm{~T}$

1971

$1970 T$

1966

1969

1966

ANSI $\overline{\mathrm{K} 60.14}$

$$
\text { (1970) }
$$

ANSI $\mathrm{K} 60.2$

$$
\text { (1970) }
$$

ANSI K60.4

(1970)

ANSI K60.6

(1970)

ANSI $\overline{\mathrm{K}} 60.7$

$$
\text { (1970) }
$$

ANSI K60.I (1969)

ANSI K60.3 (1969)

ANSI K60.8

(1969)

ANSI $\mathrm{K} 60.15$

(1969)

--
ANST $\overline{--}$
$(1969)^{9}$

$\begin{array}{ccc}\text { ANSI K60.13 } & 0 & -- \\ (1970) & 0 & - \\ -- & 0 & - \\ -- & 0(T M) & -\end{array}$

$\begin{array}{ll}-- & 0(\mathrm{TM}) \\ -- & 0(\mathrm{TM}) \\ -- & 0(\mathrm{TM}) \\ -- & 0(\mathrm{TM}) \\ -- & 0 \\ -- & 0 \\ -- & 0\end{array}$

--
--
--
--
--
--

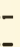

\begin{tabular}{|c|c|c|}
\hline-- & 0 & - \\
\hline-- & 0 & - \\
\hline- & $S$ & - \\
\hline $\begin{array}{c}\text { ANSI } 2123.8 \\
(1966)\end{array}$ & $S(T M)$ & \\
\hline-- & $O(T M)$ & \\
\hline-- & 0 & \\
\hline $\begin{array}{c}\text { ANSI Z123.11 } \\
(1966)\end{array}$ & $O(T M)$ & \\
\hline- & $O(T M)$ & \\
\hline $\begin{array}{c}\text { ANSI Z123.7 } \\
(1966)\end{array}$ & $O(T M)$ & \\
\hline - & $O(T M)$ & \\
\hline $\begin{array}{c}\text { ANSI Z123.12 } \\
(1966)\end{array}$ & $S(T M)$ & \\
\hline- & $S(T M)$ & \\
\hline-- & $O(T M)$ & \\
\hline
\end{tabular}

P0902 Bleaches anded for cosmetic use
intende

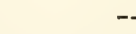




\begin{tabular}{|c|c|c|c|c|c|c|}
\hline & & CSMA Bul. 117 & 1968 & -- & $S(T M)$ & -- \\
\hline & & CCSMA Bul. 245 & 1970 & -- & $S(T M)$ & -- \\
\hline & & CSMA Bul.60 & 1968 & -- & $O(T M)$ & - \\
\hline & & ASTM DI 437 & 1964 & -- & $S(T M)$ & -- \\
\hline P0905 & Polishes, furniture & CSMA Bul. 46 & 1972 & - & 0 & -- \\
\hline 0906 & Fumigants & USC CST2 & 1954 & -- & 0 & -- \\
\hline & & ASTM (pesticides) & IP 1972 & -- & 0 & -- \\
\hline & & CAOAC (chapter 4) & 1965 & -- & 0 & - \\
\hline 0907 & $\begin{array}{l}\text { Paints, varnishes, shellacs, } \\
\text { rust preventatives, etc. }\end{array}$ & ASTM D2571 & 1971 & -- & 0 & -- \\
\hline & & ASTM D2931 & I970T & -- & 0 & - \\
\hline & & ASTM D2932 & 1970T & -- & 0 & -- \\
\hline & & ASTM D360 & 1955 & -- & 0 & -- \\
\hline & & ISO R1515 & 1970 & -- & $S$ & -- \\
\hline & & ANSI Z66.1 & 1972 & -- & $\mathrm{S}$ & -- \\
\hline & & CUL 723 & 1971 & $\begin{array}{r}\text { ANSI A2.5 } \\
(1970)\end{array}$ & $\mathrm{S}(\mathrm{TM})$ & UL \\
\hline & & ASTM D2348 & 1968 & -- & $S(T M)$ & -- \\
\hline & & ASTM D2088 & 1968 & -- & $O(T M)$ & - \\
\hline & & Iso R56 & 1957 & -- & 0 & -- \\
\hline & & ASTM D154 & 1970 & - & $O(T M)$ & -- \\
\hline & & ASTM D333 & 1968 & -- & $O(T M)$ & -- \\
\hline & & ISO R1516 & 1970 & -- & $\mathrm{S}$ & -- \\
\hline & & ISO RI517 & 1970 & -- & $O(T M)$ & -- \\
\hline & & ISO RI518 & 1970 & -- & $O(T M)$ & -- \\
\hline & & ISO RI519 & 1970 & -- & $O(T M)$ & - \\
\hline & & ISO RI520 & 1970 & -- & $O(T M)$ & - \\
\hline & & ISO RI521 & 1971 & -- & $O(T M)$ & -- \\
\hline & & ISO RI523 & 1971 & -- & $S(T M)$ & -- \\
\hline & & ISO RI524 & 1971 & -- & 0 & - \\
\hline & & ASTM D2832 & 1969 & -- & 0 & -- \\
\hline & & ASTM D2206 & 1968 & -- & $O(T M)$ & -- \\
\hline & & ASTM D714 & 1970 & -- & 0 & -- \\
\hline & & ASTM D2366 & 1968 & -- & $P(T M)$ & -- \\
\hline & & ASTM D1361 & 1970 & -- & $S(T M)$ & -- \\
\hline & & ASTM D2369 & 1968 & -- & $S(T M)$ & -- \\
\hline & & ASTM D2486 & $1969 \mathrm{~T}$ & -- & $P(T M)$ & - \\
\hline & & ISO 2409 & 1972 & -- & $O(T M)$ & -- \\
\hline & & ISO 2431 & 1972 & -- & $P$ & -- \\
\hline 0908 & Thinners, paint and varnish & ASTM B2349 & 1968 & -- & 0 & -- \\
\hline 0909 & Adhesives and adhesive products & & & & & \\
\hline & including glues & ASTM D1779 & 1970 & -- & 0 & - \\
\hline & & PI $3 p 54$ & $\begin{array}{l}\text { Proposed } \\
1954\end{array}$ & -- & $\begin{array}{c}0 \\
\text { (test proc.) }\end{array}$ & -- \\
\hline & & ASTM D2851 & 1970 & -- & 0 & -- \\
\hline & & ASTM D2559 & 1970 & -- & 0 & - \\
\hline & & ASTM C557 & 1967 & -- & 0 & -- \\
\hline & & ASTM D816 & 1970 & -- & $O(T M)$ & -- \\
\hline & & ASTM D897 & 1968 & -- & $O(T M)$ & -- \\
\hline & & ASTM D1151 & 1969 & - & $P(T M)$ & -- \\
\hline & & ASTM D950 & 1970 & -- & $O(T M)$ & -- \\
\hline & & ASTM D2095 & 1969 & -- & $O(T M)$ & -- \\
\hline & & ASTM D1084 & 1970 & -- & $O(T M)$ & - \\
\hline & & ASTM D1916 & 1969 & $\begin{array}{l}\text { ANSI Z199.1 } \\
\quad(1970)\end{array}$ & $O(T M)$ & -- \\
\hline & & AVATI $* 15$ & 1968 & -- & 0 & -- \\
\hline & & AVATI *3 & 1951 & -- & 0 & - \\
\hline & & AVATI $* 4$ & 1961 & -- & 0 & -- \\
\hline
\end{tabular}




\begin{tabular}{|c|c|c|c|c|c|c|}
\hline $\begin{array}{l}\text { Product } \\
\text { Number }\end{array}$ & $\begin{array}{l}\text { Product } \\
\text { Description }\end{array}$ & $\begin{array}{c}\text { Responsible Organi- } \\
\text { zation and Standard } \\
\text { Designation }\end{array}$ & $\begin{array}{l}\text { Latest } \\
\text { Issue or } \\
\text { Status of } \\
\text { Standard }\end{array}$ & $\begin{array}{l}\text { Standard } \\
\text { Accepted by }\end{array}$ & $\begin{array}{l}\text { Type of } \\
\text { Standard }\end{array}$ & $\begin{array}{l}\text { Certifi- } \\
\text { cation } \\
\text { Program } \\
\end{array}$ \\
\hline \multirow[t]{3}{*}{ P0910 } & \multirow[t]{3}{*}{ Gasoline } & ASTM D439 & 1970 & $\begin{array}{l}\text { ANSI } \mathrm{Zl1} .285 \\
(1971)\end{array}$ & $P$ & -- \\
\hline & & ASTM D526 & 1970 & $\begin{array}{l}\text { ANSI Z11.48 } \\
\quad(1971)\end{array}$ & $O(T M)$ & -- \\
\hline & & ASTM D2533 & 1972 & $\begin{array}{l}\text { ANSI Z11.221 } \\
\quad(1968)\end{array}$ & $O(T M)$ & -- \\
\hline 0911 & Kerosene & ASTM D187 & 1968 & $\begin{array}{l}\text { ANSI } \mathrm{Z11.17} \\
(1971)\end{array}$ & $P(T M)$ & -- \\
\hline \multirow[t]{15}{*}{0912} & \multirow[t]{15}{*}{$\begin{array}{l}\text { Antifreeze, windshield wiper } \\
\text { fluid }\end{array}$} & ASTM D1880 & 1970 & -- & 0 & -- \\
\hline & & ASTM DII20 & 1965 & CSMA & $O(\mathrm{TM})$ & - \\
\hline & & ASTM D1882 & 1966 & CSMA & $O(T M)$ & -- \\
\hline & & ASTM DII77 & 1965 & CSMA & $O(T M)$ & -- \\
\hline & & ASTM DII22 & 1968 & CSMA & $O(T M)$ & -- \\
\hline & & ASTM DIII9 & 1965 & CSMA & $O(T M)$ & -- \\
\hline & & ASTM D1287 & 1965 & CSMA & $O(T M)$ & -- \\
\hline & & ASTM DI121 & 1967 & CSMA & $O(T M)$ & -- \\
\hline & & ASTM D1123 & 1968 & CSMA & $O(T M)$ & -- \\
\hline & & ASTM D1384 & 1970 & CSMA & $P(T M)$ & -- \\
\hline & & ASTM D1881 & 1967 & CSMA & $\mathrm{P}(\mathrm{TM})$ & -- \\
\hline & & ASTM D2570 & 1970 & CSMA & $P(T M)$ & -- \\
\hline & & ASTM D2758 & $1968 \mathrm{~T}$ & CSMA & $\mathrm{P}(\mathrm{TM})$ & -- \\
\hline & & ASTM D2847 & 1969 & CSMA & $P(T M)$ & -- \\
\hline & & ASTM D1124 & 1968 & CSMA & $\mathrm{P}(\mathrm{TM})$ & -- \\
\hline \multirow[t]{11}{*}{0913} & \multirow[t]{11}{*}{$\begin{array}{l}\text { Lubricants, machine oils, } \\
\text { engine oils }\end{array}$} & ASTM D1367 & 1968 & $\begin{array}{l}\text { ANSI Z11.138 } \\
(1971)\end{array}$ & $O(T M)$ & -- \\
\hline & & ASTM D217 & 1968 & $\begin{array}{l}\text { ANSI Z11.3 } \\
(1969)\end{array}$ & $O(T M)$ & -- \\
\hline & & ASTM D2265 & 1967 & $\begin{array}{l}\text { ANSI Z11.207 } \\
(1968)\end{array}$ & $O(T M)$ & -- \\
\hline & & ASTM D2625 & 1969 & $\begin{array}{l}\text { ANSI } \mathrm{Z11} .282 \\
(1970)\end{array}$ & $O(T M)$ & -- \\
\hline & & $\begin{array}{l}\text { ASTM D2595 } \\
\text { ASTM D2509 }\end{array}$ & $\begin{array}{l}1970 \\
1968\end{array}$ & $\begin{array}{l}\text { ANSI } \overline{\mathrm{Zl}} .235 \\
\quad(1969)\end{array}$ & $\begin{array}{l}O(T M) \\
O(T M)\end{array}$ & $\begin{array}{l}-- \\
--\end{array}$ \\
\hline & & ASTM D2782 & 1971 & -- & 0 & -- \\
\hline & & ASTM D2783 & 1971 & $=-$ & 0 & -- \\
\hline & & ASTM D128 & 1968 & $\begin{array}{l}\text { ANSI Z11.16 } \\
(1971)\end{array}$ & 0 & -- \\
\hline & & ASTM D2266 & 1967 & $\begin{array}{l}\text { ANSI Z11.208 } \\
(1968)\end{array}$ & $O(T M)$ & -- \\
\hline & & SAE J183 & 1971 & -- & PO & -- \\
\hline & & NLGI & 1970 & $\begin{array}{l}\text { ANSI Z11.130 } \\
\quad(1971)\end{array}$ & 0 & -- \\
\hline 0915 & Methyl alcohol, solvent & ASTM D770 & 1970 & - & 0 & -- \\
\hline \multirow[t]{4}{*}{0916} & \multirow[t]{4}{*}{ Caustics, lye } & ASTM D456 & 1970 & $\begin{array}{l}\text { ANST K60.10 } \\
(1969)\end{array}$ & 0 & -- \\
\hline & & AWWA B501 & 1964 & -- & 0 & -- \\
\hline & & ASTM E291 & 1971 & -- & 0 & -- \\
\hline & & TAPPI T613 & 1970 & -- & 0 & -- \\
\hline 0917 & Charcoal & $\begin{array}{l}\text { ASTM D } 2677 \\
\text { ASTM D1762 }\end{array}$ & $\begin{array}{l}1971 \\
1968\end{array}$ & -- & $O(T M)$ & -- \\
\hline 0918 & Pins and needles & CSWA $\mathrm{V} .1$ & 1970 & - & $\mathrm{P}$ & -- \\
\hline \multirow[t]{3}{*}{0919} & \multirow[t]{3}{*}{ Rope and string } & CI *I & 1971 & -- & 0 & -- \\
\hline & & $C I * 2$ & 1972 & -- & 0 & -- \\
\hline & & ASTM D738 & 1958 & $\begin{array}{l}\text { ANSI } 114.45 \\
(1960)\end{array}$ & $O(T M)$ & -- \\
\hline
\end{tabular}




\section{P0920 Caulking/spackling compounds}

0921 other chemicals

\section{Wellpaper cleaners and removers, including steamers \\ 0923 Cleaning equipment, including brooms, mops, brushes, cleaning pads, buckets, wash tubs, etc.}

0924 Paint and varnish removers

0925 Paint brushes and rollers

0926 Photographic chemicals
ASTM D739

ASTM D1233

ASTM D2450

ASTM D2452

ASTM D2453

ASTM D2202

ASTM D2203

ASTM D2377

ASTM $\mathrm{C} 61$

ASTM $\mathrm{Cl}_{4} 04$

ASTM $\mathrm{C} 476$

ASTM C6

ASTM C206

ASTM C207

USC CS69

CAOAC (chapter 5)

UL 41

ASTM C91

ASTM C94

ASTM C494

1958

1958

1969

1969

1969

1966

1966

1967

1964

1970

1971

1968

1968

1968

1938

1965

1971

1971

1971

1971

ANSI PH4 .100 thru

$$
\mathrm{PH} 4.304
$$

ISO $\mathrm{R} 420$

ISO R423

ISO $\mathrm{R} 424$

ISO R418

ISO $R 419$

ANSI PH4.125

ASTM D335
ASTM D773
ASTM D119
CPSTC 1 thru 58
ASTM D1000
ASTM D2860
ASTM D2148
ASTM D69
ASTM D2754

1966-72

1965

1965

1965

1965

1965

1964

1951

1970

1970

$1970(?)$

1972

1970

1968

1967

1970 $\begin{array}{ll}-- & O(T M) \\ - & O(T M)\end{array}$

-- $\quad O(T M)$

$\therefore \quad O(T M)$

-- $\quad 0(\mathrm{TM})$

-- O(TM)

-- $\quad O(T M)$

$--$

ANSI A66.I

(1964)

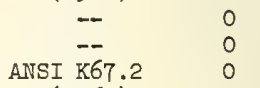

(1969)

ANSI K67.12 0

(1960)

ANSI K67.13 0

(1969)

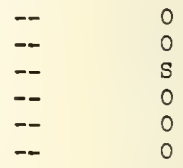

$--$

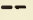

ANSI $\mathrm{PH}_{4} .126$

(1972)

ANSI $\overline{\mathrm{PH}} 4.275$

(1972)

ISO Rì22

(1965)

ANSI
(1969) 59.39

TAPPI T463m

(1952)

ANSI C59.6

(1968)

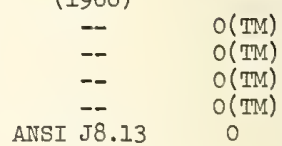

(1971)

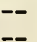

$--$ 
FARM SUPPLIES AND EQUIPMENT

P1001. Electric fences
1002 Home pasteurizers
1003 Cream separators

\section{PACKAGING AND CONTAINERS FOR HOUSEHOLD PRODUCTS}

1101 Pressurized containers, aerosol cans
UL 69

CSMA AG

ASTM D3088

ASTM D3089

ASTM D3092

ASTM D3093

ASTM D3094

ASTM D3095

ASTM D3096

ASTM D3061

ASTM D3068

ASTM D3069

ASTM D3071

ASTM D3072

ASTM D3074

ASTM D3075
1971

1972

1972

1972

1972

1972

1972

1972

1972

1972

1972

1972

1972

1972

1972

1102 Vacuum containers, "thermos containers"

1103 Self-contained openers, "pop-top" cans, zip-open cans, etc.

1104 Resealable closures

1105

Child-resistant closures

1106

Glass bottles and jars, except soft drink bottles

\section{USC R10 \\ ASTM Cl 47 \\ ASTM C148 \\ ASTM C225 \\ ASTM Cl 49 \\ CCSMA AG}

TAPPI T809

TAPPI T810

USC CS192

ASTM D2141

ASTM D1709

ASTM D2103

ASTM D2578

NSF 31
1947

1969

1971

1968

1971

1971

1971

1966
1107 Other containers, except vacuum

1108 Plastic wrapping products, including trash, clothes and garden bags
1953

1968

1972

1972

1967

1970
1969

1961

1972

972

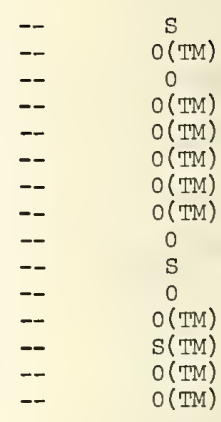

--
--
--
--
--
--
--
--
--
--
--
--
--
--
--

Standard

Type of

Certifi-

cation

Program
S

UL

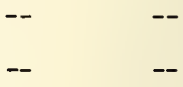

$-$
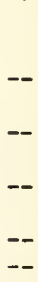

\section{S}

S

$\begin{array}{ccc}-- & 0 & -- \\ -- & 0(\mathrm{TM}) & -- \\ -- & 0 & -- \\ -- & 0(\mathrm{TM}) & -- \\ -- & 0(\mathrm{TM}) & -- \\ -- & \mathrm{S} & --\end{array}$

$-$

0

0

$-$

$\begin{array}{ccc}-- & 0 & - \\ \text { ANSI K65.132 } & 0(\text { TM }) & - \\ (1971) & & \\ -- & 0(T M) & - \\ -- & 0 & - \\ \text { ANSI K65.129 } & 0(\text { TM) } & - \\ (1971) & & - \\ -- & 0 & -\end{array}$


Paper wrapping products, paper objects including paper towels, tissues, newspapers, stationery, etc.

1110 Glass soft drink bottles

NSDA * 1

USC

1111 Containers, plastic

USC TS125c ASTM D2659

ASTM D2463

ASTM D2561

ASTM D2684

SPI PBDI

SPI PBD2

SPI PBD3

SPI PBD4

SPI PBD5

SPI PBD6

SPI PBD7

SPI PBD8

1112 Containers, metal (cans)
ANSI MH2
UL 30

ISO R9O

CMI *1-11

FMS 6051 and 6052

CCTI B-2ll.2

CCTI B-2l1.3

CCTI B-2l1.4

CCTI B-401.3

1967
1955
1963
1964
1964
1942
1940
1971
1970
1964
19661
1969
1947
1969
1964
1962
1962

1970

IP 1972

IP 1970

1967

1970

1970

$1968 \mathrm{~T}$

1966

1968

1968

1968

1968

1968

1969

1969

1968

1971

1959

1967

1966

1972

1972

1972

1972

1113 Bags, oven cooking/roasting

1114 Cardboara boxes, cartons and other cardboard products

$$
\begin{aligned}
& \text { TAPPI T808 } \\
& \text { TAPPI T807 } \\
& \text { TAPPI } \mathrm{T} 452 \\
& \text { TAPPI T457 } \\
& \text { TAPPI T461 } \\
& \text { TAPPI T489 } \\
& \text { TAPPI T492 } \\
& \text { TAPPI T494 } \\
& \text { TAPPI T495 } \\
& \text { TAPPI T506 } \\
& \text { TAPPI T510 } \\
& \text { TAPPI T401 }
\end{aligned}
$$


TAPPI T404

TAPPI T411

TAPPI T410

TAPPI T433

PPC *1

PI 110.2

PI 110.6

PI 110.8

PI 110.11

PI 110.12

PI 110.13

PI 110.7

PI T3608p

PI T3609p

PI T3610p

PI T3612p

PI T3614p

PI T3615p

PI T3618p

PI T3619p

PI T3620p

PI T3635p

P1115 Aluminum foil wrapping products

CAA *28 (foil)

ASTM E345
$1966 \mathrm{~T}$

1968

1968

1944

1964

1962

1962

1963

1963

1963

1963

1963

1962

1962

1962

1962

1962

1962

1962

1962

1962

1962

1972-73

1969

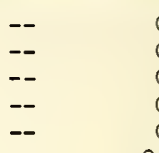

$-$

$-$

$-$

--

$--$

$-$

$--$

--

$-$

$--$

$-$

$-$

$--$

-

$-$

$--$

$--$

o(TM)

0
0
0
0
0
$0(T M)$

$O$ (TM)

$O$ (TM)

$O$ (TM)

$O$ (TM)

$O(T M)$

$O$ (TM)

O (TM)

$O(T M)$

$O$ (TM)

O (TM)

$O(T M)$

$O$ (TM)

$O$ (TM)

$O(T M)$

$O(T M)$
$O(T M)$

SPORTS AND RECREATIONAL EQUIPMENT

1201 Playground/yard equipment not elsewhere classified, including sandboxes, simulated forts, towns, spaceports, ete.

CUSC

IP 1972

1972

1972

$-$

S

TM

BMA BMA $6 T$

MPPA 302

CABYC

CBIA EM

CBIA HEM

UL 1149

UL 1135

UL 1112

UL 1133

UL 1123

UL 1102

UL 1121
1968

1968
$1972-73$
1971
1971
IP 1971
IP 1971
IP 1971
IP 1971
1972
1972
1972

ANSI $\mathrm{z} 120.1$ (1968)

$-$

-- SPO

-- SPO

--

$-$

$--$

$-$

$-$

$--$

$-$

1204 Baseball equipment, apparel, basebells, bats, "bases," masks and other protective gear, etc.

1205 BasketbalI equipsent

1206 Bowling equipment

1207 Boxing equipment

1208 Croquet equipment 


\section{P1209 Exercise equipment, including} exercise bikes, weights, boards, rollers, etc.

1210 Fishing equipment, including poles, lines, lures, hooks, fishing knives, scalers, nets, tackle box, ete.

1211 Football equipment, including apparel and footware, protective gear, football "kicking tee," blocking sleds, tackling sleds, tackling and blocking dumies, goalposts

1212 Golf equipment except golf carts, including golf balls, clubs, bags, shoes

1213 Golf carts

1214 Hockey equipment, includes apparel and protective gear, and all equipment

1215 LaCrosse equipment

1216 Snow skiiing equipment, including skis, poles, boots, and binding ASTM Committee F8

$1217 \quad$ Sleds

1218 Snowmobiles

1219 Tennis and badminton equipment, including nets and standards, racquets, tennis balls, "shuttle cocks", etc.

1220

Swimming and sports equipment, including life preservers, snorkling equipment

1221 Headgear for cycling - helmets

ANSI Z90.1

SNG *I

MIC *I
1971

1970

1967

Beach equipment, including unbrellas, mats, etc.

Stationary outdoor grills kerosene

Portable gasoline cooking stoves/grills

Portable gasoline heating equipment

Gasoline, kerosene and propane lanterns and lamps 
Product. Product

Number

Description

nd Standard Designation

P1228 Picnic equipment, including picnic baskets, coolers, etc. USC PS49

1229 Camping equipment, including

tents, tent stakes, cots,

camp mattresses, adult sleeping

bags, hammocks, mosquito

netting, portable sanitary

facilities

NFPA 224

1969

1230 Camping trailers, and related equipment, not mobile homes
IAPMO TSC9

IAPMO TSC1

IAPMO TSC2

IAPMO TSC4

IAPMO TSC8

IAPMO TSC11

IAPMO TSCI2

IAPMO TSC13

IAPMO TSCI 4

IAPMO TSC15

IAPMO TSC18

IAPMO TSC19

UL 307b

UL 311

NFPA 501 c

ANSI Z21.57
1971

\section{2}

1967

1971

1971

1970

1972

1965

1966

1972

1966

1968

1971

1970

1971

1970

1972

1972

1972

1969

1970

1969

1971

1953

1966

1966

1972

ASTM Committee F8

IP 1972

AMO *1

1972

1235 Archery equipment

1236

Unlicensed motor scooters and go-carts

1237 Gas, air and spring operated guns

1238 Specialized clothing for camping or sports, not elsewhere classified

1231 Swimming pools and associated equipment, excluding above

(ats

UL 676
NSF 27
NSF 38
NSF 28
NSF 39
NCMA *11
NSF 17
NSF 19

$-$

(n)

$-$
Standard

Type of

Certifi-
Horseback riding equipment and accessories, including saddles, pads, bridies, apparel, etc. 
P1240 Aquariums and accessories, pumps,
heaters, lights, filters, etc.

P1240 Aquariums and accessories, pumps,
heaters, lights, filters, etc. CGTA

1241 Swings/swing sets CUSC

1242 Slides CUSC

1243 See saws

CUSC

1244 Playground climbing apperatus, "monkey bars," "gyms"

CUSC

1245 Ice skates

1246 Wading pools

1247 Charcoal igniter fuel

1248 Darts and dart games, lawn darts, targets, etc.

1249 Stationary "built-in" grills, charcoal

1250 Stationary "built-in" grills, gas

1251 Portable grills, kerosene

1252 Portable grills, charcoal "Hibachi"

1253 Portable grills, gas (LP)

1254 Portable stoves, kerosene

1255 Portable alcohol heating equipment

1256 Portable gas (LP) heating equipment

1257 Horseshoes

1258 Mountain climbing equipment, including picks, spikes, apparel, etc.

1259 Outdoor grills, not otherwise specified

1260 Billiards, tables, balls, cues, chalk, "pool table" BCA *1

$\begin{array}{llll}1969 & -- & \text { SP } & \text { GTA } \\ \text { IP } 1972 & -- & \text { S } & -- \\ \text { IP } 1972 & -- & \text { S } & -- \\ \text { IP } 1971 & -- & \text { S } & --\end{array}$

IP $1972 \quad--\quad$ S
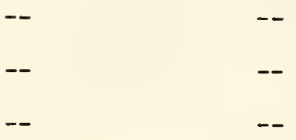

$-$

$--$

$--$

$--$

$-$

$-$

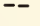

$-$

$--$

1261 Surfboards

1262 Swimming pools, above ground, including all associated equipment - frame, filter, pump, etc.

1263 Minibikes

1264 Water skiing equipment

1265 Sports balls, not otherwise specified 
Product Product Number Description

P1266 Volleyballs

1267 Soccer equipment and apparel

1269 Ping pong - tables, nets, paddles, balls

1271 Batons

1272 Gymnastics equipment, including mats, rings, parallel bars, uneven bars, balance beams
Responsible Organi- Issue or zation and Standard Status of Designation

\section{Standard}

1964 1964 1964 1964 1964

ISO R381

ISO R38?

\section{Toboggans}

1274 Snow "disks" and similar items

1275 Scuba diving equipment - masks, air tanks, apparel and other gear

TOYS

1301 Tricycles

CUSC

1302 Unpowered dolls and toy animals

1303 Mechanical dolls and toy animals including keywind and battery operated

CUSC

1304 Windup and battery operated toys not otherwise specified

$\subset \mathrm{USC}$

cUsC

flying airplanes, boats, not including models

1306 Gasoline powered toys, including model airplanes and cars

1307 Electric games

CUL 696

1308 Roller skates

CUSC

1309 Kites and kite string

CUSC

1310 Pogo sticks

CUSC

1311 Toy guns and other toy weapons without projectiles

cUsC

1313 Fireworks

1314 Rocketry sets
CUSC

Toy guns and other toy weapons with projectiles

CUse

NFPA 4IL NAR *1

$\subset \mathrm{USC}$
IP 1972

IP 1972

IP 1972

IP 1972

IP 1972

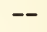

$\mathrm{S}$

$\mathrm{S}$

1966

ANSI C33.61

(IP 1972)

IP 1972

IP 1972

IP 1972

$\mathrm{S}$

IP 1972

S

IP 1972

1968

1970

IP 1972
Certifi- 
P1315 Chemistry sets

1316 Science kits and toys, not otherwise specified

1317 Flying devices not gasoline or rocket powered cUSC

IP 1972

1318 Models and their construction materials, not otherwise specified

cusc

IP 1972

Accepted by

1319 Metal and plastic molding sets CUSC

IP 1972

1320 Games, non-electric

1321 Toy home equipment, including stoves, sinks, irons, sewing machines, washing machines, non-heat ovens, etc.

cusc

IP 1972

CUSC

IP 1972 other play enclosures

1323 Puzzles

1324 Toy balls

CusC

IP 1972

1325 Inflated toys other than balloons CUSC

IP 1972

1326 Blocks, pull-toys and similar items

CUSC

IP 1972

1327 Rocking, hobby horses, and similar child-carrying toys, not wheeled

CUSC

IP 1972

1328 Wagons, toy

cusc

IP 1972

1329 Scooters

cusc

IP 1972

1330 Powered riding toys, not including bicycles

1331 Other riding toys, not powered, not including bicycles

$\subset \mathrm{USC}$

IP 1972

NMRA ( 10 standards and 13 recommended practices) cUL 696

cusc

1971

1971

IP 1972

ANSI C33.61

(IP 1972)

IP 1972

cusc

CUL 696

CUSC

1971

IP 1972

CUL 696

cusc

1971

IP 1972

IP 1972

cusc

$\begin{array}{lll}-- & \text { S } & -- \\ -- & \text { S } & -- \\ -- & \text { S } & \text { UL } \\ -- & \text { S } & -- \\ -- & \text { S } & \text { UL } \\ -- & \text { S } & -- \\ -- & \text { S } & --\end{array}$




\begin{tabular}{|c|c|c|c|c|c|c|}
\hline P1337 & Toy medical equipment & CUSC & IP 1972 & -- & S & - \\
\hline 1338 & Toy bows and arrows & $\subset \mathrm{USC}$ & IP 1972 & -- & S & - \\
\hline 1339 & Doll houses and doll furniture & CUSC & IP 1972 & -- & S & - \\
\hline 1340 & $\begin{array}{l}\text { Doll carriages, strollers, } \\
\text { cribs, etc. }\end{array}$ & CUSC & IP 1972 & -- & S & - \\
\hline 1341 & Juvenile sports equipment & -- & -- & -- & -- & - \\
\hline 1342 & $\begin{array}{l}\text { Costumes (Halloween, etc.) } \\
\text { including masks, wigs }\end{array}$ & CUSC & IP 1972 & -- & $S$ & - \\
\hline 1343 & Toy make-up kits & $\subset \mathrm{USC}$ & IP 1972 & -- & S & - \\
\hline 1344 & Toy musical instruments & 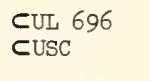 & $\begin{array}{l}1971 \\
\text { IP } 1972\end{array}$ & -- & $\begin{array}{l}S \\
S\end{array}$ & UL \\
\hline 1345 & $\begin{array}{l}\text { Building sets ( Lincoln log, } \\
\text { tinker toys, erector sets, etc.) }\end{array}$ & ¿USC & IP 1972 & -- & $\mathrm{S}$ & - \\
\hline 1346 & Clacker balls & $\subset \mathrm{USC}$ & IP 1972 & -- & S & -- \\
\hline 1347 & Balloons & CUSC & IP 1972 & -- & S & - \\
\hline 1348 & $\begin{array}{l}\text { Caps and cap toys (guns, rifles, } \\
\text { rockets, cannons) }\end{array}$ & $\subset \mathrm{USC}$ & IP 1972 & -- & $\mathrm{S}$ & - \\
\hline 1349 & Stilts & $\subset \mathrm{CUSC}$ & IP 1972 & -- & S & -- \\
\hline 1350 & Squeeze/squeaker toys & $\subset \mathrm{USC}$ & IP 1972 & -- & S & -- \\
\hline 1351 & Crayon and children's paint sets & $\begin{array}{l}\text { USC R192 } \\
\text { CUSC }\end{array}$ & $\begin{array}{l}1963 \\
\text { IP } 1972\end{array}$ & -- & $\begin{array}{l}0 \\
S\end{array}$ & -- \\
\hline 1352 & $\begin{array}{l}\text { Slingshots, "sling propelled" } \\
\text { toys }\end{array}$ & CUSC & IP 1972 & -- & $\mathrm{S}$ & -- \\
\hline 1353 & Toy boxes or chests & cUsC & IP 1972 & -- & $\mathrm{S}$ & - \\
\hline 1354 & Marbles & cusc & IP 1972 & -- & $\mathrm{S}$ & -- \\
\hline 1355 & Toy dishes, tea sets & CUSC & IP 1972 & -- & $\mathrm{S}$ & -- \\
\hline 1356 & $\begin{array}{l}\text { Fuel for model cars, airplanes, } \\
\text { etc. }\end{array}$ & CUSC & IP 1972 & -- & $\mathrm{S}$ & -- \\
\hline 1357 & Mechanical drawing toys & cusc & IP 1972 & -- & $S$ & -- \\
\hline 1358 & $\begin{array}{l}\text { Children's blackboards and } \\
\text { easels }\end{array}$ & $\begin{array}{l}\text { ASTM C543 } \\
\text { USC PS2 } \\
\text { CUSC }\end{array}$ & $\begin{array}{l}1967 \\
1966 \\
\text { IP } 1972\end{array}$ & $\begin{array}{l}-- \\
--\end{array}$ & $\begin{array}{l}0 \\
0 \\
S\end{array}$ & $\begin{array}{l}-- \\
-- \\
--\end{array}$ \\
\hline 1359 & Toy jewelry & CUSC & IP 1972 & -- & $\mathrm{S}$ & - \\
\hline 1360 & Children's sleeping bags & -- & -- & -- & -- & -- \\
\hline 1361 & $\begin{array}{l}\text { Toy parking garages, service } \\
\text { stations, spaceports, farm } \\
\text { yards and similar toys }\end{array}$ & cusc & IP 1972 & -- & $S$ & -- \\
\hline 1362 & Woodburning kits & CUL 696 & 1971 & -- & $\mathrm{S}$ & UL \\
\hline
\end{tabular}


P1363 Whistles

$\subset \mathrm{USC}$

1364 Doll clothes and accessories

cusc

1365 Water/swimming toys

$\subset \mathrm{USC}$

1366 Children's books

1367 Toy banks

CUSC

IP 1972

1368 Tracks for toy cars, not electric

СUsC

YARD AND GARDEN EQUIPMENT

1401 Power mowers, not specified

ANSI B71.I

CUL 82

CUL 73

1402 Hand mowers

1403 Hand garden tools

1404 Power lawn trimmers and edgers

CUL 73

$\subset \mathrm{UL} 82$

1405 Garden tractors

1406 Snow throwers and snow plows

СUL 73

CUL 82

1407 Garden sprayers

1408 Power tillers and cultivators

1409 Other power garden tools

1410 Outdoor lighting equipment, electric

\section{Chain saws}

1412 Pumps, including electric submersible fountain pumps
СWSC

AWWA EI01
CUL 82

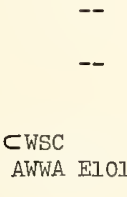

1972
IP 1972
1972

1972

IP 1972

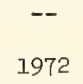

IP 1972

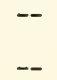

IP 1972

1971

1971

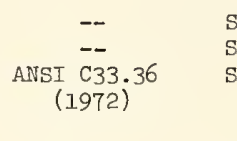

S S

UL

UL

(1972)
C33.

$\begin{array}{lll}-- & S & - \\ -- & S & - \\ -- & S & - \\ -- & -- \\ -- & \text { S } & --\end{array}$

S

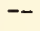



Designation

Standard

\section{P1417 Yard decorative equipment}

1418 Power mowers, rotary, gasoline

1419 Power mowers, rotary, electric

1420 Power mowers, reel, gasoline

1421 Power mowers, reel, electric

1422 Power mowers, riding

1423 Manual edgers

1424 Manual pruning and trimming equipment

1425 Wheelbarrows/lawn carts

1426 Hatchets, axes

1427 Powered hedge trimmers

1428 Patio lights/torches, fuel burning

1429 Plant food, fertilizer

1430 Seed and fertilizer spreaders

1431 Grass/leaf catcher attachments for mowers

1432 Animal traps

1433 Powered leaf mulchers/grinders, "shreader-baggers"

\section{CHILD NURSERY EQUIPMENT AND SUPPLIES}

1501 Highchairs

1502 Changing tables

1503 Infant seats

1504 Cribs, including springs and mattresses

1505 Carriages

1506 Gates

1507 Beby blankets, sheets, pads, pillows, and other baby bedding equipment

1508 Baby walkers

1509 Bottles, nipples

1510 Bottle warmers

CBHMA 1201

ANSI B71.1

ANSI B71.1

CUL 82

ANSI B71.1

ANSI B71.1

ANSI B71.1

1970

1972

1972

IP 1972

1972

1972

1972

CAOAC (chapter 2)

1965
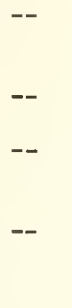
CCEE 11, pt.1 and pt. 2 , sec. $B$ CUL 499

Diapers, including disposable

\section{diapers \\ 1512}

Playpens

1513

Pery baths

1514 Baby baths

1515 Baby scales

1516 other nursery furniture and equipment

1517 Baby rattles

1518 Youth chairs

1519 Car seats

1520 Baby exercisers

1521 Baby swings

1522 Strollers

1523 Diaper pins

1524 Baby harnesses

1525 Pacifiers, teething rings

1526 Crib mobiles, crib gyms

1527 Infant "carriers," personal use (back packs, slings, etc.)

1528 Diaper pails

1529 Portable cribs

1530 Bathinettes

1531 Baby carriers - bicycle accessory

\section{PERSONAL USE ITEMS}

1601

\section{Razors and shavers, razor} blades

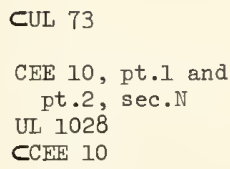

1602 Hair aryers

UL 859

AHAM

CCEE 10

1603 Hair curlers, pins, clips, etc. UL 859

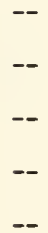

\section{6} 1972

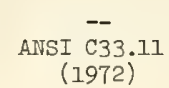

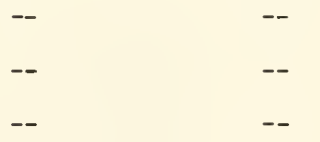
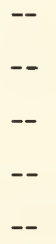

\section{2}

ANSI 033.36

UL

$$
\text { (1972) }
$$

$1964 \& 66$ IP 1971 1960

1966
IP 1972
1960

1966

1970

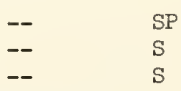

CEE

UL

CEE

ANSI C33.33 (1966)

--

ANSI C33.33

(1971)
UI

$--$ UL 
SAE J563a

1605 Wigs, falls and other hair pieces

1606 Eyeglasses

ANSI Z80.1

1607 Eye protection devices, including sunglasses and light shields

ANSI 287.1

CUL 73 "piks"

CUL 73

CEE 10, pt.I and pt. 2 , sec.0

CCEE 10

ANSI Z21.55

CUI 499

CUL 73

USC $\operatorname{cs} 234$

USC CSI87

USC CS191.

usc CS166

USC CS137

USC CS46

USC CS15

ASTM D1230

USC PS45

USC PS36

1615 Footwear including shoes, boots, slippers, sandals, sneakers

ANSI 241.1

ASTM D2322

ASTM D1630

1616 Jewelry

1617 Ear protection devices, including noise plugs

ANSI 224.22

ANSI 288.2

1618 Respiratory protection devices, gas mask, aspirator, etc.
1969

1972

1968

1972
1972

1964866

1960

1971

1972

1972

1961

1952

1953

1950

1951

1965

1946

1961

1971

1970

1967

1969

1968

ALCA É $\overline{61}$

(1969)

ANSI J2.26

(1971)

(1972)

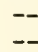

(1972)

(1963) and

AATCC 33

(1962)

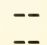

0

ANSI C 33.36

S

(1972)

ANSI C33.36 S

SP

$S$

CEE

CEE

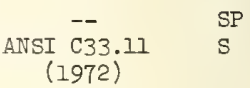

ANSI C33.36

ANSI II4.69

1971

S

1969
$\mathrm{S}$

0

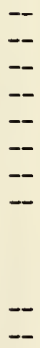

AGA

UL

UL

$\mathrm{S}$ o(TM) $O(T M)$

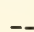

1619 Personal protection devices, including tear gas guns, pens, etc. 
P1620 Hearing aids

1621 Umbrellas, personal use

1622 Wrist watches, pendant watches, pocket watches

ANSI 53.8

ANSI 53.3

HAIC *1

ISO R2281

1972

1623 Luggage

1624 Contact lenses

1625 Lighted make-up mirrors

1626 Pens, pencils and other desk supplies, including paper clips, rulers, yardsticks, erasers, rubber bands

CUT

CSWA v.1 (paper clips) 1970

1627 Hair curlers, electric with steam

1628 Hair curlers, electric without steam

1629 Non-powered tooth brushes

1631 Grocery carts and luggage carriers, personal use

1632 Sewing basket articles

1633 Personal use hygiene items

1634 Heat lamps

\section{CUL 73 \\ UL 482 \\ CUL 499}

ANSI 280.2

1972

1970

ANSI $\mathrm{X} 4.12$
$(1970)$

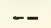

$\begin{array}{ll}-- & P \\ -- & 0 \\ -- & 0\end{array}$

Interim

1965
-

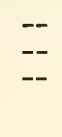


P1645 Day wear (underwear, skirts, slacks, blouses, shirts, dresses, socks/hose, suits, sweaters, vests)

\section{CANSI L22}

USC CS46

USC CS234

CANSI L22

1647 Clothing accessories (gloves/ mittens, scarves, hats/caps, handbags, belts, wallets/ billfolds, etc.)

\section{MISCELIANEOUS PRODUCTS}

1701 Christmas trees, artificial

1702 Switchblade, gravity and pocket knives

1703 Mobile homes and related equipment

\section{CAISI 221.11 .1 \\ CAHSI Z21.42 \\ CANSI 221.44 \\ CANSI Z21.47 \\ CANSI Z21.48 \\ CAINSI $\mathrm{Z} 21.49$ \\ UL 311 \\ IAPMO TSC9
NFPA 501B \\ NPA 1 \\ NFPA $501 \mathrm{C}$ \\ IAPMO TSCI \\ ANSI Z21.1.I \\ ANSI Z21.1.2 \\ ANSI 221.10 .1 \\ ANSI $221 \cdot 10.3$ \\ UL 307 (a) \\ Ut $307(\mathrm{~b})$ \\ IAPMO TSCB \\ IAPMO PSE \\ IAPMO PST \\ IAPMO PSI 4 \\ IAPMO PS23 \\ ASTM DI 862}

1968

1968

1965

1961

1971

1971

1971

1971

1967

1967

1971

1972

1971

1971

1970

1967

1967

1967

1971

1971

1969

1965

1970

1966

1966

1971

1968

1971

-




\section{Product Product \\ Number Description \\ Pl710 Home first-aid and health equipment}

1711 Christmas tree lights

UL 588

1712 Christmas tree stends and supports

1713 Christmas tree ormaments and other decorations

1714 Other seasonal decorations, Easter baskets, Halloween decorations, etc.

1715 Pet supplies, pet cages, pet carriers, leashes, collars, etc.

1716 Hypodermic needles and syringes

ISO R595

ISO R596

1717 Breces, orthopedic

1718 Fmergency flares, signal flares SAE J597

1719 Lunch boxes/pails

1720 Party favors

1721 Adult games and novelty items

HOME STRUCTURES, CONSTEUCTION MATERIALS

1801 Stairs, ramps, and lendings, indoors and outdoors

1802 Fireplaces, individuolly built

1803 Insulation materials

1804

Siding materials, including aluminum siding

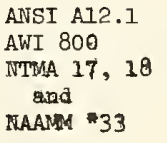

NFPA 211

SIGMA 65-7-2

USC R179

USC CS42

USC R257

ASTM C208

ASTM C532

ASTM C332

ATMA IBI

AIMA IB2

AIMA IB3

AIMA IB4

AIMA IB5

AIMA IB8

ASTM C167

ASTM D2164

USC R78

AAMA 1402.1

AAMA 1403.1
1967

1967

1970

1969

ANSI C33.25

(1969)

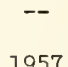

1957

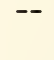

$-$

\section{7 \\ 1968 \\ 1970 \\ IP 1972}

1971

1971

1971

1963

1949

1962

1966

1966

1971

1970

1969

1969

1969

1969

1969

1970

1970

1950

1970

1971
$-$

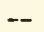

$\because-$

ANSI A52.I

(1969)

-- P

$\begin{array}{ll}-- & P \\ - & 0\end{array}$

-- PO

$--$

$-$

$-$

$-$

$-$

$-$

$-$

$-$

ANSI Z98.8

(1969)

--

$\begin{array}{ll}-- & 0 \\ -- & P \\ -- & 0\end{array}$

UL

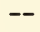

$-$

$=$

-

$-$

SIGMA

--

$--$

$-$

$--$

--

$-$

$-$

--

$\overline{--}$

$-$

$-$

-- 
USC CS31

USC CS199

USC PS1

CRCSHSB

$\subset \mathrm{AA} * 28$

AAMA 62-5-16

AAMA 100

AAMA 64-1

P1805 other doors, including swinging, folding, "track", panel, trap, hatches, etc.

1806 Roofs and roofing materials

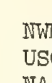

NWMA I.S.I

USC PS38

INAAMM CHM-1

NAAMM *34

FHDA 4

UL 325

USC PS32

USC CSI7I

USC CSI20

AAMA 402.8

AAMA 1303.3

AAMA 1102.5

AAMA 1502

STDI 100

STDI 104

ACI 512

ASTM C222

ASTM C406

ASTM A361

ASTM D224

ASTM D226

ASTM D227

USC R257

UL $55 \mathrm{~A}$

ASTM D371

UL 55B

ASTM D2824

USC R78

ASTM D1227

ASTM D1863

ASTM DII87

ASTM D2626

ASTM D312

ASTM D2822

ASTM D2823

ASTM D 450

ASTM D1327

ASTM DI73
1952

1955

1966

1964

1972-73

1962

1970

1964
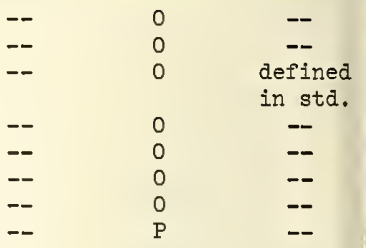

1969

1970

1970

1970

1972

IP 1972

1970

1958

1958

1972

1971

1971

IP 1972

1965

1965

1967

1966

1970

1971

1968

1968

1971

1962

1968

1971

1971

1969

1950

1971

1971

1970

$1969 \mathrm{~T}$

1971

1969

1969

1971

1965

1968

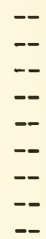

ANSI Al34.2 (1970)

--

ANSI A.134.xx

IP 1972

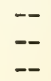

ANSI A.140.I

(1968)

ANSI Al26.1 0

$$
\text { (I967) }
$$

AvSI A109.43

(1971)

ANSI AI09.19

(1969)

ANSI Al09.2 0

(1970)

ANSI A.109.3

(1969)

$\begin{array}{ll}-- & 0 \\ -- & S\end{array}$

ANSI Al09.22 0

(1965)

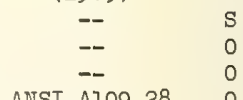

ANSI Al09.28

(1965)

ANSI Al09.27

(1965)

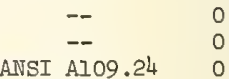

ANSI A109.24

(1965)

$\begin{array}{cc}-- & \text { PO } \\ \text { ANSI AI09.6 } & \text { PO }\end{array}$

$$
\text { (1969) }
$$

ANSI Al09.25 0

(1965)

ANSI A.109.12 0

(1969)
NWMA

- -

$-$

$-$

UL

$-$

$-$

AAMA

A.AMA

AAMA
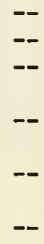

UL

$-$

UL

--

$-$

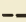

$-$
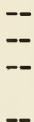

$-$ 


\section{Product Product \\ Number Description}

P1807 Floors and flooring materials

1808 Awnings and shutters, outdoor

1809 Covers, patio and porch

1810 Outside structures, including retaining walls, patios, terraces

1811 Bricks, concrete blocks, not part of structure

\section{AS}

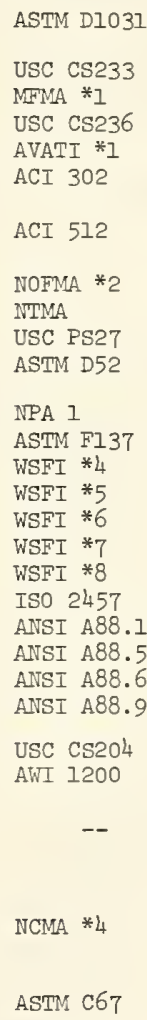

ASTM C62

ASTM CT3

ASTM C126

ASTM C216

ASTM C652

ASTM C426

ANSI Al12.21.2

ANSI Al12.21.1
Latest

Issue or

Status of Standard

Standard

Type of

Certifi-

Accepted by Standard Program

\begin{tabular}{|c|c|c|c|}
\hline 1971 & $\begin{array}{c}\text { ANSI AI09.26 } \\
(1965)\end{array}$ & 0 & -- \\
\hline 1970 & $\begin{array}{l}\text { ANSI A109.4 } \\
(1969)\end{array}$ & 0 & -- \\
\hline 1969 & -- & 0 & -- \\
\hline 1971 & $\begin{array}{c}\text { ANSI A109.20 } \\
\text { (1967) }\end{array}$ & 0 & -- \\
\hline 1968 & $\begin{array}{c}\text { ANSI A109.21 } \\
(1969)\end{array}$ & 0 & -- \\
\hline 1970 & - & $S(T M)$ & -- \\
\hline $105 ?$ & -- & 0 & - \\
\hline & -- & 0 & -- \\
\hline 1970 & -- & $S(T M)$ & -- \\
\hline 1969 & -- & $\mathrm{S}(\mathrm{TM})$ & UL \\
\hline
\end{tabular}

1970

1963

1964

1966

1967

1969

ANSI 010.1

(1960)

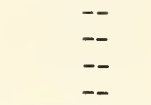

ANSI Al57.I

(1970)

1967

1969

IP 1972

1970

1970

ANSI AI40.I

(1968)

1971

1971

1962

1962

1962

1962

1962

1972

1951-52

1952

1952

1955

1959

1968

--

ANSI 09.I

(1960)

$--$

$-$

$\overline{--}$

$-$

$-$

$--$

$-$

$-$

$-$

$-$

$--$

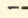

$-$

$-$

$-$

$-$

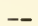

$-$

$-$

$-$

$--$

$-$

$-$

$--$

$-$

$-$

$-$

$-$

--

$-$

$-$

--

--

1948

1966

ANSI A82.1 O(TM)

(1967)

1969

1967

1971

1971

1970

1970

ANSI A98.1

(1970)

ANSI A78.1

(1967)

ANSI Al01.I

(1971)

ANSI A99.1

(1967)

1971

1968

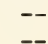

-- 
Product Product

Number Description

P1814 Elevators and other lifts

1815 Windows and window glass, other than storm windows

1816 Scaffolding

1817 Porches, balconies, open side floors and floor openings

1818 Cisterns, cesspools and septic tanks

1819 Nails, carpet tacks and screws, thumbtacks

1820 Hardware, including doorknobs, hinges, cabinet pulls, door springs, etc.

Responsible Organization and Standard Designation ANSI Al7.I and
supplement

USC CS265

USC CS163

SIGMA 65-7-2

ANSI Z97.I

USC C\$266

USC CS264

USC CS205

USC CS204

USC CS190

ASTM E283

ASTM E330

ASTM E33]

ASTM (insulating

glass)

AAMA 302.8

AAMA 1302.3

AAMA 1002.8

AAMA 1502

UL 9

NFPA 257

CSWI

ANSI Al0.8

UL 70

IAPMO PSI

ASTM C380

ASTM C514

ANSI B18.6.1

ANSI B18.6.2

ANSI B18.6.4

ANSI B18.11

ANSI B18.17

ANSI B18.2.1

IFI 171

IFI 104

$\begin{array}{ll}\text { USC CS9 } & 1965 \\ \text { CNAAMM } & 1966 \\ \text { NBHA *69-1 } & 1969 \\ \text { NBHA *65-2 } & 1965 \\ \text { BHMA } 101 & 1969 \\ & \\ \text { BHMA 201 } & 1971 \\ \text { BHMA 301 } & 1971 \\ \text { BHMA 311 } & 1970 \\ \text { BHMA 601 } & 1970 \\ \text { BHMA 1101 } & 1970 \\ \text { BHMA 1201 } & 1970\end{array}$

Latest

Issue or

Status of Standard

Type of

CertifiStandard

Accetted by Standard

cation

Program
1971

1972

1964

1964

1971

1972

1964

1964

1964

1964

1964

1968

1970

1970

IP 1972

1972

1971

1972

IP 1972

1970

1970

1969

1969

1970

1966

1963

1970

1972

1972

1966

1961

1968

1965

1970

1968

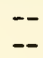

$\mathrm{S}$

S

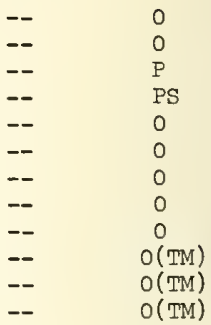$$
\text { (1972) }
$$

$$
\text { ANSI A2.6 }
$$$$
\text { (1968) }
$$
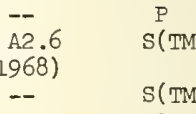

$-$

UL

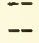

$\mathrm{S}(\mathrm{TM})$

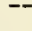

S
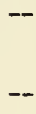

ANSI Al62.I

UL

(1970)

- 0

ISO R272

(1968)

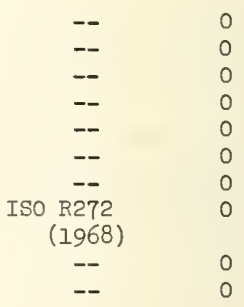

--
--
--
--
--
--
--
--
--
ANSI Al56.1

(1970)

$\begin{array}{ll}-- & 0 \\ -- & 0 \\ -- & 0 \\ -- & 0 \\ -- & 0 \\ -- & 0\end{array}$


BHMA. 401

NBHA *72-1

1971

1972

AAMA 1102.5

ANSI Z97.I

ASTM (insulating

$$
\text { glass) }
$$

CGTA

ANSI 297.1

AAMA 402.8

CGTA

1826 Storm windows

1827 Screen doors

1828 Window screens

1972

1969

1972

1972

1969

1972

1972

1964

1955

1968

1964
AAMA 1002.8

FHDA 4

USC CS248

USC CS138

SCRMA SMS-2003

USC $\operatorname{Csl} 63$

USC CS240

USC CS241

USC CS248

USC CSl38

SCRMA SMR-1003

CSWA V.I

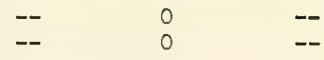

1971 ANSI Al34.xx SP IP 1972

IP 1972
ANSI AI34.2 (1972)
$--$

ANSI A]34.3

(1972)

1961

1961

1964

1961

1968

1970

$\begin{array}{ll}-- & 0 \\ -- & 0 \\ -- & 0 \\ -- & 0\end{array}$

$-$

$\overline{-z}$

$-$

$-$

$-$

1829 Handrails, railings and banisters

1830 Separate garages, tool sheds, dog houses, and other pet enclosures

1831 Window sills, door sills, door frames, window frames
USC CS266

USC CS265

USC CS264

USC CS205

USC CS204

USC CS190

USC CSl63

USC CS208

UL 9
1964

1964

1964

1964

1964

1964

1964

1957

1970

$\begin{array}{ccc}-- & 0 & -- \\ -- & 0 & -- \\ -- & 0 & -- \\ -- & 0 & -- \\ -- & 0 & -- \\ -- & 0 & -- \\ -- & 0 & -- \\ -- & 0 & -- \\ \text { ANSI A2.6 } & \text { S(TM) } & \text { UL }\end{array}$

AAMA

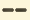

GTA $\overline{A A M A}$ GTA AAMA

$\vdots$
$\vdots$
$\vdots$
$\vdots$
-

UL
1832 Wire, not electric, including picture hanging wire, barbed wire, constmaction wire

1833 Other construction materials
CSWA V.I

AIMA *6

AIMA IB7 AAMA 701.1
1970

1967

1971

1965 
Product Product

Number Description

P1834 Fences, not electric, outdoor, all types, including posts

1835 Jalousie glass doors

1836 Jalousie glass windows

1837 Glass door, not otherwise specified

1838 Lumber, boards, paneling pieces, not part of structure

1839 Plaster
Issue or

Status of Standard Type of cation Standard
Certifi-

cype of cation
USC PS20

AWPA C1

AWPB *1

HPMA S-69

USC PS1

WWPA *23

NHLA *I

NHIA *2

ISO 768

ISO 769

USC $\operatorname{Cs} 236$

USC CS176

USC PS51

USC CS251

$\begin{array}{lc}\text { USC CS246 } & 1962 \\ \text { USC CS269 } & 1965 \\ \text { ASTM } & \text { IP } 1972 \\ \text { CLFMI *65-1 } & 1965 \\ \text { CLFMI *65-3 } & 1965\end{array}$

\section{CLFMI}

CIFMI
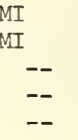

1972
ANSI Al34.I (1972)

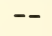

1970

1972

1971

1969

(interim)

1966

1970

1971

1965

1972

1972

1966

1958

(revision IP)

1971

1963

ASTM C28

ASTM C587

ASTM C207

ASTM C6
1968

1968

1968

1968

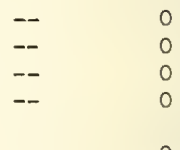

$-\infty \quad 0$

$-$

$-$

$-$

$-$

$-$

--

$-$

ANSI A49.3 0

(1970)

ANSI K67.13

(1969)

ANSI K67.2

(1969)
CLFMI

CLFMI

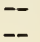

--

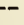

AAMA

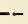

$-$

AWPB

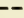

defined in std.

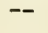

$--$

$-$

$-$

$-$

$-$

$-$

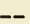

$-$ 
In process (IP) tities found under the column "Latest Issue or Status of standard" are not included in this listing.

Only titles found under the column "Responsible Organization and Standard Designation," will be found in this listing.

\begin{tabular}{|c|c|}
\hline$A A$ & $* 28$ \\
\hline AAMA & $62-5-16$ \\
\hline AAMA & $64-1$ \\
\hline AAMA & 100 \\
\hline AAMA & 302.8 \\
\hline AAMA & 402.8 \\
\hline AAMA & 701.1 \\
\hline AAMA & 1002.8 \\
\hline AAMA & 1102.5 \\
\hline AAMA & 1302.3 \\
\hline AAMA & 1303.3 \\
\hline AAMA & 1402.1 \\
\hline AAMA & 1403.1 \\
\hline ABFLO & $J-1$ \\
\hline ABFLO & $J-2$ \\
\hline ABFLO & $\mathrm{J}-3$ \\
\hline ABFLO & $J-4$ \\
\hline$A B Y C$ & \\
\hline $\mathrm{ACI}$ & 302 \\
\hline $\mathrm{ACI}$ & 512 \\
\hline AFTMA & ${ }^{*} 1$ \\
\hline AHAM & $\mathrm{CN}-1$ \\
\hline AHAM & $\mathrm{DA}-1$ \\
\hline AHAM & $\mathrm{DA}-2$ \\
\hline AHAM & $\mathrm{DA}-3$ \\
\hline AHAM & $D A-4$ \\
\hline AHAM & $\mathrm{DA}-5$ \\
\hline AHAM & DA-6 \\
\hline AHAM & $\mathrm{DA}-7$ \\
\hline AHAM & $\mathrm{DA}-8$ \\
\hline AHAM & $\mathrm{DA}-9$ \\
\hline AHAM & $D A-10$ \\
\hline AHAM & $\mathrm{DH}-1$ \\
\hline AHAM & ER-1 \\
\hline AHAM & HLW-1 \\
\hline AHAM & HRF-I \\
\hline AHAM & $\mathrm{HS}-1$ \\
\hline AHAM & HU-1 \\
\hline AHAM & RAC-1 \\
\hline AHAM & RAC-2SR \\
\hline AIMA & IBI \\
\hline AIMA & IB2 \\
\hline AIMA & IB3 \\
\hline AIMA & IB 4 \\
\hline ATMA & IB5 \\
\hline AIMA & IBT \\
\hline AIMA & IB \\
\hline AIMA & *6 \\
\hline AMCA & 21 \\
\hline AMO & *1 \\
\hline
\end{tabular}

Aluminum Standards and Data

Specification and Methods of Test for Aluminum Residential Siding

Thermal Resistance of Aluminum Siding

Aluminum Siding Specification

Specifications for Aluminum Prime Windows

Specifications for Aluminum Sliding Glass Doors

Specification for Woven Pile Weatherstrip

Specifications for Aluminum Combination Vertically-Siding or Horizontally-

Operating Storm Windows for External Application

Specifications for Aluminum Combination Storm Doors

Standards for Forced-Entry Resistant Aluminum Horizontal Sliding Windows

Standards for Forced-Entry Resistant Aluminum Sliding Glass Doors

Specifications for Aluminum Siding

Specification for Installation of Residential Aluminum Siding

Method of Measuring the Finished Size of Bed Pillows

Method of Measurement for Determining Length of Bed Pillows

Method of Measurement for Determining Width of Bed Pillows

Method of Measuring the Length and Width of Mattress Pads

Safety Standards for Small Craft; Pleasure Boats, Engines, Boat Trailers, Marine Products

Recommended Practice for Concrete Floor and Slab Construction

Recommended Practice for Manufactured Reinforced Concrete Floor and Roof Units

New Fly Line Standards (Fishing Tackle)

AHAM Room Air Conditioner Standard

Electric Housewares; Household Automatic Electric Dry and Steam Irons Standard

AHAM Electrically Heated Bed Coverings Standard

AHAM Electric Waffle Baker and Sandwich Grill Standard

AHAM Electric Coffeemaker Standard

AHAM Electric Housewares; Hot Plates and Disc Stoves Standard

Electric Housewares; Household Portable Fan-Forced and Radiant Electric Air Heaters Standard

Electric Housewares; Household Automatic Electric Toasters Standard

Electric Housewares; Household Flectric Food Blenders Standard

Electric Housewares; Household Automatic Electric Fry Pans, Griddles, Saucepans, Dutch Ovens, and Deep Fat Fryers Standard

Electric Housewares; Household Electric Can Openers Standard

Dehumidifier Standard

Household Electric Ranges Standard

Household Washer Performance Evaluation Procedure Standard

AHAM Refrigerator and Freezer Standard

Dishwasher Standard

Appliance Humidifier Standard

Room Air Conditioner Sound Rating Standard

Room Air Conditioner Standard

Recommended Product and Application Specification Structural Insulation Roof Deck

Recommended Product and Application Specification 1/2-inch Fiberboard Nail-Base

Sheathing

Recommended Product and Application Specification 1/2-inch Intermediate

Fiberboard Sheathing

Product Specification for Sound Deadening Board in Wall Assemblies

Product Specification for Non-Combustible Sound Deadening Board in Wall

Assemblies

Test Procedures for Washablility and Scrubbability of Finishes Applied to Tiles and Panels

Recommended Product and Application Specification Insulation Board Underlayment

for Laminated Wood Block Flooring

Recommended Product and Application Specification for Fiberboard Ceiling Tile

Test Code for Air Moving Devices

Bow, String Length and Wood Arrow Standards 
AIISI AIO 0.8

ANSI AI2.I

ANSI AI 4.I

ANSI AI 4.2

ANSI AI7.I

ANSI A88.1

ANSI A88.2

ANSI A88.3

ANSI A88.4

ANSI A88.5

ANSI A88.6

ANSI A88.9

ANSI AI12.21.1

ANSI AIl2.21.2

ANSI AI61.I.

ANSI B18.2.1

ANSI B18.6.1

ANSI B18,6.2

ANSI BI8.6.4

ANSI B18.11

ANSI B18.17

ANSI B30.1

ANSI B71.1

ANSI B94.2

ANSI B94.11

ANSI B94.12

ANSI B121.1

ANSI C12

ANSI C18.1

ANSI $\mathrm{C} 37.7$

ANSI $\quad 037.14$

ANSI $\quad 37.15$

ANSI C62.2

ANSI C70.I.

ANSI C72.1

ANSI C73

ANSI C78

ANSI C78.1.05

ANSI L22

ANSI L22.30.2

ANSI L22.30.4

ANSI L22.30.6

ANSI L22.30.7

ANSI L22.30.8

ANSI L22.30.9

ANSI L22.30.10

ANSI L22.30.11

ANSI L22.30.15

ANSI I27.1

ANSI MH2

ANSI PHI.14

ANSI PHI.2I

ANSI PHI.25

ANSI PHI.26

ANSI PHI.29

ANSI PHI. 31

ANSI PHI. 37

ANSI PH3.15

ANSI PH3.27
Safety Requirements for Scaffolding

Safety Requirements for Floor and Wall Openings, Railings and Toe Boards

Safety Code for Portable Wood Ladders

Safety Code for Portable Metal Ladders

Safety Code for Elevators, Dumbwaiters, Escalators, and Moving Walks

Specification for Preparation of Subfloors to Receive Oxychloride Composition

Flooring

Specification for General Purpose Oxychloride Composition Flooring and Its Installation

Specification for Heavy Duty Oxychloride Composition Flooring and Its Installation Specification for Basecoat Oxychloride Composition Flooring and Its Installation Specification for Non-Slip Oxychloride Composition Flooring and Its Installation Specification for Terrazzo Oxychloride Composition Flooring and Its Installation Specification for Nonspark Conductive Oxychloride Composition Flooring and Its Installation

Standard for Floor Drains

Standard for Roof Drains

Minimum Construction and Performance Standards for Kitchen Cabinets

Standard for Square and Hex Bolts and Screws

Standard for Slotted and Recessed Head Wood Screws

Standard for Hexagon Head Cap Screws, Slotted Head Cap Screws, Square Head Set Screws, and Slotted Headless Set Screws

Standard for Slotted and Recessed Head Tapping Screws and Metallic Drive Screws Standard for Miniature Screws

Standard for Wing Nuts, Thumb Screws, and Wing Screws

Safety Code for Jacks

Safety Specifications for Power Lawn Mowers

Standard for Reamers

Standard for Straight Shank and Taper Shank Combined Drills and Countersinks Standara for Carbide - Tipped Masonry Drills and Blanks for Carbide - Tipped Masonry Drills

Standard Specifications for Hack Saw Blades

Code for Electricity Metering

Standard for Dry Cells and Batteries

Standard for Interrupting Rating Factors for Reclosing Service for AC High-Voltage Circuit Breakers Rated on a Total Current Basis

Standard for Low-Voltage Direct-Current Power Circuit Breakers and Anode Circuit Breakers

Standard for Rated Control Voltages and Their Ranges for Low-Voltage Air Circuit Breakers

Standard Guide for Application of Valve-Type Lightning Arresters for AlternatingCurrent Systems

Standard for Household Automatic Electric Flatirons

Standard for Household Automatic Electric Storage-Type Water Heaters

Standards on Plugs and Receptacles, C73 Series

Standard Dimensional and Electrical Characteristics of Electric Discharge Lamps

(Fluorescent)

Standard for Spotlight and Floodlight Service 115, 120, and 125 Volts

Standard Performance Requirements for Textile Fabrics

Standard Performence Requirements for Beth Mat and Throw-Rug Fabrics

Standard Performance Requirements for Woven Household Blanket Fabrics

Standard Performance Requirements for Woven Drapery Fabrics

Standard Performance Requirements for Knitted Sheet Fabrics (Tricot)

Standard Performance Requirements for Woven Pillowcase and Sheet Fabrics

Standard Performance Requirements for Woven Shower Curtain Fabrics

Standard Performance Requirements for Woven Slipcover Fabrics

Standard Performance Requirements for Woven Napery and Tablecloth Fabrics

Standard Performance Requirements for Woven Window Curtain Fabrics

Standard for Nomenclature for Bedding Sizes

Standard Specifications for Metal Drums and Pails

Standard Dimensions for $35 \mathrm{~mm}$ Film Magazines and Film for Still Picture Cameras

Standard Dimensions for Roll Film, Becking Paper, and Spools

Standard Specifications for Safety Photographic Film

Standard Dimensions for Film Packs

Standard Methods for Determining the Curl of Photographic Film

Standard Method for Determining the Brittleness of Photographic Film

Stendard Methods for Determining the Scratch Resistance of Processed Photographic Film

Specifications for Printing Frames

Specifications for Lantern Slide Projectors 
ANSI PH3.28

ANSI PH3. 39

ANSI PH3. 49

ANSI PH4.100 thru

PH 4.304

ANSI PH4.33

ANSI PH4.125

ANSI PH22.5

ANSI PH22.12

ANSI PH22.109

ANSI PH22.110

ANSI 53.3

ANSI 53.8

ANSI $\mathrm{X} 4.4$

ANSI Z21.1.1

ANSI $\mathrm{Z} 21.1 .2$

ANSI $\mathrm{Z} 21.5 .1$

ANSI Z21.6

ANSI Z21.9

ANSI Z21.10.1

AIVSI Z21.10.3

AISI $\mathrm{z} 21.11 .1$

ANST $\mathrm{Z} 21.11 .2$

ANSI $\mathrm{Z21.13}$

ANSI Z21.16

ANISI Z21.19

AIVSI Z21.40.1

AIISI Z21.40.2

ANSI Z21.42

ANSI Z21. 44

AIISI Z21.47

AINSI Z21.48

AlNSI Z21. 49

ANSI Z21.52

AIISI Z21.53

ANSI Z21.55

ANSI Z21.57

ANSI $\mathrm{Z} 24.22$

ANSI $\mathrm{Z} 37.17$

ANSI $\mathrm{z} 41.1$

ANSI Z49.1

ANSI 261.1

ANSI $\quad$ Z66.1

ANSI $\mathrm{Z} 80.1$

ANSI $\mathrm{Z} 80.2$

ANSI 287.1

ANSI $\mathrm{Z} 88.2$

ANSI $\mathrm{Z} 90.1$

ANSI $\mathrm{Z} 91.1$

ANS $\mathrm{Z} 97.1$

ANSI Z124.1

AIVSI Z124.2

Specifications for Slidefilm Projectors

Standard for Picture Sizes for Roll Film, 35m Film, and 126 Film Still-Picture Cameras

Standard for General-Purpose Photographic Exposure Meters (Photoelectric Type)

Specifications for Photographic Grade Chemicals

Stardard Method for the Determination of Silver in Photographic Films, Papers, Fixing Baths, or Residues

Standard Specification for Photographic Grade p-Methylaminophenol Sulfate

Standard Dimensions for $16 \mathrm{~mm}$ Motion-Picture Film, 2R-3000

Standard Dimensions for $16 \mathrm{~mm}$ Motion-Picture Film, 1R-3000

Standard Dimensions for $16 \mathrm{~m}$ Motion-Picture Film, IR-2994

Standard Dimensions for $16 \mathrm{~mm}$ Motion-Picture Film, 2R-2994

Method for Measurement of Electroacoustical Characteristics of Hearing Aids

Method for Expressing Hearing Aid Performance

Basic Sheet Sizes and Standard Stock Sizes for Bond Papers and Index Bristols

Standard for Domestic Gas Ranges - Free Standing Units, Volume 1, Including

Addendas Z2.1.1a-1968 and Z21.1.1b-1969

Standard for Domestic Gas Ranges - Built-In Domestic Cooking Units, Volume II, Including Addendes $\mathrm{Z} 21.1 .2 \mathrm{a}$ and $\mathrm{Z} 21.1 .2 \mathrm{~b}$

Standard for Gas Clothes Dryers, Volume 1, Type 1 Clothes Dryers, Including

Addenda Z21.5.1a.

Standard for Domestic Gas-Fired Incinerators

Approval Requirements for Domestic Hot Plates and Laundry Stoves

Standard for Gas Water Heater, Volume 1, Automatic Storage Type Water Heaters with Inputs of 75,000 BTU per hour or less

Standard for Approval Requirements for Gas Water Heaters, Volume III Circulating

Tank, Instantaneous and Large Automatic Storage Type Water Heaters

Standard for Approval Requirements for Gas-Fired Room Heaters, Volume 1, Vented

Room Heaters

Standard for Gas-Fired Room Heaters Volume II, Unvented Room Heaters

Standard for Gas-Fired Steam and Hot Water Boilers, Including Addendas z2l.13a

and $\mathrm{Z} 21.13 \mathrm{~b}$

Standard for Gas Unit Heaters, Including Addendas z2l.16a and z21.16b

Standard for Approval Requirements for Refrigerators Using Gas Fuel

Standard for Gas-Fired Absorption Sumer Air Conditioning Appliances, Including

Addenda Z21.40.1a

Standard for Gas Engine-Powered Summer Air Conditioning Appliances

Standard for Approval Requirements for Gas-Fired Illuminating Appliances

Standard for Approvel Requirements for Gas-Fired Gravity and Fan Type Sealed

Combustion System Wall Furnaces

Standard for Gas-Fired Gravity and Forced Air Central Furnaces

Standard for Gas-Fired Gravity and Fan Type Floor Furnaces, Including Addendas $\mathrm{Z} 21.48 \mathrm{a}$ and $\mathrm{Z} 21.48 \mathrm{~b}$

Standard for Gas-Fired Gravity and Fan Type Vented Wall Furnaces, Including

Addendas Z21.49a and $\mathrm{Z} 21.49 \mathrm{~b}$

Standard for Gas-Fired Single Firebox Boilers, Including Addendas Z21.52a and $\mathrm{z} 21.52 \mathrm{~b}$

Standard for Gas-Fired Heavy Duty Forced Air Heaters

Standard for Gas-Fired Sauna Heaters

Recreational Vehicle Cooking Gas Appliances

Standard Method for Measurement of the Real-Ear Attenuation of Ear Protectors at

Threshold

Standard for Acceptable Concentrations of Carbon Tetrachloride

Standard for Men's Safety-Toe Footwear

Standard for Safety in Welding and Cutting

Standard Dimensions, Tolerances, and Terminology for Home Cooking and Baking

Utensils

Specifications for Paints and Coatings Accessible to Children, to Minimize Dry Film Toxicity

Standard Requirements for First-Quality Prescription 0phtha.7mic Lenses

Standard for Prescription Requirements for First-Quality Contact Lenses

Practice for Occupational and Educational Eye and Face Protection

Practices for Respiratory Protection

Specifications for Protective Headgear for Vehicular Users

Standard for Performance Requirements for 0il-Powered Centrol Furnaces

Performance Specifications and Methods of Test for Transparent Safety Glazing

Material Used in Buildings

Standard for Gel-Coated Glass-Fiber Reinforced Polyester Resin Bathtub Units

Standard for Gel-Coated Glass-Fiber Reinforced Polyester Resin Shower Receptor and Shower Stall Units 
Official Methods of Analysis of the Association of Official Agricultural

Chemists

ARI 210

ARI 230

ARI 240

ARI 280

ARI 310

ARI 443

ARI 610

ARI 620

ARI 630

ARI 1010

ASHRAE 13

ASHRAE 15

ASHRAE 18

ASME PCTI7

ASSE 1006

ASSE 1007

ASSE 1008

ASTM A53

ASTM A72

ASTM A74

ASTM A120

ASTM A268

ASTM A312

ASTM A361

ASTM A651

ASTM B42

ASTM B43

ASTM B75

ASTM B88

ASTM B135

ASTM B251

ASTM B302

ASTM B306

ASTM B315

ASTM B543

ASTM C6

ASTM C28

ASTM C61

ASTM C62

ASTM C67

ASTM C73

ASTM C9I

ASTM C94

ASTM C126

ASTM Cl47

ASTM C148

ASTM C149

ASTM C167

ASTM C206

ASTM C207

ASTM C208

ASTM C216

ASTM C222

ASTM C225

ASTM C327

Standard for Unitary Air-Conditioning Equipment

Standard for Application of Year-Round Residential Air Conditioning

Standard for Unitary Heat Pump Equipment

Standard for Central Forced-Air Electric Heating Equipment

Standard for Packaged Terminal Air-Conditioners

Standard for Sound Rating of Room Fan-Coil Air-Conditioners

Standard for Central System Humidifiers

Standard for Portable and Self-Contained Humidifiers

Standard for Selection, Installation and Servicing of Humidifiers

Standard for Drinking-Fountains and Self-Contained, Mechanically-Refrigerated

Drinking-Water Coolers

Methods of Testing for Household Refrigerators, Combination Refrigerator-Freezers, and Household Freezers

Safety Code for Mechanical Refrigeration

Methods of Testing for Rating Drinking-Water Coolers with Self-Contained Mechanical Refrigeration Systems

Power Test Codes for Internal-Combustion Engines

Performance Requirements for Household Dishwashers

Performance Requirements for Home Laundry Equipment

Performance Requirements for Household Food Waste Disposer Units

Specification for Welded and Seamless Steel Pipe

Specification for Welded Wrought-Iron Pipe

Specification for Cast Iron Soil Pipe and Fittings

Specification for Black and Hot-Dipped Zinc-Coated (Galvanized) Welded and

Seamless Steel Pipe for Ordinary Uses

Specification for Seamless and Welded Ferritic Stainless Steel Tubing for

General Service

Specification for Seamless and Welded Austenitic Stainless steel Pipe

Standard Specification for Steel Sheet, Zinc Coated (Galvanized) by the Hot-Dip

Process for Roofing

Specification for Stainless Steel Water-DWV Tube

Specifications for Seamless Copper Pipe, Standard Sizes

Specification for Seamless Red Brass Pipe, Standard Sizes

Specification for Seamless Copper Tube

Specifications for Seamless Copper Water Tube

Specification for Seamless Brass Tube

Specification for General Requirements for Wrought Seamless Copper and Copper-Alloy Tube

Specification for Threadless Copper Pipe

Specifications for Copper Drainage Tube

Standard Specification for Copper-Silicon Alloy Seamless Pipe and Tube

Specification for Welded Copper and Copper Alloy Tube

Standard Specification for Normal Finishing Hydrated Lime

Specification for Gypsum Plasters

Standard Specification for Keene's Cement

Standard Specification for Building Brick (Solid Masonry Units Made From Clay or Shale)

Standard Methods of Sampling and Testing Brick

Standard Specification for Calcium Silicate Face Brick (Sand-Lime Brick)

Standard Specification for Masonry Cement

Standard Specification for Ready-Mixed Concrete

Standard Specification for Ceramic Glazed Structural Clay Facing Tile, Facing

Brick, and Solid Masonry Units

Standard Methods of Internal Pressure Test on Glass Containers

Standard Methods of Polariscopic Examination of Glass Containers

Standard Method of Thermal Shock Test on Glass Containers

Standard Methods of Test for Thickness and Density of Blanket or Batt-Type Thermal

Insulating Materials

Standard Specification for Special Finishing Hydrated Lime

Standard Specification for Hydrated Lime for Masonry Purposes

Standard Specification for Structural Insulating Board Made from Vegetable Fibers

Standard Specification for Facing Brick (Solid Masonry Units Made from Clay or

Shale)

Standard Specification for Asbestos-Cement Roofing Shingles

Standard Method of Test for Resistance of Glass Containers to Chemical Attack Standard Method of Test for Linear Thermal Expansion of Fired Ceramic Whiteware Materials by the Interferometric Method 
Standard Specification for Lightweight Aggregates for Insulating Concrete Standard Method of Test for Impact Resistance of Ceramic Tableware Standard Method of Test for Moisture Expansion of Fired Whiteware Products Standard Method of Test for Linear Thermal Expansion of Fired Whiteware Products by the Dilatometer Method

Standard Specification for Annular Ringed Nails for Gypsum Wallboard

Standard Specification for Aggregates for Masonry Grout

Standard Specification for Roofing Slate

Standard Method of Test for Compressive (Crushing) Strength of Fired Whiteware Materials

Standard Method of Test for Thermal Conductivity of Whiteware Ceramics

Standard Method of Test for Crazing Resistance of Fired Glazed Whitewares by

Autoclave Treatment

Standard Method of Test for Drying Shrinkage of Concrete Block

Standard Specification for Mortar and Grout for Reinforced Masonry

Standard Specification for Chemical Admixtures for Concrete

Standard Specification for Nails for the Application of Gypsum Wallboard

Standard Specification for Structural Insulating Formboard Made from Vegetable

Fibers

Specification for Slate Blackboards

Standard Method of Test for Crazing Resistance of Fired Glazed Ceramic

Whitewares by a Thermal Shock Method

Standard Method of Test for Resistance of Overglaze Decorations to Attack by

Detergents

Standard Specification for Adhesives for Fastening Gypsum Wallboard to Wood Framing

Specification for Gypsum Veneer Plaster

Standard Specification for Hollow Brick (Hollow Masonry Units Made from Clay or Shale)

Standard Methods for Flexural Properties of Ceramic Whiteware Materials

Standard Methods of Test for Detergent Resistance of Ceramic Decorations on Glass Tableware

Standard Specification for Spirits of Turpentine

Standard Specification for Wood Paving Blocks for Exposed Platforms, Pavements, Driveways, and Interior Floors Exposed to Wet and Dry Conditions

Standard Specification for Friction Tape for General Use for Electrical Purposes Standard Specification for Rubber Insulating Tape

Standard Method of Analysis of Lubricating Grease

Standard Method of Testing Varnishes

Standard Specification for Woven Cotton Fabries Saturated with Bituminous

Substances for Use in Waterproofing

Standard Method of Test for Burning Quality of Kerosene

Standard Method of Test for Cone Penetration of Iubricating Grease

Standard Specification for Asphalt Roll Roofing Surfaced with Powdered Talc and Mica

Standard Specification for Asphalt Shingles Surfaced with Mineral Granules

Standard Specification for Asphalt-Saturated Roofing Felt for Use in

Waterproofing and in Constructing Built-Up Roofs

Standard Specification for Coal-Tar Saturated Roofing Felt for Use in

Waterproofing and in Constructing Built-Up Roofs

Sampling and Testing for Turpentine

Standard Specifcation for Asphalt Roll Roofing Surfaced with Mineral Granules

Standard Specification for Asphalt-Saturated Asbestos Felts for Use in

Waterproofing and in Constructing Built-Up Roofs

Tests for Impact Resistance of Plastics and Electrical Insulating Materials

Standard Methods of Testing and Tolerances for Woven Tapes

Standard Methods of Testing Varnished Cotton Fabrics and Varnished Cotton Fabric

Tapes Used for Electrical Insulation

Standard Specification for Asphalt for Use in Constructing Built-Up Roof

Coverings

Standard Specification and Tolerances for Woven Asbestos Tapes

Standard Method of Testing Clear Lacquers and Lacquer Enamels

Standard Specification for Woven Cotton Tapes for Electric Purposes

Standard Specifications for Shellac Varnishes

Standard Specification for Wide Selvage Asphalt Roll Roofing Surfaced with

Mineral Granules

Standard Methods of Testing for Woven and Tufted Pile Floor Coverings

Standard Specification for Gasoline

Standard Specification for Coal-Tar Pitch for Roofing, Dampproofing, and Waterproofing 
ASTM D 455

ASTM D 456

ASTM D496

ASTM D497

ASTM D498

ASTM D499

ASTM D526

ASTM D533

ASTM D534

ASTM D580

ASTM D593

ASTM D630

ASTM D639

ASTM D690

ASTM D691

ASTM D71 4

ASTM D738

ASTM D739

ASTM D770

ASTM D773

ASTM D799

ASTM D802

ASTM D816

ASTM D897

ASTM D929

ASTM D930

ASTM D950

ASTM D1000

ASTM DI031

ASTM D1084

ASTM Dllll

ASTM DI112

ASTM D1116

ASTM D1119

ASTM D1120

ASTM D1121

ASTM DI122

ASTM DI123

ASTM DI124

ASTM D1151

ASTM D1177

ASTM DI187

ASTM D1227

ASTM D1230

ASTM D1233

ASTM D1279

ASTM D1281

ASTM D1287

ASTM D1327

ASTM D1335

ASTM D1361

ASTM D1367

ASTM D1374

ASTM D1384

Standard Specification for Milled Toilet Soap

Standard Specification for Caustic Soda (Anhydrous)

Standard Specification for Chip Soap

Standard Specification for Ordinary Laundry Bar Soap

Standard Specification for Powdered Soap (Nonalkaline Soap Powder)

Standard Specification for White Floating Toilet Soap

Standard Methods of Test for Lead in Gasoline, Gravimetric Method

Standard Specification for Built Soap, Powdered

Standard Specification for Alkaline Soap Powder

Standard Methods of Testing and Tolerances for Woven Glass Tapes

Standard Specification for Salt-Water Soap (Coconut Oil Type)

Standard Specification for Olive Oil Chip Soap (Type A, Straight; Type B

Blended)

Standard Methods of Testing for Battery Containers Made from Hard Rubber or

Equivalent Materials

Standard Specification for Compound Chip Soap (with Rosin)

Standard Specification for Compound Powdered Soap (Granulated with Rosin)

Standard Method of Evaluating Degree of Blistering of Paints

Testing and Tolerances for Rope made from Bast and Leaf Fibers

Tests and Tolerances for Spun, Twisted, or Braided Products Made From Flax, Hemp, Ramie or Mixtures Thereof

Standard Specification for Isopropyl Alcohol (Isopropanol)

Standard Method of Test for Adhesiveness of Gumed Tape

Standard Specification for Liquid Toilet Soap

Standard Methods of Sampling and Testing of Pine $0 i l$

Standard Methods of Testing for Rubber Cements

Standard Method of Test for Tensile Properties of Adhesive Bonds

Standard Specification for Borax

Total Immersion Corrosion Test of Water-Soluble Aluminum Cleaners

Standard Method of Test for Impact Strength of Adhesive Bonds

Standard Methods of Testing for Pressure-Sensitive Adhesive Coated Tapes Used

for Electrical Insulation

Standard Specification for Creosoted End-Grain Wood Block Flooring for Interior Use

Standard Methods of Tests for Viscosity of Adhesives

Standard Specification for Chip or Granular Soap for Low-Temperature Washing

(Low and Medium Titer)

Standard Specification for Solid Soap for Low Temperature Washing (Low and Medium Titer)

Standard Method of Test for Resistance of Pile Floor Coverings to Attack by Black Carpet Beetle Larvae

Standard Method of Test for Ash Content of Engine Antifreezes and Antirusts

Standard Method of Test for Boiling Point of Engine Antifreezes

Standard Method of Test for Reserve Alkalinity of Engine Antifreezes and

Antirusts

Standard Method of Test for Specific Gravity ô Engine Antifreezes by the Hydrometer

Standard Method of Test for Water in Concentrated Engine Antifreezes by the Iodine Reagent Method

Standard Specification for Hydrometer-Thermometer Field Tester for Engine Antifreezes

Standard Method of Test for Effect of Moisture and Temperature on Adhesive Bonds Standard Method of Test for Freezing Point of Aqueous Engine Antifreeze Solution Standard Specification for Asphalt-Base Enulsions for Use as Protective Coatings for Metal

Standard Specification for Asphalt-Base Emulsions for Use as Protective Coatings for Built-Up Roofs

Standard Method of Test for Flammability of Clothing Textiles

Standard Methods of Testing Twine Made From Bast and Leaf Fibers

Standard Method of Test for Buffering Action of Metal Cleaners

Standard Method of Test for Rinsing Properties of Metal Cleaners

Standard Method of Test for $\mathrm{pH}$ of Engine Antifreezes and Antirusts

Standard Specification for Woven Burlap Fabrics Saturated with Bituminous

Substances for Use in Waterproofing

Standard Method of Test for Tuft Bind of Pile Floor Coverings

Standard Methods of Test for Fire Retardancy of Paints (Stick and Wick Method)

Standard Method of Test for Lubricating Qualities of Graphites

Aerated Total Immersion Corrosion Test for Metal Cleaners

Standard Method for Corrosion Test for Engine Antifreezes in Glassware 
ASTM D1436

AS่TM D1437

ASTM DI527

ASTM D1567

ASTM D1630

ASTM D1668

ASTM D1709

ASTM D1762

ASTM D1779

ASTM D1785

ASTM D1792

ASTM D1793

ASTM DI817

ASTM D1862

ASTM D1863

ASTM D1880

ASTM D1881

ASTM DI882

ASTM D1916

ASTM D2047

ASTM D2088

ASTM D2095

ASTM D2103

ASTM D2104

ASTM D2141

ASTM D2148

ASTM D2164

ASTM D2178

ASTM D2179

ASTM D2202

ASTM D2203

ASTM D2206

ASTM D2239

ASTM D224I

ASTM D2265

ASTM D2266

ASTM D2282

ASTM D2301

ASTM D2322

ASTM D2348

ASTM D2349

ASTM D2356

ASTM D2366

ASTM D2369

ASTM D2377

ASTM D2401

Standard Methods for Application of Emulsion Floor Polishes to Substrates for Testing Purposes

Standard Method of Test for Flash Point of Solvent-Type Liquia Waxes

Specification for Acrylonitrile-Butadiene-Styrene (ABS) Plastic Pipe, Schedules 40 and 80

Standard Methods of Testing for Detergent Cleaners for Evaluation of Corrosive Effects on Certain Porcelain Enamels

Standard Method of Test for Abrasion Resistance of Rubber Compounds for Soles and Heels

Standard Specification for Woven Glass Fabrics Treated with Bituminous Substances for Use in Waterproofing

Standard Method of Test for Impact Resistance of Polyethylene Film by the Free Falling Dart Method

Chemical Analysis of Wood Charcoal

Specification for Adhesive for Acoustical Materials

Specifications for Poly(Vinyl Chloride) (PVC) Plastic Pipe, Schedules 40, 80, and 120

Standard Method of Test for Potential Long-Term Removability Properties of Conventional Type Water-Fmulsion Floor Polishes

Standard Method of Test for Water Spotting of Emulsion Floor Polishes

Standard Method of Test for Specific Gravity of Rubber Chemicals

Specification for Laminated-Wall Bituminized Fiber Drain and Sewer Pipe

Standard Specification for Mineral Aggregate for Use on Built-Up Roofs

Recommended Practice for Selection of Engine Antifreezes for Use in Automotive Cooling Systems, Ethylene Glycol, and Methanol Types

Standard Method of Test for Foaming Tendencies of Engine Antifreezes in Glassware Standard Method of Test for Effect of Antifreeze and Cooling System.Chemical

Solutions on Organic Finishes for Automatic Vehicles

Standard Method of Test for Penetration of Adhesives

Standard Method of Test for Static Coefficient of Friction of Polish-Coated

Floor Surface as Measured by the James Machine

Standard Method for Determination of Low Concentrations of Lead in Paint

Standard Method of Test for Tensile Strength of Adhesives by Means of Bar and Rod Specimens

Standard Specification for Polyethylene Film and Sheeting

Specifications for Polyethylene (PE) Plastic Pipe, Schedule 40

Standard Method of Test for Adhesion Ratio of Polyethylene Film

Standard Methods of Testing Bondable Silicone Rubber Tapes Used for Electrical

Insulation

Standard Methods of Test for Structural Insulating Roof Deck

Standard Specification for Asphalt-Impregnated Glass Fiber Mat (Felt)

Standard Specification for Combination Bar Soap

Standard Method of Test for Slump of Oil and Resin-Base Caulking Compounds

Standard Method of Test for Staining of $0 i l$ and Resin-Base Caulking Compounds

Standard Method of Test for Determination of Low Quantities of Mercury in Paint

Standard Specification for Polyethylene (PE) Plastic Pipe (SDR-PR)

Standard Specification for Poly(Vinyl Chloride) (PVC) Plastic Pipe (SDR-PR and

Class T)

Standard Method of Test for Dropping Point of Lubricating Grease of Wide

Temperature Range

Standard Method of Test for Wear Preventive Characteristics of Lubricating

Grease (Four-Ball Method)

Standard Specification for Acrylonitrile-Butadiene-Styrene (ABS) Plastic Pipe

(SDR-PR and Class T)

Standard Specification for Vinyl Chloride Plastic Pressure-Sensitive Electrical

Insulating Tape

Standard Method of Test for Resistance of Chrome-Tanned White Shoe Upper Leather

to Artificial Perspiration

Standard Method of Test for Arsenic in Paint

Standard Method of Qualitative Determination of Nature of Thinner in Solvent Type

\section{Paints}

Standard Specification for Scouring Powder Compounds

Accelerated Testing of Moisture Blister Resistance of Exterior House Paints on

Wood

Standara Methods of Test for Volatile Content of Paints

Standard Method of Test for Tack-Free Time of 0 il and Resin-Base Caulking Compounds Standard Method of Test for Service Change of Appearance of Pile Floor Coverings 
Standard Specifications for Polyethylene (PE) Plastic Pipe, Schedules 40 and 80 Based on Outside Diameter

ASTM D2450

ASTM D2452

ASTM D2453

ASTM D2463

ASTM D2484

ASTM D2486

ASTM D2509

ASTM D2513

ASTM D2533

ASTM D2559

ASTM D2561

ASTM D2570

ASTM D2571

ASTM D2578

ASTM D2595

ASTM D2625

ASTM D2626

ASTM D2646

ASTM D2659

ASTM D266I

ASTM D2665

ASTM D2672

ASTM D2677

ASTM D2684

ASTM D2686

ASTM D2754

ASTM D2758

ASTM D2762

ASTM D2763

ASTM D2782

ASTM D2783

ASTM D2815

ASTM D2822

ASTM D2823

ASTM D2824

ASTM D2832

ASTM D2834

ASTM D2847

ASTM D2851

ASTM D2859

ASTM D2860

ASTM D2869

ASTM D2931

Standard Method of Test for Bond of Oil and Resin-Base Caulking Compound Standard Method of Test for Extrudability of $0 i l$ and Resin-Base Caulking Compounds

Standard Method of Test for Shrinkage and Tenacity of $0 i l$ and Resin-Base Cauzking Compounds

Standard Method of Test for Drop Impact Resistance of Polyethylene Blow-Molded Containers

Standard Specification for Polyester Film Pressure-Sensitive Electrical

Insulating Tape

Standard Methods of Test for Scrub Resistance of Interior Latex Flat Wall Paints

Standard Method of Test for Measurement of Extreme Pressure Properties of

Lubricating Grease (Timken Method)

Standard Specification for Thermoplastic Gas Pressure Pipe, Tubing, and Fittings

Standard Method of Test for Vapor-Liquid Ratio of Gasoline

Standard Specification for Adhesives for Structural Laminated Wood Products for

Use Under Exterior (Wet Use) Exposure Conditions

Standard Method of Test for Environmental Stress Crack Resistnace of Blow-Molded Polyethylene Containers

Standard Method for Simulated Service Corrosion Testing of Engine Antifreezes

Recommended Practice for Testing of Wood Furniture Lacquers

Standard Method of Test for Wetting Tension of Polyethylene and Polypropylene Films

Standard Method of Test for Evaporation Loss of Lubricating Greases Over

Wide-Temperature Range

Standard Method for Determining Endurance (Wear) Life and Load-Carrying Capacity of Dry Solid Film Lubricants

Standard Specification for Asphalt-Base Sheet for Use in Construction of Built-Up Roofs

Standard Methods of Testing for Backing Fabrics

Standard Method of Test for Column Crush Properties of Blown Thermoplastic

Containers

Specification for Acrylonitrile-Butadiene-Styrene (ABS) Plastic Drain, Waste, and Vent Pipe and Fittings

Specification for Poly(Vinyl Chloride) (PVC) Plastic Drain, Waste and Vent Pipe and Fittings

Standard Specification for Bell-End Poly(Vinyl Chloride) (PVC) Pipe

Standard Method of Test for Lightability of Barbecue Briquets

Recommended Practice for Determining Permeability of Thermoplastic Containers

Standard Specification for Tetrafluórethylene-Backed Pressure-Sensitive Electrical

Insulating Tape

Standard Specification for High-Temperature Glass Cloth Pressure-Sensitive

Electrical Tape

Standard Methods of Testing Engine Antifreezes by Engine Dynamometer

Standard Specification for Drycleaning Detergent, Non-Charge Type

Standard Specification for Drycleaning Detergent, Liquid Type

Standard Method for Measurement of Extreme-Pressure Properties of Lubricating Fluids (Timken Method)

Standard Method for Measurement of Extreme-Pressure Properties of Lubricating Fluids (Four-Ball Method)

Standard Specification for General Purpose Cleaning Compound

Standard Specification for Asphalt Roof Cement

Standard Specification for Asphalt Roof Coatings

Standard Specification for Asphalt-Based, Aluminum Roof Coatings

Recommended Practice for Determining Nonvolatile Content of Paint and Paint

Materials

Standard Method of Test for Nonvolatile Matter (Total Solids) in Water Frnulsion

Waxes, Solvent Based Waxes and Polymer Fmulsion Floor Waxes

Recommended Practice for Testing of Engine Coolants in Vehicle Service

Standard Specification for Liquid Optical Adhesive

Standard Method of Test for Flammability of Finished Textile Floor Covering

Materials

Standard Method of Test for Adhesion of Pressure-Sensitive Tape to Fiberboard at 90 Degree Angle and Constant Stress

Standard Method of Test for Black Heel Marking Tendencies of Waxes and Polymer Floor Finishes

Recommended Practices for Testing Latex Flat Wall Paints 
Recommended Practices for Testing Exterior Solvent-Thinned House and Trim Paints

ASTM D2955

ASTM D2957

ASTM D2958

ASTM D2971

ASTM D3061

ASTM D3068

ASTM D3069

ASTM D3071

ASTM D3072

ASTM D 3074

ASTM D3075

ASTM D3088

ASTM D3089

ASTM D3092

ASTM D3093

ASTM D3094

ASTM D3095

ASTM D3096

ASTM E108

ASTM E283

ASTM E291

ASTM E330

ASTM E331

ASTM E345

ASTM F137

AVATI *1

AVATI *3

AVATI $* 4$

AVATI $* 5$

AVATI *6

AVATI *15

AWI 800

AWI 1200

AWPA $\mathrm{Cl}$

AWPB *1

AWWA $B 501$

AWWA C700

AWWA C706

AWWA E101

$\mathrm{BCA} * 1$

BCI *1.15

BCI *1.41

BCI *1. 48

BHMA 101

BHMA 201

BHMA 301

BHMA 311

BHMA 401

BHMA 501

BHMA 601

BHMA 1101

BHMA 1201

BIA EM

BIA HEM

BMA 6

BMA $6 \mathrm{~T}$

Standard Specification for Hand Scrubbing Soap (Grit Soap) Paste and Bar

Standard Specification for Hand Scrubbing Soap (Grit Soap) Powder

Standard Specification for Powdered Toilet Soap

Standard Method of Test for Stitch-Quilted Products

Standard Specification for Tin-Plate Fabricated Aerosol Cans

Standard Specification for Safe Fill for Aerosols

Standard Method of Test for Delivery Rate of Aerosol Products

Standard Method of Drop Testing of Glass Aerosol Bottles

Standard Methods of Test for Volatile Content of Aerosol Products

Standard Methods of Test for Pressure in Metal Aerosol Containers

Standard Methods of Test for Overrun of Food Aerosols

Standard Test for Effectiveness of Aerosol and Pressuriz ed Space Spray

Insecticides Against Flying Insects

Standard Method of Measurement of the A-D Dimension of Aerosol Valve Dip Tubes

Standard Test for Particle Size Distribution of Space Insecticide Aerosol

Standard Test for Pickup Efficiency of Residual Aerosol Insecticides

Standard Test for Seepage Rate of Aerosol Products

Standard Test for Effectiveness of Aerosol Insecticides Against Cockroaches

Standard Test for Liquid Densities of Pressurized Products

Standard Methods of Fire Tests of Roof Coverings

Standard Method of Test for Rate of Air Leakage Through Windows

Chemical Analysis of Caustic Soda and Caustic Potash (Sodium Hydroxide and

Potassium Hydroxide)

Standard Method of Test for Structural Performance of Exterior Windows, Curtain

Walls, and Doors Under the Influence of Wind Loads

Standard Method of Test for Water Penetration of Exterior Windows, Curtain

Walls, and Doors by Uniform Static Air Pressure Differential

Standard Method of Tension Testing of Metallic Foil

Standard Method of Test for Flexibility of Resilient Flooring Materials with

Cylindrical Mandrel Apparatus

Specification for Asphalt Floor Tile

Specification for Asphalt Tile Adhesive Cut-Back Type

Specification for Water Emulsion Type Adhesive for Asphalt and Vinyl Asbestos

Tile

Specification for Cleaners for Use on Asphalt and Vinyl Asbestos Tile Floors

Specification for Water Emulsion Floor Polish for Use on Asphalt or Vinyl Asbestos

Tile Floors

Specification for Asphalt and Vinyl Asbestos Tile Adhesive

Standard for Stairwork

Standard for Blinds and Shutters

All Timber Products - Preservative Treatment by Pressure Processes

Quality Mark Lumber, Timber and Plywood

Standard for Caustic Soda

Standard for Cold-Water Meters-Displacement Type

Standard for Direct Reading Remote Registration Systems for Cold-Water Meters

Standard for Deep Well. Vertical Turbine Pumps-Line Shaft and Submersible Types

Sanctioned Tournament Table and Accessory Equipment Specifications

Recommended Domestic Marketing Standards for the Storage Manufacturing Industry

(in Their Yearbook)

Battery Materials Specifications for the Storage Manufacturing Industry (in Their Yearbook)

Battery Council International Storage Battery Specifications for the Storage

Manufacturing Industry (in Their Yearbook)

Butts and Hinges Standard

Cabinet Hardware Standard

Door Controls (Closers) Standard

Door Controls (Overhead Holderś) Standard

Sliding and Folding Door Hardware Standard

Auxiliary Locks Standard

Locks and Lock Trim Standard

Spring Hinges Standard

Auxiliary Hardware Standard

Recommended Practices for Pleasure Boats, Engines, Boat Trailers, and Marine

Products

Houseboat Engineering Manual

Safety Standards for Regular Bicycles

Test Procecures for Regular Bicycles 
CAGI *I

CCTI B-2ll.2

$\operatorname{CCTI} B-211.3$

CCTI $B-211.4$

CCTI 8401.3

2

7

10

10, pt.1

10, pt.2, sect.E Specification for Clocks

10, pt.2, sect.J Specification for Washing Machines

10, pt.2, sect.K Specification for Spin Extractors

10, pt.2, sect.N Specification for Shavers, Hair Clippers and Similar Appliances

10, pt.2, sect.0 Specification for Massage Appliances

Il, pt.I

Specification for Electric Cooking and Heating Appliances for Domestic and Similar Purposes

CEE

CEE

CEE

$\mathrm{CEE}$

CEE

$\mathrm{CEE}$

$\mathrm{CEE}$

CEE

$\mathrm{CEE}$

$\mathrm{CEE}$

$C E E$

CEE

11, pt.2, sect.A Particular Specification for Cooking Ranges, Cooling Tables and Similar Appliances

11, pt.2, sect.B Particular Specification for Appliances for Heating Iiquids

11, pt.2, sect.C Particular Specification for Portable Immersion Heaters

11, pt.2, sect.E Particular Specification for Stationary Non-Instantaneous Water Heaters

11, pt.2, sect.F Particular Specification for Room Heating and Similar Appliances

11, pt.2, sect.G Particular Specification for Electric Irons, Ironers and Pressing Machines

11, pt.2, sect.M Particular Specification for Waffle Irons, Grills, Warming Plates and Other Dry Cooking Appliances

11, pt.2, sect.N Particular Specification for Clothes Dryers and Towel Dryers

13

14

16

18

19

20

24

CI $\%$

CLFMI *65-1

CIFMI *65-3

CMI *1-11

CSMA AG

CSMA Bul.12

CSMA Bul.41

CSMA Bul.46

CSMA Bul.60

CSMA Bul.81

CSMA Bul.117

CSMA Bul.180

CSMA Bul.198

CSMA Bul.245

CSMA Bul.335

CTFA 4

EEI MS J-10

EIA RECI33

EIA RECI4I

EIA RS2IIC

EIA SEIO3

FHDA 4

FMS $6051 \& 6052$

GA $\quad * 17$

GTA
Specification for Polyvinyl Chloride Insulated Cables and Flexible Cords

Specification for Switches for Domestic and Similar General Purposes

Specification for D-Type Fuses for Domestic and Similar General Purposes

Specification for Voltage-Operated Earth-Leakage Circuit-Breakers for Domestic and Similar Purposes

Specification for Miniature Circuit-Breakers for Domestic and Similar Purposes Specification for Portable Motor-Operated Tools

Specification for Switches for Appliances

Specifications for Synthetic Ropes

Standard Test Methods for Stranded and Plaited Synthetic Fiber Ropes

Specifications for Residential Steel Chain Link Fence

Industrial Steel Specifications for Fence-Posts, Gates and Accessories

Recommended Standards for Sanitary Can Dimensions

Aerosol Guide

CSMA Procedure for Products Classified as to Slip Resistant

Foam Estimation of Iiquid Detergents for Hand Dishwashing

Method for Furniture Polish Evaluation

Tentative Test Method for Determining Freeze-Thaw Resist a nee of Iiquid Polishes

Suggested Practices for Traffic Testing Floor Polishes Under Non-Laboratory

Controlled Conditions

Tentative Test Method for Qualitative Determination of Slip Resistance of Floor Polishes

Estimation of Foam in Mechanical Dishwashing Detergents

Tentative Overglaze Removal Test, Dishwashing (Mechanical) Detergent Solutions

Tentative Test Methods for Waxes, Polishes and Floor Finishes

Bench Test Method for Soil Resistance for Floor Finishes

Standard for Borax

Standards for Watthour Meters

Standard Magnetic Recorder Combined with Home Radio Receivers

Standard for VHF Recelving Antenna Performance, Presentation and Measurement

EIA Standard Dimensional Characteristics of Monophonic and Stereophonic Disc

Phonograph Records for Home Use 78 R.P.M., 45 R.P.M., $33-1 / 3$ R.P.M.

Standard Speakers for Sound Equipment

Product Standard for Douglas Fir, Western Hemlock, and Sitka Spruce Doors and Blinds

Approval Standard for Safety Containers and Filling and Supply Containers

Requirements for Gypsum Wallboard in Bath Shower Areas

Engineering Standards Manual 1969 
HAIC *I

HPMA S-69

IAPMO PSI

IAPMO PS2-66

IAPMO PST

IAPMO PSII

IAPMO PSI 4

ISPMO PSI7

IAPMO. PSI 8

IAPMO PS23

IAPMO PS24

IAPMO PS27

IAPMO PS28

IAPMO PS29

IAPMO TSCI

IAPMO TSC2

IAPMO TSC4

IAPMO TSC 8

IAPMO TSC9

IAPMO TSCII

IAPMO TSC12

IAPMO TSC13

IAPMO TSCI 4

IAPMO TSC15

IAPMO TSCl6

IAPMO TSCl 8

IAPMO TSC19

IEC 43

IEC 65

IEC 86-1

IEC 86-2

IEC $86-3$

IEC 94

IEC $94 \mathrm{~A}$

IEC 99-1

IEC 170

IEC 174

IEC 175

IEC 176

IEC $227 \mathrm{~A}$

IEC 268-3

IEC 299

IEC 311

IEC $\quad 312$

IEC 335-1

IEC 335-2

IEC 335-3

IEC 335-4

IEC $335-5$

Interim Bone Conduction Thresholds for Audiometry

Interim Industry Standard for Hardwood Plywood

Prefabricated Concrete Septic Tanks

Cast Brass and Tubing P-Traps

Tubing Trap Wall Adapters

Material and Property Standard for Prefabricated Shower Receptors, Shower

Enclosures, and Non-Metallic Bathtubs

Material and Property Standard for Flexible Copper Water Connectors

Supplemental Standard to Standard Specification for Acrylonitrile-Butadiene-

Styrene (ABS)

Material and Property Standard for Cultured Marble Lavatory

Material and Property Standard for Dishwasher Drain Airgaps

Material and Property Standard for Polyethylene Pipe for Cold Water Service and Yard Piping

Supplemental Standard to Specification for Poly(Vinyl Chloride) (PVC)

Material and Property Standard for Welded Red Brass (Copper Alloy 230) Water Tube Material and Property Standard for Welded Red Brass (Copper Alloy 230) Drainage Tube

Standard for Mechanical Seal Toilets for Use in Travel Trailers and Camp Cars and Self-Propelled Vehicles

Standard for Waste Holding Tanks

Standard for Pressurized and Non-Pressurized Portable Water Tanks

Material and Property Standard for Backflow Preventors and Relief valves

Standard for Gas Supply Connectors for Mobile Homes

Standard for Non-Metallic Shower Receptors, Stall Showers, Lavatories, Bathtubs, and Combination Tank and Shower Receptor Units

Trailer Standard for Self-Contained Electrically Operated Recirculating,

Chemically Controlled Toilet

Standard for Water Pressure Caps for Use in Travel Trailers, Camp Cars and

Trailer Coaches

Standard for Electric Pressure Pumps for Use in Recreational Vehicles

Standard for Hand Actuated Non-Pressure Water Pumps

Standard for ABS Lavatories for Recreational Vehicles

Standard for Fiberglass Kitchen Sinks

Standard for Flexible Non-Pressure Water Fill Hose

Recommendations for Alternating Current Watthour Meters

Recommendation for Safety Requirements for Mains Operated Electronic and Related

Equipment for Domestic and Similar General Use

Recommendation for Primary Cells and Batteries (Part 1: General)

Recommendation for Primary Cells and Batteries (Part 3: Terminals)

Recommendation for Primary Cells and Batteries (Part 2: Specification Sheets)

Recommendation for Magnetic Tape Recording and Reproducing Systems: Dimensions and Characteristics

Recommendation for Cassette for Commercial Tape Records and Domestic Use

Dimensions and Characteristics (Supplement 1)

Recommendation for Lightning Arresters; Non-Linear Resistor Type Arresters for

A.C. Systems

Recomendation for Class 1,0 Alternating-Current Watthour Meters

Recommendation for A.C. Electric Pedestal Type Fans and Regulators

Recommendation for A.C. Electric Table Type Fans and Regulators

Recommendation for A.C. Flectric Ceiling Type Fans and Regulators

Recommendation for Polyvinyl Chloride Insulated Flexible Cables and Cords with

Circular Conductors and a Rated Voltage not Exceeding $750 \mathrm{~V}$ Single-Core Cable for

Internal Wiring of Household Appliances (Supplement 1)

Recommendation for Sound System Equipment, Part 3 Sound System Amplifiers

Recommendation for Measurement of the Performance Characteristics of Electric

Blankets

Recommendation for Methods of Measuremert of Performance of Electric Irons for

Household or Similar Use

Recommendation for Methods of Measurement of Performance of Vacuum Cleaners for

Household Use

Recommendation for Safety of Household and Similar Electrical Appliances, Part

1: General Requirements

Recommendation for Safety of Household and Similar Electrical Appliances, Part

2: Particular Requirements for Vacuum Cleaners

Recommendation for Safety of Household and Similar Electrical Appliances; Part

3: Particular Requirements for Electric Irons

Recommendation for Safety of Household and Similar Electrical Appliances; Part

4: Particular Requirements for Spin Extractors

Recommendation for Safety of Household and Similar Electrical Appliances; Part

5: Particular Requirements for Dishwashers 
Recommendation for Safety of Household and Similar Electrical Appliances; Part 6: Particular Requirements for Ranges

Recomendation for Safety of Household and Similar Electrical Appliances; Part 7: Particular Requirements for Washing Machines

Recommendation for Safety of Household and Similar Electrical Appliances; Part 8: Particular Requirements for Electric Shavers, Hair Clippers and Similar Appliances

9: Particular Requirements for Toasters, Grills, Waffle Irons and Roasters Recommendation for Safety Requirements for Electric Fans and Regulators Recommendation for Methods for Measuring Performance of Floor Polishers for Household and Similar Purposes

Standard for Surge Arresters (Lightning Arresters) for Alternating-Current Power Circuits

Standara for Neutral Grounding Devices

Test Procedure for Single-Phase Induction Motors

IEEE Test Procedure for Frequency-Modulated Mobile Communications Receivers

IEEE Standard Method for Measurement of Weighted Peak Flutter of Sound Recording and Reproducing Equipment

Standard Method for Measuring Transmission Performance of Telephone Sets Standard Methods of Measuring Recorded Flux of Magnetic Sound Records at Medium Wavelengths

Mechanical and Quality Requirements for Stainless Steel and Nonferrous Bolts, Screws, Studs and Nuts

Hex Flange Screws

IHF Standard Methods of Measurement for Audio Amplifiers

Incinerator Standards

Specification for Shellac

Recommendation for Hermetically Sealed Metal Food Containers

Recommendation for Pipes of Plastics Materials for the Transport of Fluids (Outside Diameters and Nominal Pressures)

Recommendation for Files and Rasps Lengths and Cross Sections Recommendation for Parallel Shank Twist Drills Jobber and Stub Series and Morse Taper Shank Twist Drills

Recommendation for Hand Reamers and Iong Fluted Machine Reamers Morse Taper Shank

Recommendation for Lighting and Signalling for Motor Vehicles and Trailers

Recommendation for Gymnastic Equipment - Parallel Bars

Recommendation for Gymnastic Equipment - Horizontal Bars

Recommendation for Gymnastic Equipment - Rings

Recommendation for Gymnastic Equipment - Vaulting Horse and Pommelled Horse

Recommendation for Gymnastic Equipment - Balancing Beam

Recommendation for Gum Spirit of Turpentine and Wood Turpentines

Recommendation for Specification for Photographic Grade Sodium Sulphite

Recommendation for Specification for Photographic Grade Sodium Triosulphate, Crystalline

Recommendation for Specification for Photographic Grade Potassium Bromide

Recommendation for Method for Indicating the Stability of the Images of Processed Black and White Films, Plates and Papers

Recommendation for Specification for Photographic Grade Hyaroquinone

Recommendation for Specification for Photographic Grade Sodium Carbonate, Anhydrous Recommendation for Lens Aperture Markings for Still Cameras Recommendation for Dimensions of Small Flash Connections for Hand-Held Cameras Recommendation for Determination of the Thickness of Single Sheets of Paper Recommendation for Steel Pipes for Gas, Water and Sewage, Welded or Seamless Recommendation for Syringes for Medical Use Recommendation for Hypodermic Needles

Recommendation for $35 \mathrm{~mm}$ Filmstrips - Dimensions and Formats Recomendation for Dimensions for 127,120 and 620 Roll Film, Backing Paper and Film Spools

Standard for Fibre Building Boards - Determination of Ben ding Strength Standard for Fibre Building Boards - Hard and Medium Boards - Determination of Water Absorption and of Swelling in Thickness After Immersion in Water Recommendation for Household Refrigerators; Part 1: Performance Requirements Recommendation for Household Refrigerators; Part 2: Compartments for the Storage of Frozen Foodstuffs

Recommendation for Testing and Rating Room Air Conditioners

Recommendation for Dimensions for $35 \mathrm{~mm}$ Film Magazines for Still Picture Use and Test for Film Pullout Force 


\section{NAAMM *33 \\ NAAMM * 34 \\ NAAMM CHMI \\ NAFM *1 \\ NAPF C-1-70 \\ INAR *I \\ NBHA *65-2 \\ NBHA *69-1 \\ NBHA *72-1 \\ NCMA $* 4$ \\ NCMA *11}

\section{NCRPM *1}

NEMA DC3

NEMA DC15

NEMA EI2O

NEMMA EW-1

NEMA FLI

NEMA HE2

NEMA LAI

NEMA MGI

NEMA MG2

NEMA RI2

NEMA SB2

Recommendation for Finishes with External Screw Thread for Glass Containers and Gauges for the Inspection of Screw Closures

International Standard for Photography - Expendable Photoflash Lamps -

Determination of the Light Output

Recommendation for Determination of Volatile and Non-Volatile Matter

Recommendation for Paints and Varnishes - Determination of the Danger

Classification by Flashpoint (Closed Cup Method)

Recommendation for Paints and Varnishes - Surface-Drying Test (Ballontini Method)

Recommendation for Paints and Varnishes - Scratch Test

Recommendation for Paints and Varnishes - Bend Test (Cylindrical Mandrel)

Recommendation for Paints and Varnishes - Cupping Test

Recommendation for Paints and Varnishes - Determination of Resistance to Water -

Water Immersion Method

Recommendation for Paints and Varnishes - Determination of Flashpoint (Closed Cup Method)

Recommendation for Paints and Varnishes - Determination of Fineness of Grind Recommendation for Hand Operated Wrenches and Sockets

Recommendation for Carpets; Determination of Mass of Pile Per Unit Area That Can Be Shorn Away From the Backing

International Standard for Water-Resistant Watches, Part l: Wristwatches - Water Resistance for General Use

International Standard for Hand and Machine Hacksaw Blades - Dimensions for Lengths Up to $450 \mathrm{~mm}$ and Pitches up to $6.3 \mathrm{~mm}$

International Standard for Screwdriver Bits, For Slotted Head Screws, with Male Hexagon Drive

International Standard for Screwdriver Blades for Slotted Head Screws

International Standard for Paints and Varnishes - Cross-Cut Test

International Standard for Paints and Varnishes - Determination of Flow Time By Use of a Flow Cup

International Standard for Classification of Solid Beech Parquet Strips

International Standard for Hand-Knotted Carpets - Determination of Tuft Leg Length Above the Woven Ground

International Standard for Hand-Made Carpets - Determination of Types of Knots

International Standard for Machine-Made Textile Floor Coverings - Determination of Dimensional Changes in Varying Moisture Conditions

Engineering Standard for Net Load Recommendations for He a ting Boilers, Baseboard, Finned-Tube

Standard Specifications Official Grading Rules for Hard Maple Flooring

Specifications for Motorcycle Safety Helmets

Safety Code for Portable Metal and Fiberglass Ladders

Hardware Standards Contained in: The National Association of Architectural Metal

Manufacturers Entrance Manual

Specification for Metal Stairs Manual

Standard for Fire-Rated Custom Metal Doors and Frames

Specifications for Custom Hollow Metal Doors and Frames

Specifications, Standards, and Test Procedures for Upholstered Furniture Fabrics

Product Standard for Plastic Surfaced Vanity Cabinets

U.S. Model Rocket Sporting Code

Hardware for Labelled Fire Doors

Recommendations Concerning Hardware for Aluminum Doors

Hardware Reinforcements on Steel Doors and Frames

Recommendations for Reinforced Concrete Masonry Retaining Walls

Notes and Typical Details on the Use of Concrete Masonry Small Swimming Pool

Construction (Suggested Specifications)

$\mathrm{X}$-Ray Protection Standards for Home Television Receivers

Standards Publication for Automatic Temperature Controls - Room Thermostats

Standards Publication for Line-Voltage Room Thermostats

Standards for Watthour Meters

Standards Publication for Electric Arc-Welding Apparatus

Standards Publication for Floodlights

Standard for Self-Contained Electric Comfort Heaters

Standards Publication for Lightning Arresters

Standards Publication for Motors and Generators

Standards Publication for Motor and Generators (Safety Standard for Construction and Guide for Selection, Installation and Use of Fractional- and IntegralHorsepower Motors and Generators)

Standards Publication for General-Purpose and Communication Battery Chargers Standards Publication for Signalling Apparatus; Electrically-Operated Home-Fire Alarm Systems 
NFPA 101

NFPA 211

NFPA 224

NFPA 256

INPA 257

INPA 302

NFPA $501 B$

NFPA 501C

NHLA *I

NHLA *2

NLGI

NMRA

NOFMA *2

NPA 1

NPBA *1

NSDA *1

NSF 14

NSF 17

NSF 19

NSF 27

NSF 28

NSF 31

NSF 32

NSF 38

NSF 39

NSPI *2

NTMA 17

NTMA 18

NWMA I.S.I

PI $3 p 54$

PI $\quad 110.2$

PI $\quad 110.6$

PI 110.7

PI $\quad 110.8$

PI 110.11

PI 110.12

PI 110.13

PI $0-2 r$

PI T3608p

PI T3609p

PI T3610p

PI T3612p

PI T3614p

PI T3615p

PI T3618p

PI T3619p

PI T3620p

PI T3627p

PI T3632s
Standards Publication for Low-Voltage Power Circuit Breakers

Standards Publication for Alternating-Current General-Use Snap Switches

Standards Publication for Special-Purpose Wiring Devices

Maintenance and Use of Portable Fire Extinguishers

Code for Model Rocketry

National Electrical Code

ArSI Standard Electrical Code for One- and Two-Family Dwellings

Standard for the Installation, Maintenance and Use of a Household Fire Warning System

Lightring Protection Code

Incinerators and Rubbish Handing

Life Safety Code

Chimneys, Fireplaces, and Venting systems

Homes and Camps in Forest Areas

Fire Tests of Roof Coverings

Standard for Fire Tests of Window Assemblies

Standard for Motor Craft (Pleasure and Commercial)

Standard for Mobile Homes; Body and Frame Design and Construction Requirements

Standard for Recreation Vehicles; Travel Trailers, Camping Trailers, Truck Campers, Motor Homes

Rules for the Measurement and Inspection of Hardwood and Cypress Lumber

Rules for the Measurement and Inspection of Veneers and Thin Lumber

American Standard for Classification of Lubricating Grease

Standards and Recommended Practices Contained In: National Model Railroad

Association Bulletin (Special Issue) 1971

Specification Manual for Certified Oak Floors: Standard Oak Flooring Grades

Standard for Particleboard for Mobile Home Decking

Box Covering Paper Specifications

Bottle Specifications Guideline

Thermoplastic Materials-Pipe, Fittings, Valves, Joining Materials

National Swiming Pool Institute and National Sanitation Foundation Joint Standard Relating to Centrifugal Pumps for Swimming Pools

National Swiming Pool Institute and National Sanitation Foundation Joint Standard Relating to Adjustable Output Rate Chemical Feeding Equipment for Swimming Pools

Standard for Multiport Valves for Swimming Pools

Standard for Cartridge and High Permeability Type Filters for Swimming Pools

Standard for Polyethylene Refuse Bags

Standard for Paper Refuse Sacks

Standard for Test Kits for Swimming Pool Water

Standard for Resilient Artificial Recreational Surfaces (For Use Around Swimming Pools)

Suggested Minimum Standards for Residential Swimming Pools

Specification for Cast in Place Terrazzo Stairs

Specification for Precast Terrazzo Stairs

Industry Standard for Hardwood Veneered Including Hardboard and Plastic Faced

Flush Doors

Method of Testing for Resistance of Ink on Printed Packaging Materials to Fats and Oils in the Package

Amount of Paraffin on Cartons - Quality of Coverage (Method)

Water Absorption of Paperboard (Method)

Carton Score (Resistance to) Bend and Score Bend Springback (Method)

Wax Absorption of Paper and Carton Board (Method)

Glue Setting Time for Paperboard (Method)

Carton (Resistance to) Opening Force and Opening Force Springback (Method)

Carton Board Stiffness and Carton Board Springback (Method)

Recommended Specification for Closure Sizes and Gages (\#400, 410, 415, 425)

Standard Test Procedure for Tensile Breaking Strength of Paper and Paperboard

Standard Test Procedure for Stretch of Paper and Paperboard

Standard Test Procedure for Rigidity, Stiffness and Softness of Paper and

Paperboard

Standard Test Procedure for Brightness of Paper and Paperboard

Standard Test Procedure for Edge Tearing Resistance of Paper

Standard Test Procedure for Wet Tensile Breaking Strength of Paper and Paperboard

Standard Test Procedure for Compression Resistance of Paperboard (Ring Crush Test)

Standard Test Procedure for Bending Quality of Paperboard

Standard Test Procedure for Water Resistance of Paper and Paperboard

Standard Test Procedure for Moldability (Crease Retention) of Wrapping Paper

Suggested Test Procedure for Roughness or Smoothness of Flat Kraft Paper for Multiwall Bags 
Standard Test Procedure for Puncture Test of Container Board Guidelines and Standards for the Folding Paper Box Industry Test Methods for Pressure Sensitive Tapes

Recomended Standards and Specifications for Tablets and Related Products Certigrade Handbook of Red Cedar Shingles

Standard for Recording and Reproducing Characteristics

Standards for Stereophonic Disc Records

Dimensional Standards for Disc Phonograph Records for Home Use

Standards for Magnetic Tape Records

RMA Handbook for Hose

Standard for Motor Vehicle Seat Belt Assemblies

Standard for Automotive Rubber Mats

Recommended Practice for Engine Oil Performance and Engine Service Classification Recommended Practice for Exterior Sound Level for Snowmobiles

Recommended Practice for Life Test for Automotive Storage Batteries

Storage Battery Standards

Standard for Grounding of Storage Batteries

Standard for Six and Twelve Volt Cigar Lighter Receptacles

Recommended Practice for Semiautomatic Headlamp Beam Switching Devices

Standard for Fog Lamps

Standard for Electric Emergency Lanterns

Standard for Liquid Burning Emergency Flares

Recommended Practice for Test Procedure and Ratings for Hot Water Heaters for Motor Vehicles

Standard for Passenger Car Automatic Transmission Test Code

Standard for Safety Motor Vehicle Seat Belt Anchorage

Recommended Practice for Passenger Car Windshield Defrosting Systems

Recommended Practice for Passenger Car Windshield Wiper Systems

Recomended Practice for Manual Control Sequence for Automatic Transmissions

Recommended Practice for Passenger Car Windshield Washer Systems

Standard for Motor Vehicle Brake Fluid

Specifications for Aluminum Tubuler Frame Screens for Windows

Specifications for Aluminum Sliding Screen Doors

Specification for Sealed Insulating Glass Units

National Warm Air Heating and Air Conditioning Code

Low Velocity Duct Construction Standards

Fibrous Glass Duct Construction Standards

Standard for Protective Headgear

Recommended Practice for Plastic Bottle Visual Color Evaluation

Dimensions for Plastic Bottles

SPI Column Crush Test

Test for Drop Impact Resistance of Plastic Containers

Method of Test for Plastic Bottle Permeability and Compatibility of Packaged Products

Recommended Practice for Evaluating Surface Treatment of Polyethylene Containers Using the Water Dip, Ink Adhesion and Label Adhesion Tests

Method of Test for Closure Torque on Plastic Containers

Performence Requirements for High Density Polyethylene Plastic Bottles

Domestic Sump Pump Standards

Recommended Specification for Standard Steel Doors and Frames

Recommended Specifications for Steel Bi-Fold Closet Doors and Frames

Standards Manual Volume I - Round Wire

Standards Manual Volume II - Flat and Shaped Wires

Recommended Specifications for Steel Windows

Standard for Fiber Analysis of Paper and Paperboard

Standard for Tensile Breaking Strength of Paper and Paperboard

Standard for Weight per Unit Area (Basis Weight or Substance) of Paper and

Paperboard

Standard for Thickness (Caliper) of Paper and Paperboard

Standard for Water Resistance of Paper and Paperboard (Dry-Indicator Method)

Standard for Brightness of Paper and Paperboard

Standard for Stretch of Paper and Paperboard

Standard for Flame Resistance of Treated Paper and Paperboard

Standard for Blood Resistance of Butchers' Wrapping Paper

Standard for Stiffness of Paperboard

Standard for Water Absorption of Paperboard

Standard for Tensile Breaking Properties of Paper and Paperboard (Using Constant

Rate of Elongation Apparatus)

Standard for Bending Number of Paperboard

Standard for Surface Wax on Waxed Paper or Paperboard 
TAPPI T510

TAPPI T516

TAPPI T613

TAPPI T807

TAPPI T808

TAPPI T809

TAPPI T810

UL 7

UL 8

UL 9

UL 20

UL 25

UL 30

UL 41

UL 44

UL 45

UL 50

UL 55A

UL $55 \mathrm{~B}$

UL 57

UL 62

UL 65

UL 69

UL 70

UL 73

UL 83

UL 103

UL 105

UL 112

UL 114

UL 115

UL 123

UL 127

UL 130

UL 133

UL 136

UL 141

UL 153

UL 154

UL 168

UL 174

UL 181

UL 184

UL 198

UL 198.3

UL 250

UL 299

UL $307 \mathrm{~A}$

UL $\quad 307 \mathrm{~B}$

$\begin{array}{ll}\text { UL } & 311 \\ \text { UL } & 399 \\ \text { UL } & 414 \\ \text { UL } & 430 \\ \text { UL } & 452 \\ \text { UL } & 464 \\ \text { UL } & 465 \\ \text { UL } & 467 \\ \text { UL } & 474 \\ \text { UL } & 482 \\ \text { UL } & 484\end{array}$

Standard for Softness of Sanitary Tissues

Standard for Internal Bond Strength of Paper and Paperboard as Measured by a Z-Directional Tensile Test

Standard for Water Resistance of Adhesive Bond in Laminated Paper and Paperboard Standard for Envelope Seal, Seam, and Window Patch Testing

Standard for Analysis of Caustic Soda.

Standard for Bursting Strength of Paperboard and Linerboard

Standard for Flat Crush Test of Corrugated Board

Standard for Flat Crush of Corrugating Medium

Standard for Bursting Strength of Corrugated and Solid Fiberboard

Standard for Safety for Soda-Acid Fire Extinguishers

Standard for Safety for Foam Fire Extinguishers

Standard for Safety for Fire Tests of Window Assemblies

Standard for Safety for Snap Switches

Standard for Safety for Meters for Flammable Liquids and LP-Gas

Standard for Safety for Metal Safety Cans

Standard for Safety for Fire Hazard Classification of Sweeping Compounds

Standard for Safety for Rubber-Insulated Wires and Cables

Standard for Safety for Electric Tools

Standard for Safety for Cabinets and Boxes

Standard for Safety for Materials for Use in Construction of Built-Up Roof

Coverings

Standard for Safety for Class C Asphalt Organic-Felt Sheetroofing and Shingles

Standard for Safety for Electric Lighting Fixtures

Standard for Safety for Flexible Cord and Fixture Wire

Standard for Safety for Safety Wired Cabinets

Standard for Safety for Electric Fence Controllers

Standard for Safety for Septic Tanks, Bituminous-Coated Metal

Standard for Safety for Motor-Operated Appliances

Standard for Safety for Thermoplastic-Insulated Wires

Standard for Safety for Chimeys, Factory-Built, Residential Type and Building Heating Appliance

Standard for Safety for Tests for Flammability of Photographic Film

Standard for Safety for Portable Wood Ladders

Standard for Safety for Office Appliances and Business Equipment

Standard for Safety for Asbestos- and Asbestos-Varnished-Cloth-Insulated Wires

Standard for Safety for Torches, Oxy-Fuel Gas

Standard for Safety for Factory-Built Fireplaces

Standard for Safety for Electric Heating Pads

Standard for Safety for Varnished-Cloth Wires and Cables

Standard for Safety for Pressure Cookers

Standard for Safety for Electric Flatirons and Ironing Machines

Standard for Safety for Portable Electric Lamps

Standard for Safety for Carbon-Dioxide Fire Extinguishers

Standard for Safety for Smoke Detectors, Photoelectric Type, for Fire-Protecting

Signaling Systems

Standard for Safety for Household Electric Storage-Tank Water Heaters

Standard for Safety for Air Ducts

Standard for Safety for Portable Metal Ladders

Standard for Safety for Fuses

Standard for Safety for High-Interrupting-Capacity Class K Fuses

Standard for Safety for Household Refrigerators and Freezers

Standard for Safety for Dry-Chemical Fire Extinguishers

Standard for Safety for Liquid Fuel-Burning Heating Appliances for Mobile Homes and Travel Trailers

Standard for Safety for Gas-Heating Appliances for Mobile Homes and Travel

Trailers

Standard for Safety for Roof Jacks for Mobile Homes and Travel Trailers

Standard for Safety for Drinking-Water Coolers

Standard for Safety for Meter Sockets

Standard for Safety for Waste Disposers.

Standard for Safety for Antenna-Discharge Units

Standard for Safety for Audible Signal Appliances

Standard for Safety for Air Conditioners, Central Cooling

Standard for Safety for Grounding and Bonding Equipment

Standard for Safety for Dehumidifiers

Standars for Safety for Therapeutic Lamps

Standard for Safety for Room Air Conditioners 
Standard for Safety for Wire Connectors and Soldering Lugs

Standard for Safety for Radio and Television Receiving Appliances

Standard for Safety for Attachment Plugs and Receptacles

Standard for Safety for Electric Heating Appliances

Standard for Safety for Electric Fans

Standard for Safety for Porcelain Cleats, Knobs, and Thubes

Standard for Safety for Outlet Boxes and Fittings

Standard for Safety for Rectifiers

Standard for Safety for Fire-Detection Thermostats

Standard for Safety for Transformer-Type Arc-Welding Machines

Standard for Safety for Electric Home-Laundry Equipment

Standard for Safety for Ice Makers

Standard for Safety for Liquid-Level Gauges and Indicators for Anhydrous Ammonia and LP-Gas

Standard for Safety for Hlectric Space-Heating Equipment

Standard for Safety for Christmas-Tree and Decorative-Iighting Outfits

Standard for Safety for 2 1/2-Gallon Stored-Pressure, Water-Type Fire Extinguishers

Standard for Safety for Intrusion-Detection Units

Standard for Safety for Container Assemblies for LP-Gas

Standard for Safety for Underwater Lighting Fixtures and Junction Boxes for Swimming Pools

Standard for Safety for Electric Toys

Standard for Safety for Classification, Rating, and Fire Testing of Class A, B, and C Fire Extinguishers and for Class D Extinguishers or Agents for Use on Combustible Metals

Standard for Safety for 2 1/2-Gallon Cartridge-Operated Water-Type Fire

Extinguishers

Standard for Safety for Test Method for Fire Hazard Classification of Building Materials

Standard for Safety for Oil-Fired Boiler Assemblies

Standard for Safety for Oil-Fired Central Furnaces

Standard for Safety for Oil-Fired Floor Furnaces

Standard for Safety for Oil-Fired Recessed Heaters

Standard for Safety for Oil-Fired Unit Heaters

Standard for Safety for Oil-Fired Water Heaters

Standard for Safety for Oil-Fired Air Heaters and Direct-Fired Heaters

Standard for Safety for Fireplace Stoves

Standard for Safety for Household Dishwashers

Standard for Safety for Combination Locks

Standards for Safety for Test Methods for Fire Resistance of Roof Covering

Materials

Standard for Safety for Domestic-Type Incinerators

Standard for Safety for Cord Sets and Power Supply Cords

Standard for Safety for Household Electric Clocks

Standard for Safety for Household Electric Ranges

Standard for Safety for Beauty-Parlor equipment

Standard for Safety for Service Equipment

Standard for Safety for Flectric Dry Bath Heaters

Standard for Safety for Delayed-Action Timelocks

Standard for Safety for Oil-Burning Stoves

Standard for Safety for Vehicle Alarm Systems

Standard for Safety for Chimneys, Factory-Built, Medium Heat Appliance

Standard for Safety for Flectrically Heated Bedding

Standard for Safety for Stationary and Fixed Electric Tools

Standard for Safety for Household Burglar-Alarm System Units

Standard for Safety for Hlectric Baseboard Heating Equipment

Standard for Safety for Requirements for Non-Integral Marine Fuel Tanks

Standard for Safety for Marine Through-Hull Fittings and Sea-Valves

Standard for Safety for Marine Special Purpose Water Safety Buoyant Devices

Commercial Standard for Builders' Template Hinges

Commercial Standard for Men's Pajama Sizes - Woven Fabrics

Commercial Standard for Vitreous China Plumbing Fixtures

Commercial Standard for Wood Shingles (Red Cedar, Tidewater Red Cypress, California Redwood)

Commercial Standard for Structural Fiber Insulating Board

Commercial Standard for Hoisery Lengths and Sizes Exluding Women's

Commercial Standard for Chip Board Laminated Chip Board and Miscellaneous Boards for Bookbinding Purposes

Commercial Standard for Binders Board for Bookbinding and Other Purposes 
Commercial Standard for Enameled Cast Iron Plumbing Fixtures

Commercial Standard for Artists' Oil Paints

Comercial Standard for Porcelain-Enameled Steel Utensils

Commercial Standard for Porcelain Enameled (Glass Iined) Tanks for Domestic Hot

Water Service

Commercial Standard for Ponderosa Pine Doors

Commercial Standard for Color Materials for Art Education in Schools

Commercial Standard for Size Measurements for Men's and Boys' Shorts

Commercial Standard for Insect Wire Screening

Commercial Standard for Ponderosa Pine Windows, Sash, and Screens (Using Single Glass and Insulating Glass)

USC CS166

USC CSI71

Commercial Standard for Size Measurements for Men's Work Trousers

USC CS176

USC CS186

USC CS187

USC CS190

USC CS191

USC CS192

USC CS199

USC $\mathrm{CS}_{2} \mathrm{O}_{4}$

USC CS205

USC CS208

USC CS209

USC CS233

USC CS234

USC CS236

USC CS240

USC CS241

USC CS243

USC CS246

USC CS248

USC CS251

USC CS264

USC CS265

USC CS266

USC CS269

USC CS273

USC PSI

USC PS2

USC PS5

USC PS9

USC PSIO

USC PSII

USC PSI2

USC PSI8

USC PSI9

USC PS20

USC PS21

USC PS22

USC PS24

USC PS25

Commercial Standard for Hardwood Veneered Doors (Solid-Core, Hollow-Core, and

Panel and Sash)

Commercial Standard for Prefinished Hardboard Wall Panels

Commercial Standard for Boys' Sports-Outerwear Size Measurements

Commercial Standard for Men's Work Shirt Sizes

Commercial Standard for Wood Double-Hung Window Units

Commercial Standard for Flammability of Clothing Textiles

Commercial Standard for General Purpose Vinyl Plastic Film

Commercial Standard for Machine-Grooved Shakes and Rebutted-Rejointed Shingles

Commercial Standard for Wood Aming Window Units

Commercial Standard for Wood Casement Window Units

Commercial Standard for Standard Stock Exterior Wood Window and Door Frames

Commercial Standard for Vinyl Chloride Plastics Garden Hose

Commercial Standard for Laminated Hardwood Block Flooring

Commercial Standard for Measurements for Stretch Socks and Anklets

Commercial Standard for Mat-Formed Wood Particleboard

Commercial Standard for Aluminum Tubular Frame Screens

Commercial Standard for Aluminum Tension Window Screens

Commercial Standard for Stainless Steel Plumbing Fixtures (Designed for

Residential Use)

Commercial Standard for Steel Chain Link Galvanized Fence Fabric

Commercial Standard for Vinyl-Coated Glass Fiber Insect Screening and Louver Cloth

Commercial Standard for Hardboard

Commercial Standard for Wood Horizontal-Sliding Window Units (All Sash Operating)

Commercial Standerd for Wood Horizontal-Sliding Window Units (One or more Non-

Operating Sash)

Commercial Standard for Wood Single-Hung Window Units

Commercial Standard for Aluminum Alloy Choin Link Fencing

Commercial Standard for Expanded Vinyl Fabrics for Furniture Upholstery Use

Voluntary Product Standard for Softwood Plywood, Construction and Industrial.

Voluntary Product Standard for Standard Sizes of Blackboard Slate

Voluntary Product Standard for Porcelain Enameled Formed Steel Plumbing

Fixtures

Voluntary Product Standard for Fabrics for Book Covers

Voluntary Product Standard for Polyethylene (PE) Plastic Pipe (Schedule 40 Inside Diameter Dimensions)

Voluntary Product Standard for Polyethylene (PE) Plastic Pipe (SDR)

Voluntary Product Standard for Polyethylene (PE) Plastic Pipe (Schedules 40 and

80 - Outside Diameter Dimensions)

Voluntary Product Standard for Acrylonitrile-Butadiene-Styrene (ABS) Plastic Pipe (Schedules 40 and 80 )

Voluntary Product Standard for Acrylonitrile-Butadiene-Styrene (ABS) Plastic

Pipe (Standard Dimension Ratio)

American Softwood Lumber Standard

Voluntary Product Standard for Poly(Vinyl Chloride) (PVC) Plastic Pipe (Schedules 40,80 , and 120)

Voluntary Product Standard for Poly(Vinyl Chloride) (PVC) Plastic Pipe (Standard Dimension Ratio)

Voluntary Product Standard for Melamine Dinnerware (Alpha-Cellulose-Filled) for Household Use

Voluntary Product Standard for Heavy-Duty Alpha-Celluzose-Filled Melamine

Tableware

Voluntary Product Stendard for Mosaic-Parquet Hardwood Slat Flooring

Voluntary Product Standard for School Chalk

Voluntary Product Standard for Hinged Interior Wood Door Units 


\begin{tabular}{|c|c|c|}
\hline USC & PS36 & $\begin{array}{l}\text { Voluntary Product Standard for Body Measurements for the Sizing of Boys' } \\
\text { Apparel }\end{array}$ \\
\hline USC & PS38 & Voluntary Product Standard for Steel Bi-Fold Closet Door Units, Frames, and Trim \\
\hline USC & PS39 & $\begin{array}{l}\text { Voluntary Product Standard for Clinical Thermometers (Maximum-Self-Registering, } \\
\text { Mercury-in-Glass) }\end{array}$ \\
\hline USC & $\mathrm{PS}_{4} 4$ & Voluntary Product Standard for Paper Ice Bag Sizes \\
\hline USC & $\mathrm{PS}_{4} 5$ & $\begin{array}{l}\text { Voluntary Product Standard for Body Measurements for the Sizing of Apparel for } \\
\text { Young Men (Students) }\end{array}$ \\
\hline USC & PS49 & Voluntary Product Standard for Portable Picnic Coolers \\
\hline USC & PS51 & Voluntary Product Standard for Hardwood and Decorative Plywood \\
\hline USC & $\mathrm{R} 2$ & $\begin{array}{l}\text { Simplified Practice Recommendation for Bedding Products and Components (Mattresses, } \\
\text { Springs, Bedsteads and Cots) }\end{array}$ \\
\hline USC & R10 & Simplified Practice Recommendation for Milk and Cream Bottles \\
\hline USC & $\mathrm{R} 46$ & Simplified Practice Recommendation for Tissue Wrapping Paper \\
\hline USC & R78 & $\begin{array}{l}\text { Simplified Practice Recommendation for Iron and Steel Roofing, Siding, and Ridge } \\
\text { Roll }\end{array}$ \\
\hline USC & R114 & Simplified Practice Recommendation for Gummed Kraft Paper Sealing Tape \\
\hline ISC & $\mathrm{R} \geq 74$ & Simplified Practice Recommendation for Cast-Iron Radiators \\
\hline USC & Rl75 & $\begin{array}{l}\text { Simplified Practice Recommendation for Heavy-Duty, Round, Nesting, Paper, Food and } \\
\text { Beverage Containers and Lids }\end{array}$ \\
\hline USC & R179 & $\begin{array}{l}\text { Simplified Practice Recommendation for Structural Insulating Board (Wood or Cane } \\
\text { Fiber) }\end{array}$ \\
\hline USC & R187 & $\begin{array}{l}\text { Simplified Practice Recommendation for Food Trays, or Dishes (Waxed Paper, Molded } \\
\text { Wood Pulp, and Wood Types) }\end{array}$ \\
\hline USC & R192 & $\begin{array}{l}\text { Simplified Practice Recommendation for Crayons and Related Art Materials for } \\
\text { School Use (Types, Sizes, Packaging, and Colors) }\end{array}$ \\
\hline USC & R257 & $\begin{array}{l}\text { Simplified Practice Recommendation for Thermal Conductance Factors for Preformed } \\
\text { Above-Deck Roof Insulation }\end{array}$ \\
\hline VCMA & E1 & $\begin{array}{l}\text { Vacuum Cleaner Standard; Method of Measuring Motor Horsepower Output of Canister } \\
\text { and Tank Type Household Vacuum Cleaners }\end{array}$ \\
\hline $\begin{array}{l}\text { WCF } \\
\text { WSC }\end{array}$ & S100 & $\begin{array}{l}\text { Industry Standard for Household, Commercial and Portable Exchange Water Softeners } \\
\text { Testing and Rating Standards of the Water Systems Council }\end{array}$ \\
\hline WSFI & $* 4$ & $\begin{array}{l}\text { Specifications for Rubber Cushion-Sleeper Construction With Nailers and Finish } \\
\text { Flooring }\end{array}$ \\
\hline WSFI & *5 & Specifications for Steel-Splined Continuous Strip Mastic-Set Maple Flooring \\
\hline WSFI & *6 & $\begin{array}{l}\text { Specifications for Mastic-Nailed Construction With Mastic-Set Subflooring and } \\
\text { Nailed Finish Flooring }\end{array}$ \\
\hline WSFI & *7 & Specifications for Mosaic Wood Parquet Flooring Set in Adhesive \\
\hline WSFI & *8 & $\begin{array}{l}\text { Specifications for Mastic Cushioned Construction with Nailers, Subflooring and } \\
\text { Finish Flooring }\end{array}$ \\
\hline WWPA & *23 & Standard Grading Rules for Western Lumber \\
\hline
\end{tabular}


The applicable Standard Industrial Classification (SIC) number(s) immediately follow(s) the product entry in parentheses. There are some instances when a SIC number courd not be assigned. The assignment of the SIC number(s) was based only on the first product listed in the entry and all words describing that product; for example:

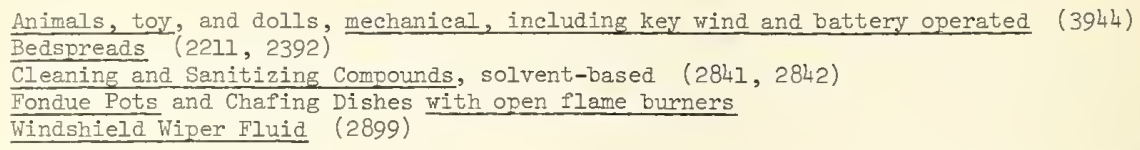

When more than one SIC number is provided, each number should be checked and identified before being used for statistical purposes since the SIC number(s) can refer to (1) different materials, (2) products made from purchased materials transferred from another establishment, (3) products made in the same establishment as the basic materials, or (4) separate parts of products. In the latter case, the numbers are separated by "and;" for example: Garage doors, with automatic door opener and closer (2431, 3442, and 3699) (2431, 3442 refer to the garage door and 3699 refers to the automatic door opener and closer $\longdiv { \text { . } }$ 
Automotive Chemicals, including brakefluid, oil, aditives (2899, 2992)

B-B Guns (3484) . . . . . . . . . . . . . . . . . . 1237

Baby Baths . . . . . . . . . . . . . . . . . . . . . 1514

Baby Carriers - bicycle accessory . . . . . . . . . . . . . . . . . . . . . . . 1531

Baby Harnesses . . . . . . . . . . . . . . . . . . . . . . . 1524

Baby Items - see category 1500 or under (baby) item

Backyard Equipment - see Playground Equipment not elsewhere classified 1201

Badminton Equipment (3949) . . . . . . . . . . . . . . . . . 1219

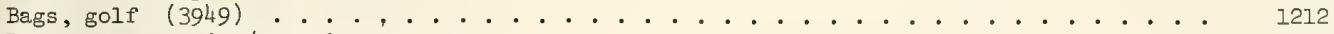

Bags, oven cooking/roasting . . . . . . . . . . . . . . . . . . . 1113

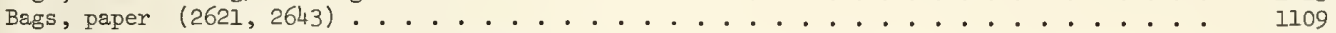

Bags, plastic, trash, clothes, and garden . . . . . . . . . . . . . . 1108

Bags/Luggage, Suit Bag $(3161)$. . . . . . . . . . . . . . . . . . 1109

Balconies $(3446) \ldots \ldots \ldots \ldots \ldots \ldots 1 . \ldots \ldots 17$

Balloons $(3069,3079)$. . . . . . . . . . . . . . . . . . . . . 1347

Balls and Paddles, ping pong (3949) . . . . . . . . . . . . . . . . . . . 1269

Balls, Cues, Chalk for Billiards (3949) . . . . . . . . . . . . . . . . . . . 1260

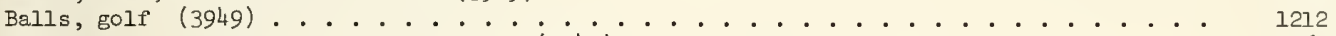

Balls, sports, not otherwise specified (3949) . . . . . . . . . . . . 1265

Balls, tennis (3949) . . . . . . . . . . . . . . . . . . . . . . 1219

Balls, volleyball $(3949)$. . . . . . . . . . . . . . . . . . . . . 1266

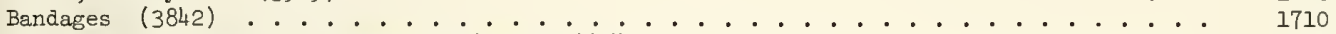

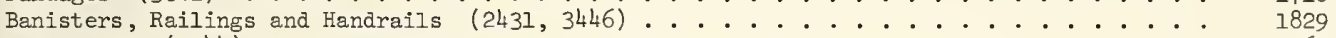

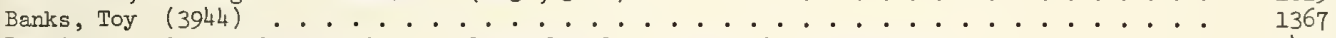

Bar Accessories; swizzle sticks, seltzer bottles, manual ice crackers, etc. . . . . . 0439

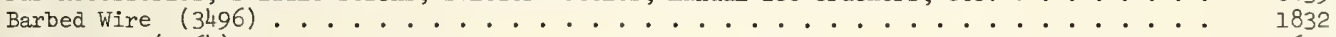

Barrettes $(3964) \ldots \ldots$

Bars and Bar Stools $(2511,2514,2519) \ldots \ldots 0658$

Baseball Equipment, including baseballs, bats, "bases," masks, and other
protective gear; apparel (3949) . . . . . . . . . . . . . . . 1204

Basketball Equipment (3949) . . . . . . . . . . . . . . . . . . . 1205

Baskets, bicycle $(3496)$. . . . . . . . . . . . . . . . . . . . . . 1202

Baskets, Easter $(2499,3944) \ldots \ldots \ldots \ldots . \ldots \ldots 1714$

Baskets, laundry $(2499)$. . . . . . . . . . . . . . . . . . . . 0438

Baskets, picnic (2499) . . . . . . . . . . . . . . . . . . 1228

Baskets, trash $(2655)$. . . . . . . . . . . . . . . . . . . . . . . . . . . . . . . .

Bassinettes . . . . . . . . . . . . . . . . . . . . . 1529

Bathinettes . . . . . . . . . . . . . . . . . . 1530

Baths, baby . . . . . . . . . . . . . . . . . . . . . . 1514

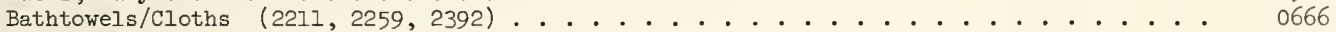

Bathtub and Shower Enclosures, glass (3231) . . . . . . . . . . . . 0609

Bathtub and Shower Enclosures of Materials Other Than Glass . . . . . . . . . . . . . 0610

Bathtub and Shower Structures Other Than Doors and Panels, including the

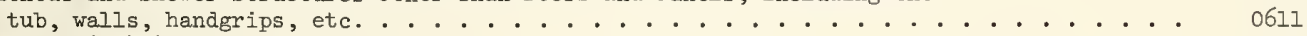

Batons $(3949)$. . . . . . . . . . . . . . . . . . . . 1271

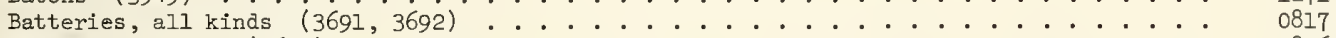

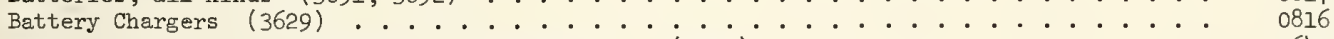

Beach Chairs, Folding Furniture, Patio Furniture $(2517)$. . . . . . . . . . . . 0643

Beach Equipment . . . . . . . . . . . . . . . . . . . . . . . 1222

Beach Towels $(2211,2259,2392)$. . . . . . . . . . . . . . . . . . 0666

Beads $(3911,3961) \ldots \ldots \ldots 1616$

Bedding, baby . . . . . . . . . . . . . . . . . . . . . 1507

Bedding, including mattresses, mattress covers and pads . . . . . . . . . . . . 0642

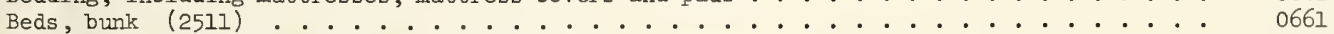

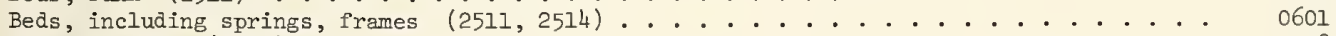

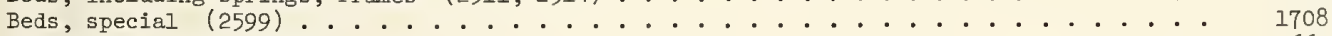

Bedsprings $(2211,2392) \ldots \ldots . \ldots \ldots 667$

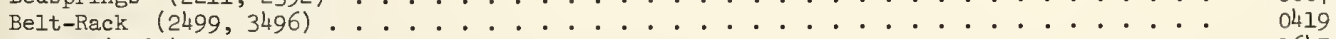

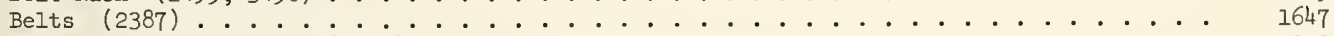

Bicycles and Equipment $(3751)$. . . . . . . . . . . . . . . . 1202

Bikes, exercise $(3949)$. . . . . . . . . . . . . . . . . 1209

Bikes, Mini-bikes $(3751)$. . . . . . . . . . . . . . . . . 1263

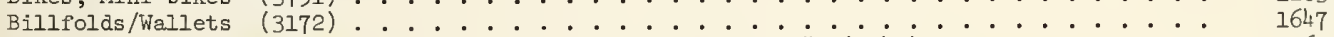

Billiards, including tables, balls, cues, chalk, "pool table" $(3949) \ldots \ldots$ 
Blankets, electric (3634)

Blinds, window, venetian (2591)

Blocks, Pull Toys, and Similar Items $(3069,3944)$

Boards, exercise equipment (3949)

Boards, Lumber, Paneling Pieces, not part of structure $(2421,2426,2491) \ldots 1838$

Boats, Motors and Accessories for Recreational Use (3731) . . . . . . . . . . . . 1203

Boats, not including models (3944)

1203

Bobbins for Sewing Machines (3636)

0112

Boilers (3433)

Book Shelves, freestanding (2541)

Books (2731)

Books, children's $(2731,3944)$

0308

0664

0668

Boots, ski (3i ${ }^{\circ}$

Bottle Cutting Equipment/Kits

(3423)

1366

1615

1216

0532

Bottle Openers $(3469,3496)$

Bottle Warmers (3079)

Bottles, aerosol

Bottles, glass, except soft drink bottles (3221)

0422

1510

Bottles, glass, soft drink (2086, 3221)

1101

Bottles, Nipples and Related Items

1106

Bottles, seltzer (3221)

Bows and Arrows

Bows and Arrows, toy (3944)

1110

Bowling Equipment (3949)

Breakers, circuit.

\section{Bridles (3199)}

Brief Cases (3161)

Briquettes/Charcoal (2819)

Broiler Pans, oven accessories (363i, 3634)

1811 
Cabinet Pulls (3429)

Cabinets, ammunition, gun (2541)

Cabinets, medicine (2514)

Cabinets, Shelves and Storage Areas (2434, 2514, 2517, 2519)

Cages, pet (3496)

Cake Decorators (3469)

Cameras, movie ( 3861 )

1715

0428

Cameras, still picture and slide (386i)

Camping Clothing (2329, 2339) . . . .

Camping Equipment, including tents, cots and sleeping bags

Camping Trailers (3792)

Can Openers, powered (3634)

Can Openers, unpowered (3423) ... . .

0401

Candles, butane

Candles, wax (3999)

Candlesticks (3229, 3914)

Canes, Crutches and Walkers

Canisters and Similar Containers (3079, 3229)

Canners

Cannons, cap, toy (3944)

0433

0423

0415

0433

1706

0435

0412

1348

Cans, metal (3411)

Caps and Cap Toys (guns, rifles, rockets, cannons) (2899)

1112

Caps/Hats (2352)

1348

Car Seats, baby

Carbon Tetrachloride (2869)

1647

1519

Cardboard Boxes (2631, 2651)

0928

1114

Carpet Tacks (3315, 3399)

Carpeting, including outdoor carpeting but excluding runners (227i)

1819

Carriages, baby (3949)

0613

1505

Carriages, doll (3944)

1340

Carriers, baby - bicycle accessory

Carriers, baby (not back packs or slings)

1531

1503

"Carriers," infant, personal use (back packs, slings, etc.). . . . . . . . . . . . . . 1527

Carriers, luggage, personal use . . . . . . . . . . . . . . . . . 1631

Carriers, pet... . . . . . . . . . . . . . . . . . . . . . . . 1715

Cars and Trucks, toys, not including models (3944) . . . . . . . . . . . . . . . . 1305

Cars, models, gasoline powered (3944) . . . . . . . . . . . . . . . . . . . 1306

Carts, grocery, personal use (3496) . . . . . . . . . . . . . . . . . . . 1631

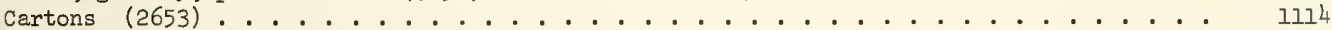

Caustics, lye (2842)

Caulking/Spackling Compounds isio

Ceramic, Pottery and Clay Supplies and Equipment (not including tableware) (3952)

0916

0920

Cesspools, Cisterns, and Septic Tanks

0514

Chafing Dishes and Fondue Pots with Open Flame Burners

Chain Locks (3429)

Chain Saws (3546)

Chains, heavy duty ( $3 \dot{4} 6 \dot{2})$

Chains, tire (3496)

Chairs, beach (2519)

Chairs, highchairs

Chairs, not upholstered or not otherwise specified (2512)

Chairs, recliner (2512)

Chairs, rocking (2511)

Chairs, upholstered (25i2)

Chairs, youth (2511)

Chalk, Cues, Balls for Billiards (3949)

Chandeliers (3645)

Changing Tables, baby .

Charcos./Briquettes (2819)

Charcoal. Grills, stationary/"built-i"

Charcoal Iighter Fuel (2899)

Chargers, battery (3629)

Chemicals, automotive (2899, 2992)

0402

0707

1411

0814

0821

0643

1501

0644

0670

0671

0602

1518

1260

0628

1502

0917

1249

1247

0816

0939

0921

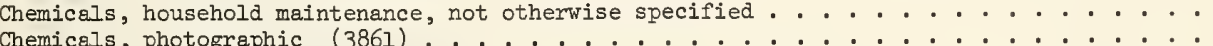

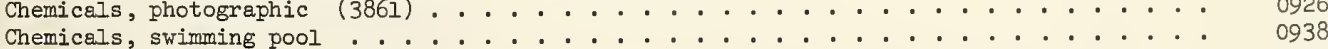

Chemistry Sets, toy $(3944)$. . . . . . . . . . . . . . . . . . . . . 1315

Chests $(2511)$. . . . . . . . . . . . . . . . . . . . . . . . . . . . . . . . . . . 
Chests or Boxes, toy (2511, 3944)

Children's Books (2731, 3944)

Chimneys, factory built

Chisels (3423)

Choppers/Slicers, electric (3634)

Chopping Devices (3469)

Christmas Tree Lights (3699)

Christmas Tree Ornaments and Other Decorations (3229, 3231, 3999)

Christmas Tree Stands and Supports (2499)

Christmas Trees, artificial (3962)

Circuit Interrupters and Breakers

Cisterns, Cesspools and Septic Tanks

Clacker Balls (3944)

Clay, Pottery and Ceramic Supplies and Equipment (not including tableware) (3952)

Cleaners, oven (2842)

Cleaners/Shampooers, rug, electric (3639)

Cleaners, vacuum (3635)

Cleaners, wallpaper (2842)

Cleaning Agents and Compounds (soap, cleanser, laundry detergent) (284i)

Cleaning and Sanitizing Compounds, solvent-based (2841, 2842)

Cleaning Equipment, including brooms, mops, brushes, cleaning pads, buckets, wash tubs, etc.

Cleaning Fluids (2842)

Cleansers (2841)

Climbing Apparatus, "Monkey Bars", "gyms" (3949)

Clips, hairclips (3964)

Clocks, electric (3873)

Clocks, other than electric (3873)

Closers and Openers, door, automatic (3699)

Closures, child resistant

0117

Closures, resealable (3079)

Clothes and Accessories for Dolls (3942)

1105

1104

Clothes Dryers - see Dryers

Clothes Hangers, all types (2499, 3079, 3496)

1364

Clothes Lines and Collapsible Dryers

Clothes steamers, personal use (3634)

0419

1821

0255

Clothes Washers - see Washing Machines

Clothesbrushes (3999)

Clothespins and Similar Fasteners (2499, 3079)

Clothing Accessories, including gloves/mittens, scarves, hats/caps, handbags, belts,

wallets/billfolds, etc.

Clothing, baseball apparel and caps (2329; 2352)

Clothing, day wear (underwear, skirts, slacks, blouses, shirts, dresses, socks/hose, suits, sweaters, vests)

Clothing, football apparel (2329)

Clothing for Camping or Sports, specialized (2329, 2339 )

Clothing for Mountain Climbing (2329, 2339)

Clothing, hockey (2329)

Clothing, horseback riding outfits (2329, 2339)

Clothing, outerwear (coats, raincoats, jackets, snow suits, ponchos, parkas) (2311, 2337 ) . 1646

Clothing, scuba diving

Cloths, bath and disheloths (2211, 2259, 2392)

Clubs, golf (3949)

Coats $(2311,2329,2337,2363,2371,2386)$

Cocktail Tables (2511, 2519, 3231)

Coffee Grinders, non-electric

Coffee Tables (2511, 2519, 3231)

Coffeemakers, electric (3634)

Coffeemakers, unpowered (3469)

Collapsible Dryers and Clothes Lines (2499)

Collars, pet (3199)

Combs, electric (3634)

Combs/Hair Brushes, not powered (3999)

Comforters (2392)

Compactors, garbage/trash (3639)

Compounds, cleaning and sanitizing, solvent-based (284l, 2842) 
Construction Materials, other, weather stripping, sheet metal . . . . . . . . . . . . 1833

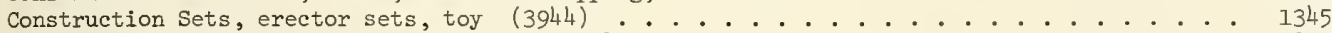

Construction Wire . . . . . . . . . . . . . . . . . . . . . . . 1832

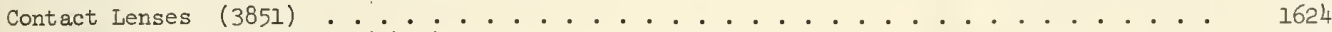

Containers, aluminum foil (3497) . . . . . . . . . . . . . . . . 1115

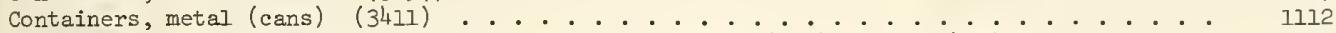

Containers, other, except vacuum or pressure containers $(2654,2655,3412) \ldots . . . . .1107$

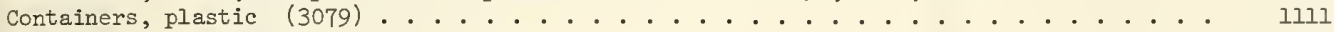

Containers, pressurized, aerosol cans . . . . . . . . . . . . . . . . 1101

Containers, vacuum $(3429)$. . . . . . . . . . . . . . . . 1102

Containers, waste, trash can/basket, etc. $(3469)$. . . . . . . . . . . . 0413

Convertible Sofa Beds (2515) . . . . . . . . . . . . . . . . 0647

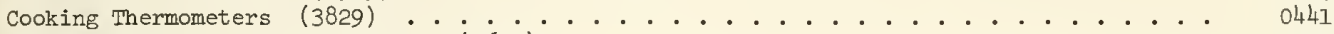

Cooking Devices, battery powered (3631) . . . . . . . . . . . . . . . 1227

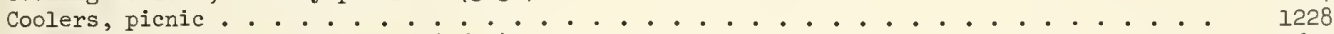

Cords, appliance and extension (3699) . . . . . . . . . . . . . . . 0621

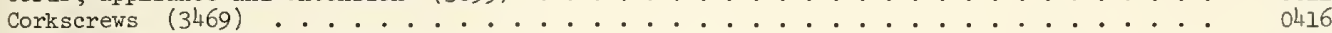

Corn Poppers, electric $(3634)$. . . . . . . . . . . . . . . . . . . . . . . . . . . . . .

Corrugated Boxes/Cartons (2653) . . . . . . . . . . . . . . . 1114

Cosmetic Bags, luggage $(3161)$. . . . . . . . . . . . . . . . . . . 1623

Costumes (Halloween, etc.), including masks, wigs . . . . . . . . . . . . 1342

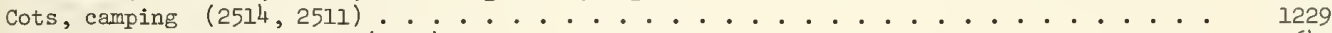

Couches, Sofas, Sofa Beds (2515) . . . . . . . . . . . . . . . . 0647

Covers and Pads, mattress (2392) . . . . . . . . . . . . . . . . 0642

Covers for Ironing Boards $(2392)$. . . . . . . . . . . . . . . . . . . . 0408

Covers, patio and porch $(2394,3079)$. . . . . . . . . . . . . . . 1809

Covers, slip covers (2392) . . . . . . . . . . . . . . . . . . . . . . . . . . . . . . . . . . . .

Crayons and Children's Paint sets (3952) . . . . . . . . . . . . . . . 1351

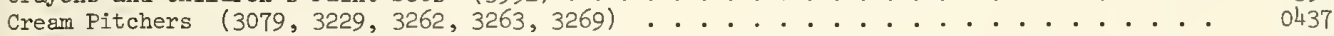

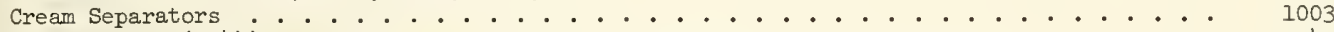

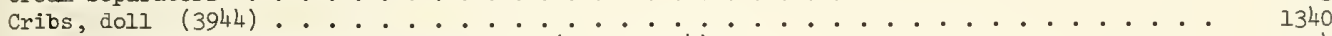

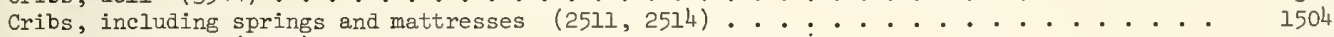

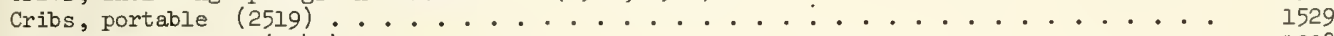

Croquet Equipment $(3949)$. . . . . . . . . . . . . . . . . . . . . . 1208

Crutches, Canes and Walkers $(3842)$. . . . . . . . . . . . . . . . 1706

Cues, Balls, Chalk for Billiards (3949) . . . . . . . . . . . . . . . 1260

Cultivators and tillers, power (not farm equipment) (3524) . . . . . . . . . . 1408

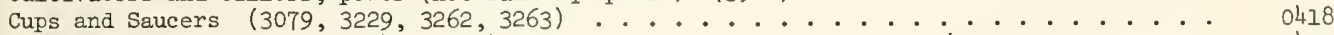

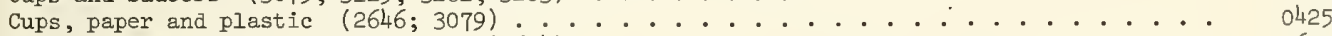

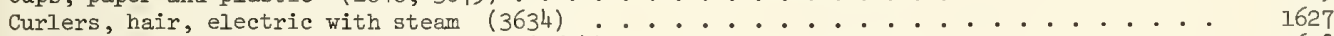

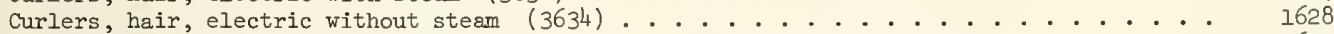

Curlers, hair, non-electric (3964) . . . . . . . . . . . . . 1603

Curtain and Drapery Rods, all kinds (2591) . . . . . . . . . . . . . 0655

Curtains, Drapes, including plastic curtains (2391) . . . . . . . . . . . 0617

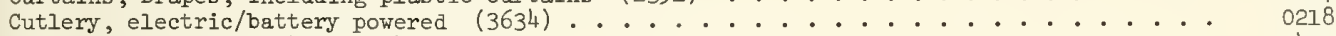

Cutlery, unpowered $(3421,3914)$. . . . . . . . . . . . . . . . 0407

Cutters, Wire (3423) . . . . . . . . . . . . . . . 0810

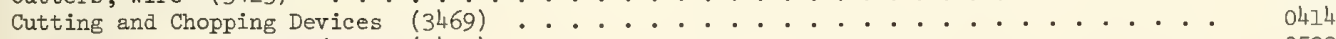

Cutting Glass Equipment/Kits $(3423)$. . . . . . . . . . . . . 0532

Darts and Dart Games, lawn darts, targets (3944) . . . . . . . . . . . . 1248

Day Wear (underwear, skirts, slacks, blouses, shirts, dresses, socks/hose, suits, sweaters, 1645

Decorations, Christmas tree (3999) . . . . . . . . . . . . . . . 1713

Decorations, other seasonal, Halloween, Easter baskets, etc. . . . . . . . . 1714

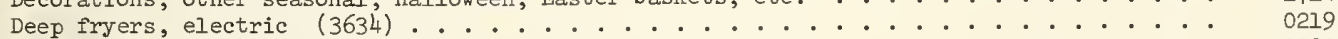

Defroster Devices, electric $(3634)$. . . . . . . . . . . . . . . . 0220

Dehumidifiers $(3634)$. . . . . . . . . . . . . . . . . . . . . . . . . . . . . . . . . .

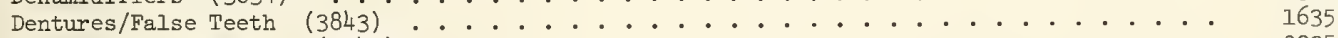

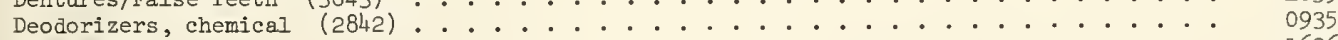

Desk Supplies . . . . . . . . . . . . . . . . . . . 1626

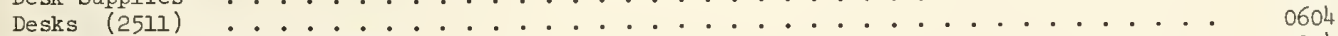

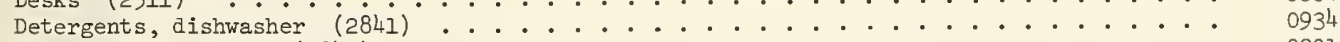

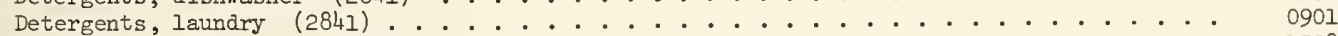

Diaper Pails . . . . . . . . . . . . . . . . . . . . . . . . 1528

Diaper Pins and Fasteners $(3964)$. . . . . . . . . . . . . . . . 1523

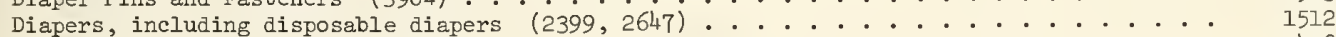

Dishes $(3079,3229,3262,3263) \ldots \ldots \ldots . \ldots \ldots \ldots . \ldots \ldots$

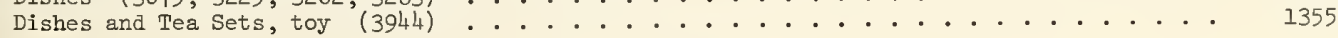


Diving Boards : '

Dog Houses (2452, 3448 )

Doll Clothes and Accessories (3942)

Doll Houses and Doll Furniture (3944)

Dolls and Toy Animals, mechanical including keywind and battery operated (3942)

Dolls and Toy Animals, non-mechanical (3942)

Door Mats (2279)

Door Springs (3495)

Door Stops

Doorknobs (3429)

Doors, Garage, with automatic door opener and closer (2431, $344 \dot{2}^{\circ}$ and $\left.369^{\circ}\right)$

Doors, garage (2431, 3442)

Doors, glass, not otherwise specified (3231)

Doors, jalousie, glass (2431)

0117

Doors, screen (2431, 3442)

1822

1837

Doors, storm, glass (2431)

Doors (swinging, folding, track, panel, trap, hatch, etc.) (2431, 3442 )

Ductwork for Heating or Cooling Systems (3444)

Drain Cleaners (2842)

Drain Pipes/Spouts

Drapes, Curtains, including plastic curtains (2211, 2221, 2391)

Drapery Hooks/Rings, Shower Curtain Hooks (2591)

Dressers (2511)

Dresses $(2335,2361)$

Dressing Gowns (2384)

Drill Attachments, power (3545)

Drills, power (3546)

Drinking Glasses, glass (3229)

Drinking Cups and Glasses, non-glass $\left(2646,307 \dot{9}, 326 \dot{9}^{\circ}\right.$

Dryers, collapsible and clothes lines (2499)

Dryers, electric, without washing machines attached (3633)

Dryers, gas, without washing machines attached (3633).

Dryers, hair (3634)

Dyes and Bleaches, not intended for cosmetic use (2899)

Ear Protection Devices, including noise plugs (3842)

Electrical Distribution Systems 
Exercisers, baby . . . . . . . . . . . . . . . . . . 1520

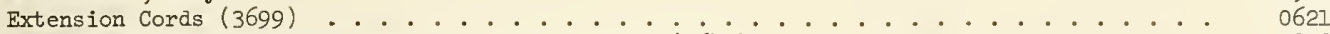

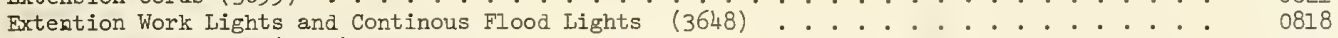

Extinguishers, fire (3999) . . . . . . . . . . . . . . . . . . 0701

Eye Protection Devices, light shields, sunglasses (3851) . . . . . . . . . . 1607

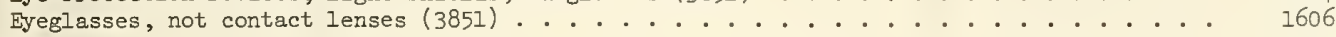

Fabrics, not otherwise specified . . . . . . . . . . . . . . 0635

Falls, Hairpieces $(3999)$. . . . . . . . . . . . . . . . . . . . . 1605

False Teeth/Dentures (3843) . . . . . . . . . . . . . . . . . . . . . . . 1635

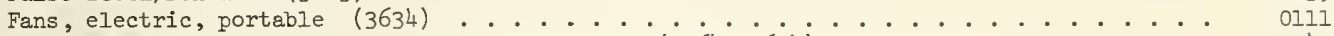

Fans, permanently installed including attic fans $(3564,3634) \ldots \ldots 341$

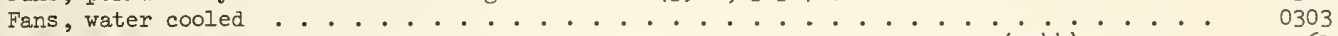

Farm Yards, Spaceports, Service Stations, Parking Garages and Similar Toys (3944) . . . . 1361

Fasteners and Diaper Pins (3964) . . . . . . . . . . . . . . . . . 1523

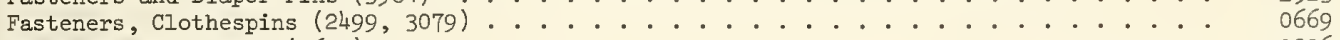

Faucet Water Heaters $(3639)$. . . . . . . . . . . . . . . . . . . . 0236

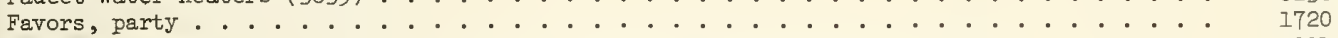

Fences, electric . . . . . . . . . . . . . . . . . . 1001

Fences, not electric, outdoor, all types including posts $(2499,3446,3496) \ldots . . .2834$

Fertilizer, plant food (2873). . . . . . . . . . . . . 1429

Files, workshop (3423) . . . . . . . . . . . . . . . . . . . 0810

Film for All Cameras $(3861)$. . . . . . . . . . . . . . . . . 0534

Filter Pumps, Frames, and Other Equipment for Swiming Pools - above ground . . . . . . 1262

Filters for Aquariums . . . . . . . . . . . . . . . . . . . . 1240

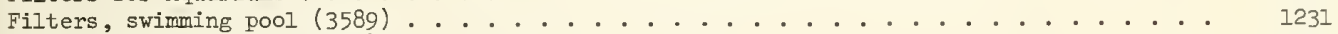

Fire and Smoke Alarms $(3829)$. . . . . . . . . . . . . . . . . . . . 0702

Fire Escape Devices, including chain ladders $(3446) \ldots \ldots 0703$

Fire Extinguishers (3999) . . . . . . . . . . . . . . . . . . . 0701

Fireplace Equipment, including andirons, screens, tools, grates, non-wood iogs (3429). . 0663

Fireplaces, factory built, gas burning . . . . . . . . . . . . . 0334

Fireplaces, factory built, wood burning . . . . . . . . . . . . . . 0316

Fireplaces, individually built . . . . . . . . . . . . . . . . 1802

Fireworks $(2899)$. . . . . . . . . . . . . . . . . . . . . . . . . 1313

First-Aid and Health Equipment, including hot water bottles, thexmometers, Q-tips,

bandages (not medications, home use) (3842) ....................... 1710

Fish Ponds $(3272) \ldots \ldots \ldots \ldots . \ldots \ldots . . \ldots \ldots 147$

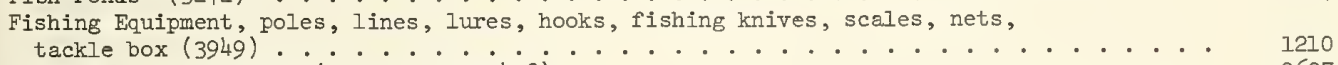

Fittings for Gas Pipes $(3079,3312,3498) \div 0607$

Fixtures, light $(3645)$. . . . . . . . . . . . . . . . . . . . . . . . . . . . . . . . . . . . . . .

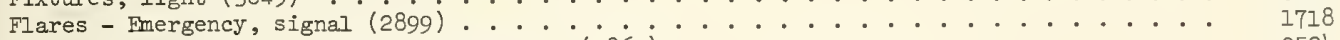

Flash Attachments for Cameras, Flash Bulbs (3861) . . . . . . . . . . . . 0524

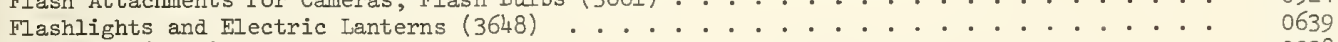

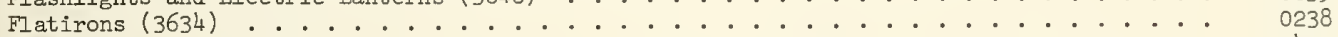

Flats, greenhouse $(2441)$. . . . . . . . . . . . . . . . . 1413

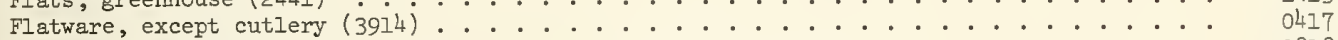

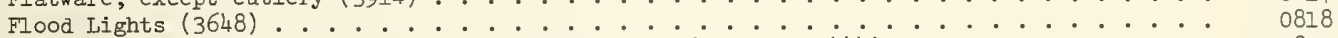

Floors and Flooring Materials $(2421,2426,2491,3069,3251,3444) \ldots \ldots . . . . . .2807$

Flower Pots $(3079,3269)$. . . . . . . . . . . . . . . . . 1413

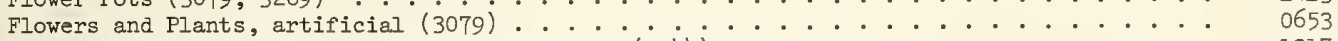

Flying Devices, not gasoline or rocket powered (3944) . . . . . . . . . . . 1317

Foil, Aluminum Wrapping products (3497) . . . . . . . . . . . . . . . 1115

Fondue Pots and Chafing Dishes with open flane burners . . . . . . . . . . . . 0402

Food Grinders, manual . . . . . . . . . . . . . . . . . . 0411

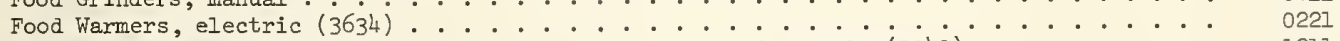

Football Equipment, apparel, footware, protective gear, footbalis (3949) . . . . . 1211

Footstools, Ottomans, Hassocks (2512) . . . . . . . . . . . . . 0665

Footwear/Boots, ski (3149) . . . . . . . . . . . . . . . . . 1216

Footwear/Cleats/Shoes, footbali $(3149)$. . . . . . . . . . . . . 1211

Footwear (except for sports footwear) . . . . . . . . . . . . . . 1615

Footwear/Shoes, baseball $(3149)$. . . . . . . . . . . . . . . . . 1204

Footwear/Shoes, golf $(3149)$. . . . . . . . . . . . . . . . . 1212

Forks, spoons $(3914)$. . . . . . . . . . . . . . . . . . . . 0417

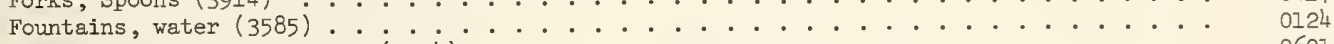

Frames and Springs for Beds $(2514)$. . . . . . . . . . . . . . . . 0601

Frames, filter pumps for above ground swimming pools . . . . . . . . . . . . . 1262 
Freezers, gas, separate (3632)

Freezers, not otherwise specified (3632)

Fry Pans, electric (3634).

Fryers, deep, electric (3634)

Fuels for Chafing Dishes, Fondue Pots, and Similar Items

Fuels for Model Cars, Airplanes, Etc.

Fumigants (2842)

Furnaces, coal (3433)

Furnaces, electric

Furnaces, floor, cosi (3433)

Furnaces, floor, electric.

Furnaces, floor, gas (3433)

Furnaces, floor, not otherwi

Furnaces, floor, oil (3433)

Furnaces, gas (3433)

Furnaces, not otherwise specified

Furnaces, oil (3433)

Furniture - beach chairs, folding, patio (2511, 2519)

Furniture, inflatable

Furniture, not otherwise specified

Furniture, nursery and equipment - other

Gadgets, kitchen, including mixing and measuring spoons/cups, strainers, spatulas,

rolling pins, cake decorators, etc. (3469)

0428

Games, adult and novelty items (3944)

Games, dart, lawn darts, targets (3944)

Games, electric (3944)

Games and Game Parts, other than electric (3944)

Garage Doors (2431, 3442)

Garage Doors with Automatic Door Opener and Closer (2431, 3442 and 3699)

Garages, Tool Sheds, Dog Houses and Other Pet Enclosures, separate (3448)

Garbage Disposers ( 3639 )

Garbage/Trash Compactors (3639)

Garden and Greenhouse Supplies

Garden Hoses, Nozzles and Sprinklers (3041)

Garden Tools (3423)

Gas Grills, stationary/"built-in"

Gas Lamps, installed (3648)

Gas (IP) Heating Equipment (3433)

Gas Pipes, Fittings and Distribution Systems (3079, 3312, 3498 )

Gas Tanks and Fittings, propane (IP) (3443)

Gasoline (2911)

Gasoline Powered Toys, including model airplanes and cars $(3944)$

Gates, baby

Generators/Electric Power Plants

Glass Bathtub and Shower Enclosures (3231)

Glass Bottles and Jars, except soft drink bottles (3221)

1721

1248

1307

1320

1822

0117

1830

0237

0252

1413

1414

1403

1250

0625

1256

0607

0131

0910

1306

1506

0606

0609

1106

Glass Bottle Cutting Equipment/Kits (3423)

Glass Doors, not otherwise specified (3231)

0532

Glass, mirror (3231)

Glass Panels, fixed (323i)

1837

0640

Glass Panels, sliding (3231)

1824

Glass Soft Drink Bottles ( 2086

1825

1110

Glass Storm Doors (2431)

1823

Glass Tables - all kinds (3231)

Glass, window (3211)

1815

Glasses, drinking glass (3229)

Glasses, drinking, non-glass (264., 3079, 3269)

Glasses/Eyeglasses (3851) 
Golf Carts (3949)

Golf Equipment except carts, including balls, clubs, bags, shoes (3949)

Grass/Leaf Catcher Attachments for Mowers (3524)

Graters/Slicers, vegetable (3469)

Grates for Fireplaces (3429)

Greenhouse and Garden Supplies

Griddles, electric (3634)

Grills and Broilers, electric, counter-top ovens (3634)

Grills, charcoal, stationary/"built-in" (3631)

Grills, gas, stationary/"built-in"

Grills, outdoor, not otherwise specified (3631)

Grills, portable, charcoal, "Hibachi" (3631)

Grills, portable, gas (3631)

Grills, portable, kerosene (3631)

Grills, stationary, outdoor, kerosene (363i)

Grills/stoves, portable, gasoline, for cooking (3631)

Grinders, coffee, non-electric

Grinders, food, manual

Grinders/Leaf Mulchers, powered, "Shreader-Bagger" (3524)

Grinders, meat, electric (3634)

Grinders, power (3541)

Grocery Carts and Luggage Carriers, personal use (3496)

Ground Fault Circuit Interrupters

Grounding Devices (3643)

Gun Cabinets (2541, 2542)

Guns, cap toy (3944)

Guns, gas, air and spring operated including $\mathrm{B}-\mathrm{B}$ guns (3484)

Guns, soldering (2423)

Guns, toy and Other Toy Weapons with Projectiles (3944)

Guns, toy and Other Toy Weapons without Projectiles (3944)

Gutters, Drain Pipes/Spouts, Run-0ff Pipes

Gymnastics and Associated Equipment, including mats, rings, parallel bars, balance beams (3949)

"Gyms" (3949)

Gyms, Crib

Hair Accessories, bands, barrettes, decorative clips, etc. (3999)

Hair Brushes/Combs, not powered (3999).

Hair Curlers, electric with steam (3634).

Hair Curlers, electric, without steam (3634)

Hairpins (3964)

Halloween Decorations (2646)

Hammers (3423)

Hammocks $(2399,2514)$

1628

Hampers, Laundry (2499)

Health and First-Aid Equipnent, including hot water bottles, thermometers, Q-tips,

bandages (not medications, home use) (3842) . . . . . . . . . . . 1710

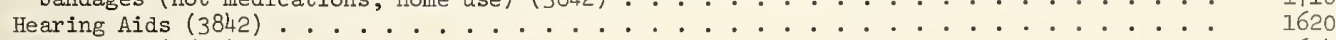

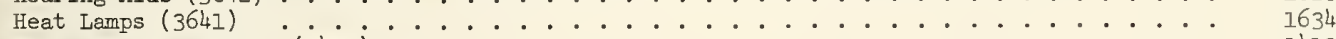

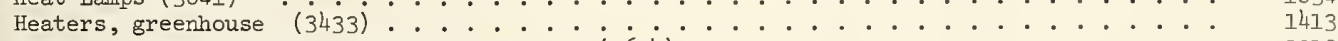

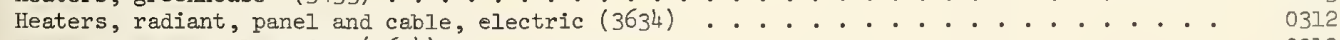

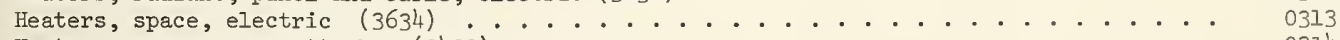

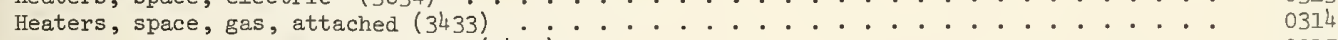

Heaters, space, kerosene, attached $(3433)$. . . . . . . . . . . 0315

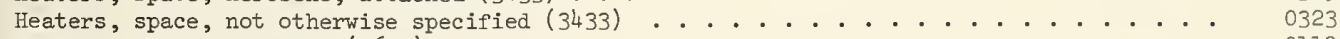

Heaters, water, electric $(3639)$. . . . . . . . . . . . . . 0119

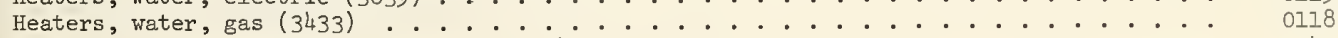

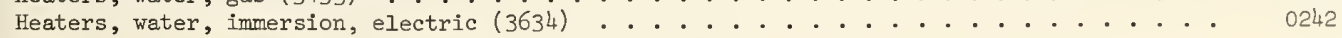


Heaters, water, not otherwise specified (3639) . . . . . . . . . . . . . . . . 0128 Heaters, water, oil (3633) . . . . . . . . . . . . . . . . . . 0120

Heating Equipment, portable, alcohol (3433) . . . . . . . . . . . . . . . .

Heating Equipment, portable, gas (IP) (3433) . . . . . . . . . . . . . . . . . . 1256

Heating Equipment, portable, gasoline (3433) . . . . . . . . . . . . . . . . . . . 1225

Heating pads, electric $(3634)$. . . . . . . . . . . . . . . . . 0110

Heating Stoves, gas (3433) . . . . . . . . . . . . . . . . . . . . . 0330

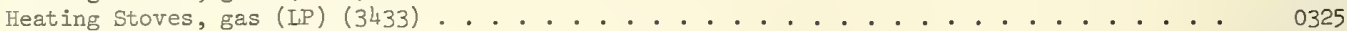

Heating Stoves, kerosene (3433) . . . . . . . . . . . . . . . . . . 0331

Heating Systems, other, including heat pumps (3433) . . . . . . . . . . . . . . . . 0 . . . . .

Hedge Trimmers, powered (3524) . . . . . . . . . . . . . . . . . . . . . 1427

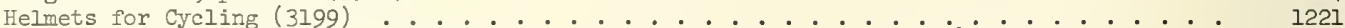

Hi Fi and Stereo Components and Equipment, including amplifiers, remote speakers (3651) . . 0520

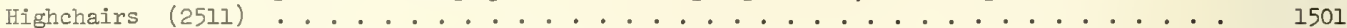

Hinges (3429) .. . . . . . . . . . . . . . . . . . . . . . . 1820

Hobby Horses and Similar Child Carrying Toys (3944) . . . . . . . . . . . . . . . . . . 1327

Hockey Equipment, includes apparel and protective gear (3949) . . . . . . . . . . . . 1214

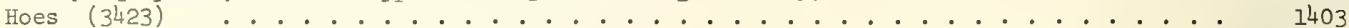

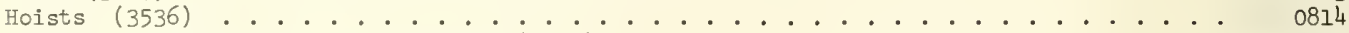

Hooks for Drapery and Shower Curtains (2591) . . . . . . . . . . . . . . 0673

Horns, bicycle (3751) . . . . . . . . . . . . . . . . . . . . . . 1202

Hors d'Oeuvre Picks $(2499,3079)$. . . . . . . . . . . . . . . . . . . . . 0431

Horseback Riding Equipment and Accessories, including apparel (3949) . . . . . . . . . 1239

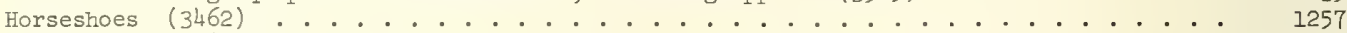

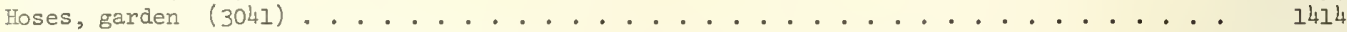

Hose/Socks $(2251,2252,3842)$. . . . . . . . . . . . . . . . . . 1645

Hot Pads, Oven Mits, Potholders (2392) ..................... . . . . . . . . . . . . .

Hot Plates, electric (3634) . . . . . . . . . . . . . . . . . . . . . . . . . . .

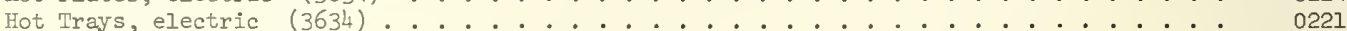

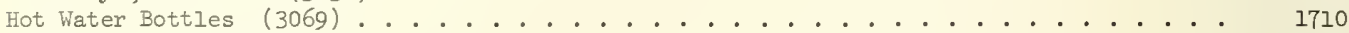

Houses, doll (3944) . . . . . . . . . . . . . . . . . . . . . . . . . 1339

Houses, play and tree houses . . . . . . . . . . . . . . . . . . . . . 1234

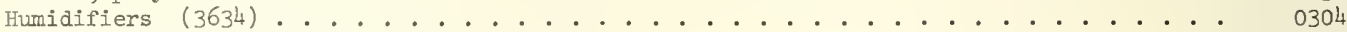

Hygienic personal use items . . . . . . . . . . . . . . . . . . . . . . . . . . 1633

Hypodermic Needles and Syringes (3841) . . . . . . . . . . . . . . . . . 1716

Ice Crackers, manual

Ice Cream Makers, electric (3634)

Ice Cream Makers, manual

Ice Crushers, electric ( 3634 )

Ice Crushers, manual

Ice Makers, electric, separate from refrigerators (3634)

Ice Picks (3423)

Ice Skates (3949)

Ice Skates, hockey (3949)

Igniters, charcoal, electrical

Immersion Water Heaters, electric (3634)

Incinerators, electric (3433)

Incinerators, gas ( 3433$) \cdot \cdot \cdot \cdot \dot{0}$.
Incinerators, not otherwise specified

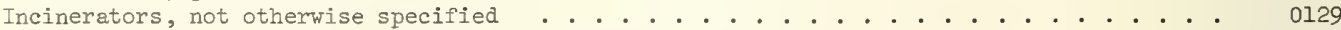

Incinerators, without gas or electric heat supply (3433)... . . . . . . . . . . . 0121

Infant "Carriers," personal use (back packs, slings, etc.) . . . . . . . . . . . . . . 1527

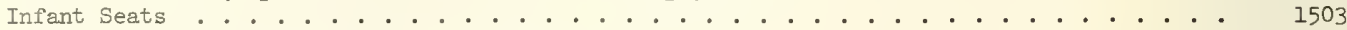

Inflatable Furniture, all kinds... . . . . . . . . . . . . . . . . . . 0659

Inflated Toys, other than balloons (3944) . . . . . . . . . . . . . . . . . . . . 1325

Inflators, tire, hand operated $(3563)$. . . . . . . . . . . . . . . . . . . . . . . . . . . . . . . . . . . . . . . . . . . .

Insect Traps and Insecticide Vaporizers, electric (3699)... . . . . . . . . . . . . 1416

Insecticide Vaporizers, electric $(3634)$. . . . . . . . . . . . . . . . . . 14l6

Instruments, musical, electric $(3931)$. . . . . . . . . . . . . . . . . . . . . . . . . . . . . . . . . 0522

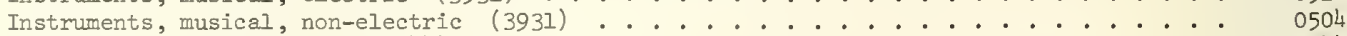

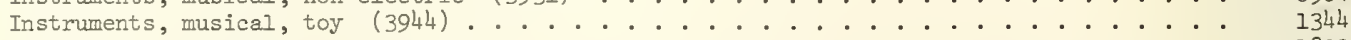

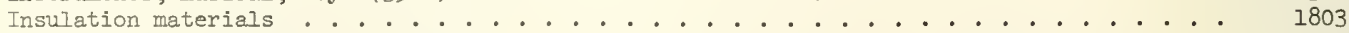

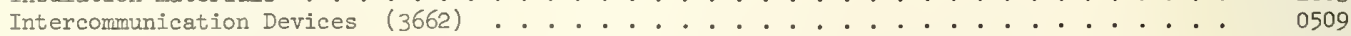

Interrupters, circuit . . . . . . . . . . . . . . . . . . . . . . . . 0705

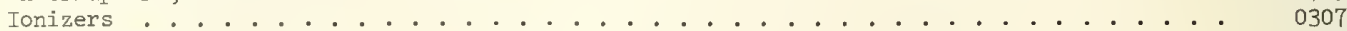

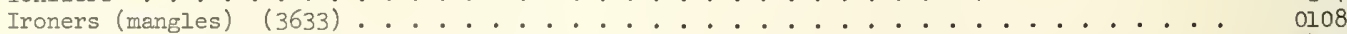

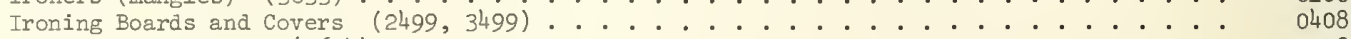

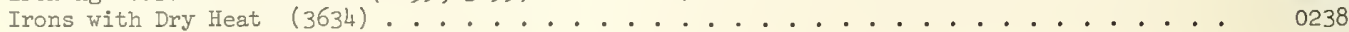


Irons, electric toy (3944)

Irons, not otherwise specified $(3634)$

Irons, soldering (3423)

Irons, toy, not electric (394i)

1321

Jackets $(2328,2329,2337,2339,2363) \ldots \ldots \ldots 1646$

Jacks, Lifts, Hoists $(3423)$. . . . . . . . . . . . . . . . 0814

Jalousie Glass Doors $(2431)$. . . . . . . . . . . . . . . . . . . . 1835

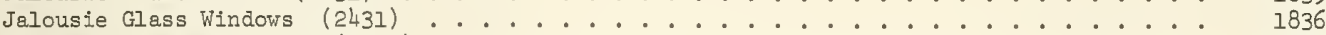

Jars and Bottles, glass (3221) . . . . . . . . . . . . . . . . 1106

Jewelry $(3911,3961) \ldots \ldots \ldots \ldots \ldots 16 \ldots \ldots$

Jewelry, toy $(3944)$

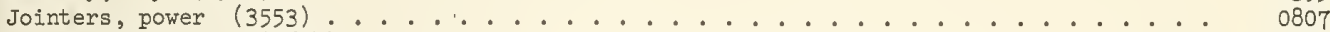

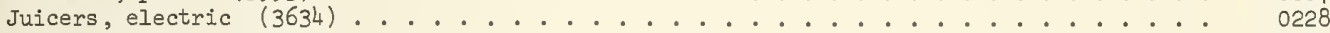

Juicers, manual $(3229)$. . . . . . . . . . . . . . . . . . 0410

Juvenile Sports Equipment $(3949)$. . . . . . . . . . . . . . . . . 1341

$\mathrm{K}$

Kerosene (2911) . . . . . . . . . . . . . . . . . . . . . . . . . . . . . . . . .

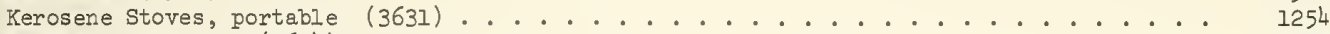

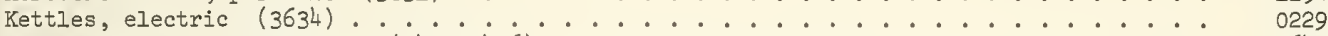

Keys, Key Rings, Key Chains $(3429 ; 3496)$. . . . . . . . . . . . . . . . . . 1643

Kitchen Gadgets; including mixing and measuring spoons/cups, strainers, spatulas, rolling

pins, cake decorators, etc. (3469)

Kites and Kite string (3944)

Kits/Boxes, Tool (excluding tools)

Knick-Knacks, Vases, Statues, Urns

(3469)

$(3079,3229)$

Knife Sharpeners, electric (3634)

Knife Sharpeners, manual (3469)

Knives, electric/battery powered (3634)

Knives, gravity, switchblade, pocket (3421)

0428

1309

0835

0654

0240

0421

0218

Knives, kitchen $(3421,3914)$

1702

0407

I

Lacrosse Equipment (3949)

1215

Ladders $(2499,3499)$

Ladders, chain, for fire escape (3446)

Ladaers, chain, for Laders, swimming pool (3446)

Lamps, electric, table, floor and desk types (3645)

Lamps, gas, installed (3648)

Lamps, heat (364I)

Lamps, gasoline, kerosene and propane (3648)

Lamps/Light Bulbs (3641)

Lamps, sun (3641)

Lanterns, electric (3648)

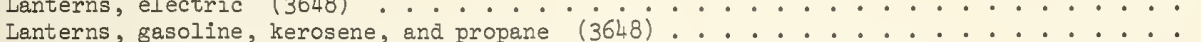

Lapidary Equipment and Tools, including rock tumblers, polishers, etc. . . . . . . .

Lathes, power (3553)

Laundry Baskets (2499)

Laundry Hampers (2499)

Lawn Carts/Wheelbarrows (3524)

Leaf/Grass Catcher Attachments for Mowers $(3524)$

Leaf Mulchers/Grinders, powered, "Shreader-Bagger"

Leashes (3199)

Lenses, contact

License Plates (3469)

Life Preservers $\left(2499,30 \dot{6}^{\circ}, 38 \dot{4}^{\circ}\right)$

$2499,3499)$

18,0619

0703

0636

1231

0626

0625

1634

1226

0627

1609

0639

1226

0533

0805

0438

0672

1425

1431

1433

1715

1624

0821

1220

Lifts

Lifts, other and Elevators (3534)

0814

1814

light Bulbs (3641)

0627

Light Fixtures, electric, attachea (3645)

0628

Lighter Fluids (2899)

Lighter Fuels, charcoal (2899)

Lighters, cigarette/pipe/cigar ( 3634,3999$)$

0940

1247

1604

Lighting Equipment, outaoor (3645)

1410 
Linens, table (2211, 2392)

Lubricants, Machine Oils, Engine Oils (2992).

Luggage Carriers, personal use

Lumber-Boards, Paneling Pieces, not part of structure (2421, 2426, 2491)

Magazines (2721)

Mail Boxes (3469)

Make-Up Kits, toy (3944)

Make-Up Mirrors, lighted

Mangles (3633)

Manicure Devices, electric (3634)

Manicure Sets, not electric

Marbles (3944)

1611

Marker

Masking Tape (2641)

Masks, costume

Masks for Scuba Diving (3949)

Massage Devices, vibrators (3634)

Matches (3999)

Mats, beach (2279, 3069)

Mats, door (2279)

Mattresses and Mattress Covers (2515)

Mattresses, baby crib

Mattresses, camp (3069)

Measuring Spoons (3469)

Meat Grinders, electric (3634)

Meat Skewers (2499)

0230

Mechanical Drawing Toys (3944)

Medicine Cabinets (2514)

Medical Equipment, toys (3944)

Menthol Alcohol, solvent

Metal Polishes/Tarnish Removers/Preventatives (2842)

Meters, electric (3825)

Meters, gas (3824)

Meters, LP gas ( 3824 )

0931

Meters, not otherwise specified

Microscopes (3832)

Microwave Ovens separate from ranges (3631)

Microwave Ovens with ranges (363I)

Mini-Bikes (3751)

Mirrors and Mirror Glass (3231)

Mirrors, lighted make-up

Mittens/Gloves (2381)

Mixers, electric ( 3634 )

Mixing Bowls, Canisters and Similar Containers $(3079,3229$

Mixing Spoons (3469)

Mobile Homes and Related Equipment (245i)

Mobiles, crib

Models and Their Construction Materials (3944)

Models, toy, airplanes and cars, gasoline powered

Molding Sets, metal and plastic (3944)

"Monkey Bars" (3949)........

Mops (2392)

Mops, toy (3944)

Mosquito Netting (2211, 2258)

Moth Balls, Moth Repellants (2879)

Motors, electric, separate (3621) 
Mountain Climbing Equipment, picks, spikes, apparel, etc. (3949) . . . . . . . . 1258

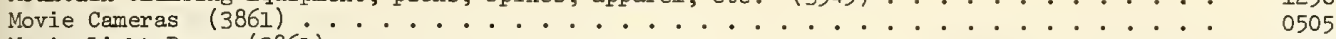

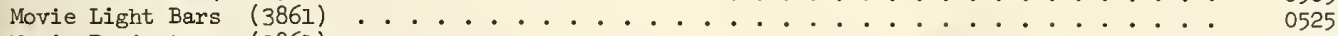

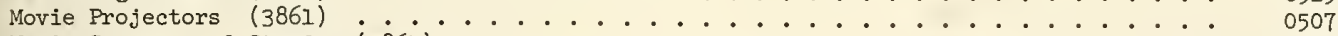

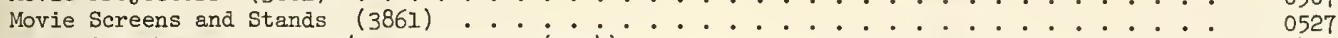

Mower Attachments - grass/leaf catcher (3524) . . . . . . . . . . . . . . . 1431

Mowers, hand $(3524)$. . . . . . . . . . . . . . . . . . 1402

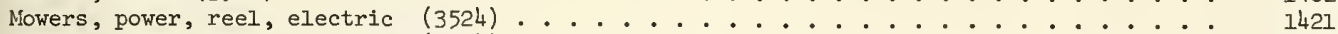

Mowers, power, reel, gasoline $(3524)$. . . . . . . . . . . . . . . . . . 1420

Mowers, power, riding (3524) . . . . . . . . . . . . . . . 1422

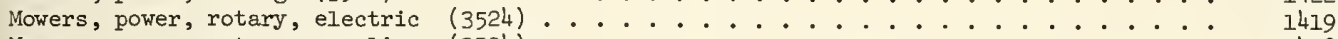

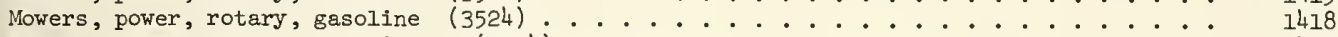

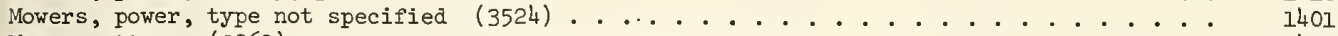

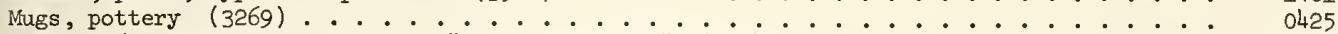

Mulchers/Leaf Grinders, powered, "Shreader-Bagger" (3524) . . . . . . . . . . . 1433

Musical Instruments, electric (3931) . . . . . . . . . . . . . . . . 0522

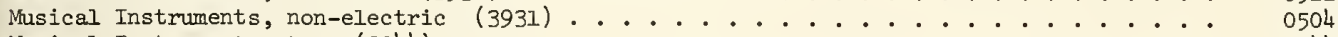

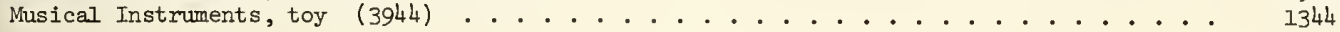

N

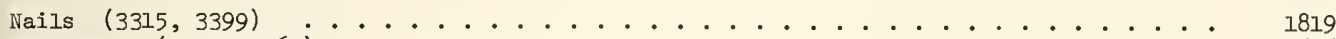

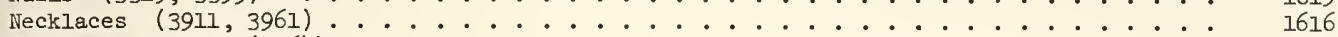

Needles and Pins (3964) . . . . . . . . . . . . . . . . . . . . . . . . . 0918

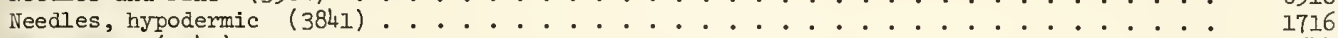

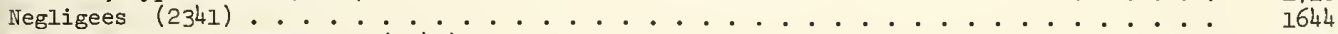

Nets, tennis and badminton (3949) . . . . . . . . . . . . . . . . 1219

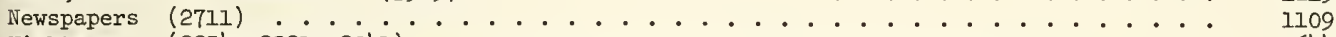

Nightgowns $(2254,2321,2341)$

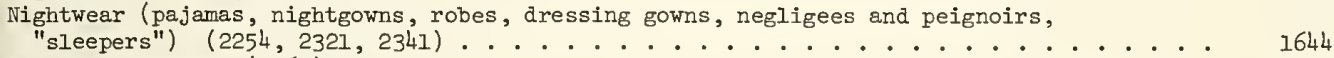

Nipples, Bottles $(3069)$. . . . . . . . . . . . . . . . . . . 1509

Nozzles, garden hose $(3432)$. . . . . . . . . . . . . . . . . 1414

Nursery Furniture and Equipment - other than specified . . . . . . . . . . 1516

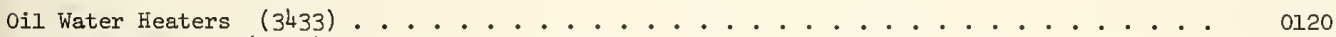

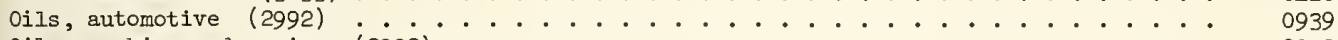

Oils, machine and engine (2992) . . . . . . . . . . . . . . . . . . . . 0913

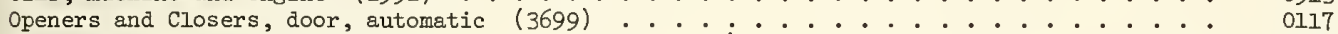

Openers, bottle $(3469,3496) \ldots \ldots \ldots . \ldots \ldots \ldots . \ldots \ldots . \ldots \ldots 22$

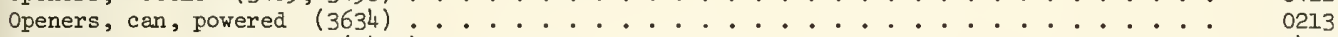

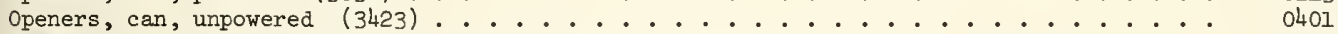

Openers, self-contained, "pop-top" cans, zip-open cans, etc. ........... 1103

Optical Equipment (3832) . . . . . . . . . . . . . . . . . . . 0529

Ornaments, Christmas tree $(3229,3231,3999) \ldots \ldots \ldots \ldots . \ldots \ldots 1713$

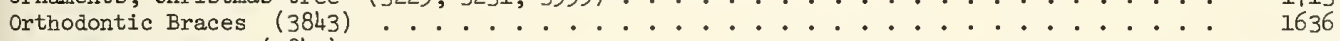

Orthopedic Braces (3842) . . . . . . . . . . . . . . . . . . 1717

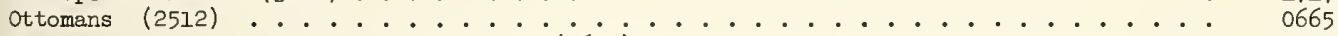

Outdoor Grills, not otherwise specified $(3631) \ldots \ldots \ldots . . \ldots . .259$

Outerwear (coats, raincoats, jackets, snow suits, ponchos, parkas) (2311, 2337) . . . 1646

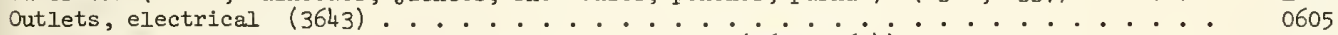

Oven and Range Accessories, racks, broiler pans, etc. $(3631,3634) \ldots \ldots 250$

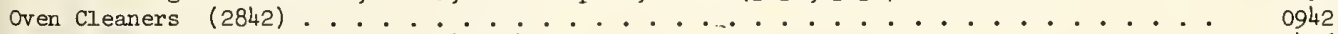

Oven Mits, Hot Pads, Potholders (2392) . . . . . . . . . . . . . . . 0436

Ovens, counter-top, electric $(3634) \ldots \ldots . \ldots 2 . \ldots . . \ldots 216$

Ovens, electric, self-cleaning, separate $(3631) \ldots \ldots . . . \ldots 248$

Ovens, electric, separate from ranges, except self-cleaning $(3631) \ldots . . \ldots 205$

Ovens, electric toy $(3944) \ldots \ldots \ldots \ldots . \ldots \ldots . \ldots \ldots 334$

Ovens, gas, self-cleaning, separate $(3631) \ldots \ldots . . \ldots . . \ldots 249$

Ovens, gas, separate from ranges, except self-cleaning (3631) . . . . . . . . 0206

Ovens, microwave, separate from ranges (3631) . . . . . . . . . . . . . 0207

Ovens, microwave, with ranges $(3631)$. . . . . . . . . . . . . . . 0251

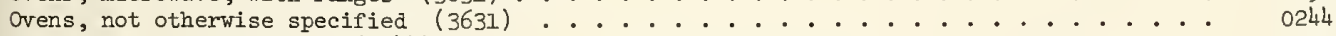

Ovens, toy, not electric $(3944) \ldots \ldots \ldots 1321$

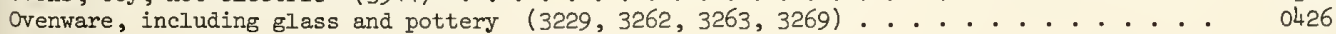


Pacifiers, teething rings (3069)

Pads, bedding, baby

Pads, cleaning

Pails (3079, 3411, 3469)

Pails/Boxes, lunch (3469)

Pails, diaper

Paint and Varnish Removers (285i)
Paint and Varnish Thinners (285i)

Paint Brushes and Rollers (3991)

Paint Sets and Crayons, children

(3944)

Paints, Varnish, Shellac, Rust Preventative, etc. (2851)

Panels, slass, fixed (3231)

Panels, glass, sliding (3231)

Pans and Pots including Lids (3469)

Pans, broiler, oven accessories (3631, 3634)

Pans, fry, electric (3634).

Paper Bags (2621, 2643)

Paper Clips (3496)

Paper Wrapping Products, Paper objects, including towels, tissue, newspapers, stationery,

Patio Lights/Torches, fuel burning

Pillows, water and waterbeds

Ping Pong - table, nets, paddles, balls (3949). 
Pitchers, cream (3079, 3229, 3262, 3263, 3269)

Plant Food fertilizer (2873)

Planters $(3079,3299)$

Plants and Flowers, artificial (3079)

Plants, power, electric, generators (3621)

0606

Plaques and Other Wall Decorative Items (2499, 3079)

0652

Plaster (2899, 3275)

1839

Platters $(3079,3229,3262,3263)$

0418

Play Houses and Tree Houses

1234

Play Tents, children, Play Tunnels and Other Enclosures (3949) . . . . . . . . . . . 1322

Playground Climbing Apperatus, "monkey bars" "grms" (3949)

Playground/Yard Equipment not elsewhere classified (3949) . . . . . . . . . 1201

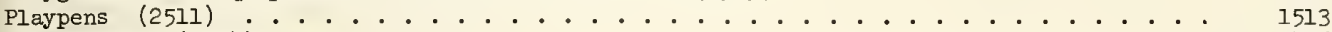

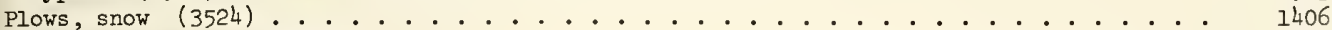

Plugs, electrical (3643) . . . . . . . . . . . . . . . . . . . . . . . . . . . . .

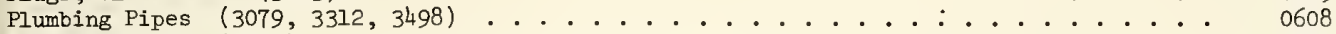

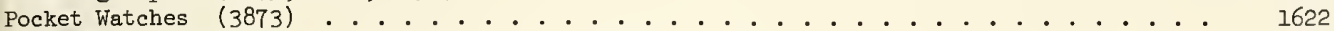

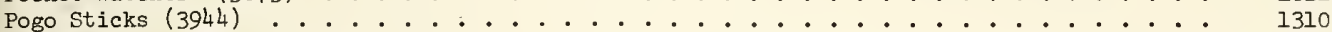

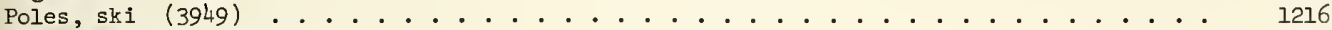

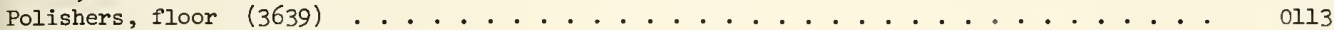

Polishers, shoe, electric $(3634)$. . . . . . . . . . . . . . . . . . 1613

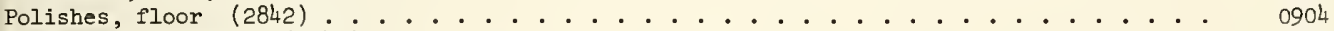

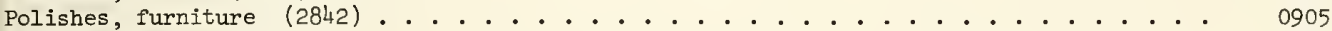

Polishes, metal (2842) . . . . . . . . . . . . . . . . . . . . . . . . . . . . . . . . . . . . .

Polishes, shoe $(2842)$. . . . . . . . . . . . . . . . . . . . 0932

Ponchos (2337) . . . . . . . . . . . . . . . . . . . . . 1646

Pools, swimming - above ground ........................ . . . . . 1262

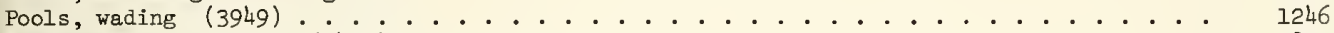

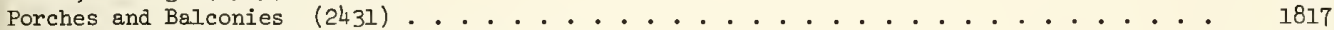

Portable Grills, charcoal, "Hibachi" (3631) . . . . . . . . . . . . . . . . . 1252

Portable Grills, gas (LP) (3631) . . . . . . . . . . . . . . . . . . . 1253

Portable Grills, kerosene (3631) . . . . . . . . . . . . . . . . . . . . 1251

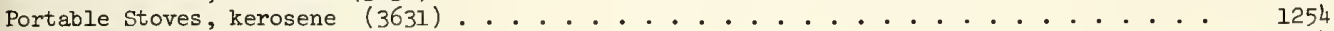

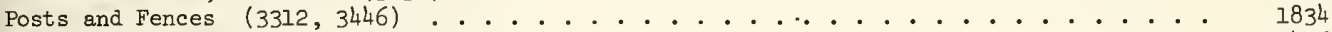

Potholders, Oven Mits, Hot Pads (2392) . . . . . . . . . . . . . . . . 0436

Pots and Pans including Lids $(3469)$. . . . . . . . . . . . . . . 0406

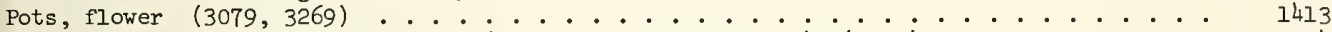

Pottery, Clay and Ceramic Supplies (not including tableware) (3952) . . . . . . 0514

Power Plants, electric/generators (3621) . . . . . . . . . . . . . . . 0606

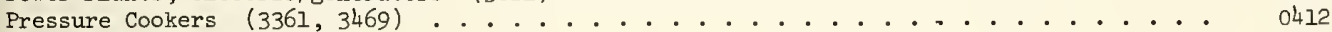

Pressurized Containers ......................... . . . . . . . . . . . . . . . . . . . .

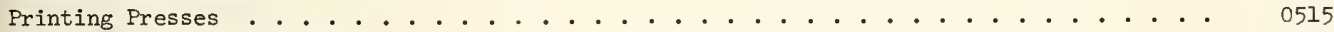

Projectors, movie $(3861)$. . . . . . . . . . . . . . . . 0507

Projectors, slide $(3861)$. . . . . . . . . . . . . . . . . . . . . . . . . . . . . . . .

Pruning and Trimming Equipment, manual (3423) . . . . . . . . . . . . . 1424

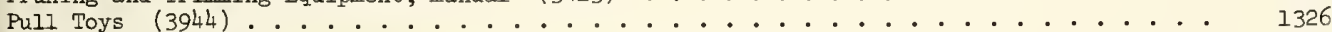

Pumps for Aquariums . . . . . . . . . . . . . . . . . . . . 1240

Pumps, including electric submersible fountain pumps (3561) . . . . . . . . . 1412

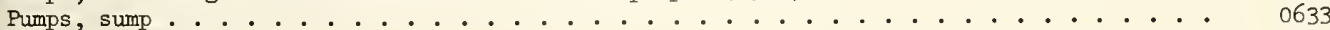

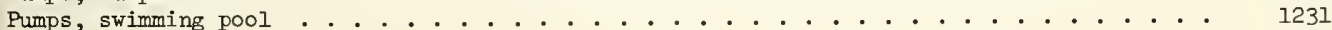

Puzzles $(3944) \ldots \ldots \ldots . \ldots \ldots \ldots$

Q

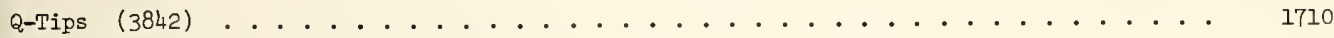

Racquets, tennis, badminton (3949) . . . . . . . . . . . . . . 1219

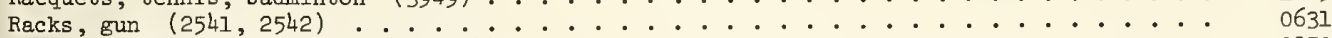

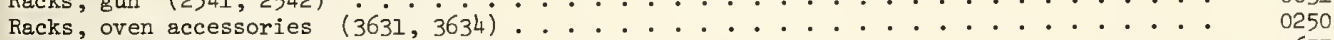

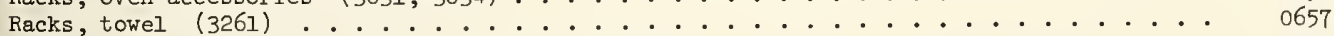

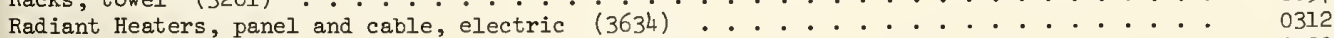

Radiators, household $(3433)$. . . . . . . . . . . . . . . . . 0320

Radios, all models $(3651)$. . . . . . . . . . . . . . . . . . 0502

Railings, Handrails, Banisters $(2431)$. . . . . . . . . . . . . . . . . 1829

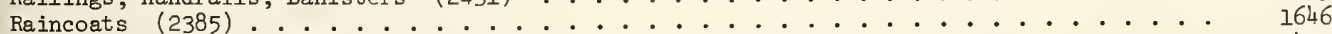

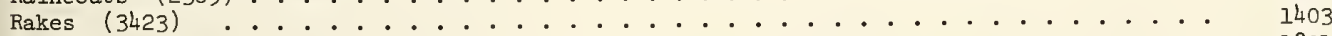

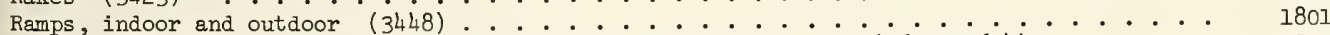

Range and Oven Accessories, including racks, broiler pans, etc. $(3631,3634)$. . . . . 0250

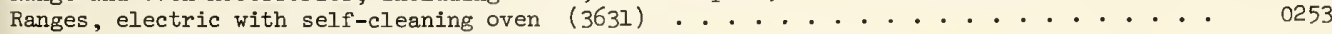


Ranges, gas, with self-cleaning oven (3631)

Ranges, gas, without oven (3631)

\author{
. .
}

Ranges/Stoves, electric with oven, except self-cleaning ( 3631 )

0201

Ranges/Stoves, electric, without oven (3631)

Ranges/Stoves, gas, with oven, except self-cleaning oven (3631)

Ranges/Stoves not otherwise specified (3631)

Rattles, baby

Razors and Shavers $(3421,3634)$

Reamers, manual (3423)

Recliner Chairs (2512)

Record Players, self-contained units (365i)

Recorders, tape and players (3651).

Recording and Reproducting Equipment (3651)

Recording Tapes (3652).

Records, phonograph (3652)

Refrigerators, electric (3632)

Refrigerators, gas (3632)

Refrigerators, not otherwise specified (3632)

Removers, paint and varnish (2851)

Removers, rust (2842)

Removers, spot (2842)

Respiratory Protection Devices

Rewinds, photographic editing equipment ( $38 \dot{6} 1$ )

Riding, Horseback Equipment and Accessories including apparel (3949)

Riding Toys, other, not powered, not including bicycles (3944)

Riding Toys, powered, not including bicycles (3944)

1331

Rifles, cap toy (3944)

1330

Rings (3911, 3961)

Rings/Hooks, drapery (2591)

Rossters, electric (3634)

1616

Robes (2253, 2369, 2384)

0673

0205

Rock Tumblers, Polishers

1644

Rocking Horses and Similar Child Carrying Toys (3944)

Rocketry Sets (3944)

Rockets, cap, toy (3944)

Rocking Chairs (2511)

Rods, curtain and drapery (2591)

Rods, lightning (3643)

Rollers and Brushes, paint (3991)

1327

1314

1348

0671

0655

0706

0925

Rollers, exercise equipment (3949)

Rolling Pins (2499)

Roofs and Roofing Materials (2661, 2952, 3281, 3292, 3444 )

0428

1806

Room Dividers/screens (2541)

Rope and String (2298)

Ropes, tow, for water skiing (2298)

Rotisseries (3634)

Routers, power (3553)

Rubber Bands (3069)

Rug Cleaners/Shampooers, electric (3639)

1626

Rugs, throw type (2272, 2279)

Rulers (2499, 3423)

Runners and Throw Rugs (227i, 2272)

0114

0612

1626

Rust Preventatives (2851)

0612

Rust Removers (2842)

0937

S

Saddle and Pads, horseback riding (3199) . . . . . . . . . . . . . . . . . . . 1239

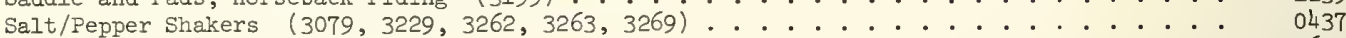

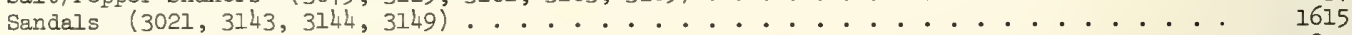

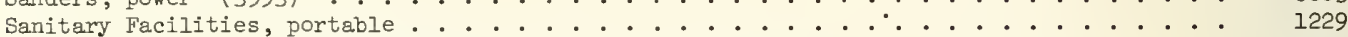

Sanitizing and Cleaning Compounds, solvent-based $(2841,2842)$. . . . . . . . . . . . . 0903

Saucers and Cups $(3079,3229,3263)$.....

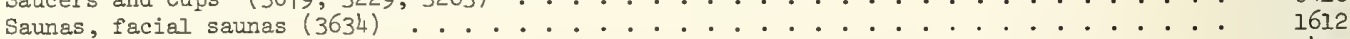

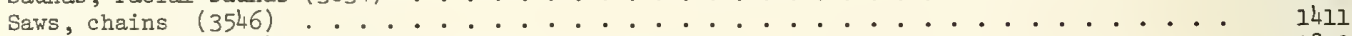

Saws, manual (3425) .. . . . . . . . . . . . . . . . . . . . . . . 0830

Saws, portable, circular, powered $(3546)$. . . . . . . . . . . . . . . . 0832

Saws, power, not otherwise specified $(3546,3553)$. . . . . . . . . . . . . . . . . . 0801

Saws, power, portable not circular, electric $(3546)$. . . . . . . . . . . . . . . 0824

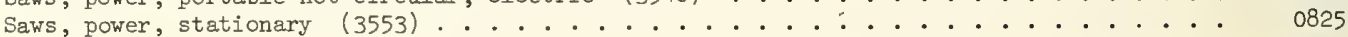


Scissors, electric

Scissors, manual ( $3 \dot{4} 2 i)$

Scooters, motor, unlicensed

Seasonal Decorations, Easter Baskets, Halloween Decorations . . . . . . . . . . . 1714

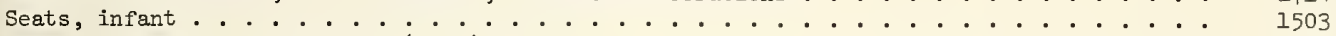

Seats, non-standard, bicycle $(3751)$. . . . . . . . . . . . . . . . 1202

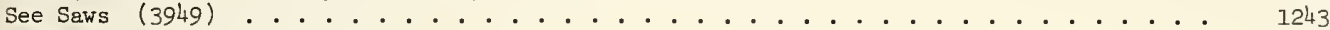

Seed and Fertilizer Spreaders . . . . . . . . . . . . . . . . . . . 1430

Seltzer Bottles (3221) . . . . . . . . . . . . . . . . . . . . . 0439

Septic Tanks, Cesspools and Cisterns $(3079,3272,3443) \ldots 1818$

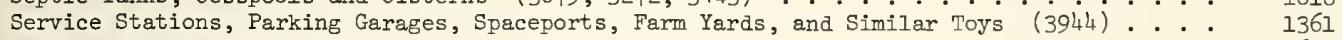

Sewing Basket Articles

Sewing Machines and Accessories (3636)

1632

Sewing Machines, toy (3944)

0112

Shades, window (2591)

Shampooers/Cleaners, rug, electric (3639)

Shapers, power (3541, 3553)

Sharpeners, knife, electric (3634)

Sharpeners, knife, manual (3469).

Shavers and Razors (3421, 3634)

Sheet Metal (3444). - i $\cdot$ il)

1321

0638

0114

0808

0240

0421

1601

Sheets and Pillowcases (22ll)

1833

0614

Sheets, electric ( $363 \dot{4}^{\circ}$

1507

Shellac (2851)....

Shelves and Storage Areas

Shelves, freestanding (2541)

0130

0907

Shields, light shields ( 3851 )

0632

0664

1607

Shirts $(2321,2328,2361)$

Shoe Polishers, electric $(3634)$. . . . . . . . . . . . . . . . . 1613

Shoe Polishes (2842) . . . . . . . . . . . . . . . . . . . . . . . . . . . . . . . . . . . . .

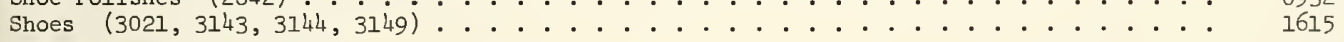

Shoes - see also Footwear

Shovels, garden $(3423)$. . . . . . . . . . . . . . . . . . . 1403

Shovels, snow (3423) . . . . . . . . . . . . . . . . . . . . . . 1415

Shower and Bathtub Enclosures of materials other than glass $(3079,3431) \ldots 0610$

Shower and Bathtub Structures other than doors and panels, including the tub, walls,

handgrips, etc.

Shower Curtains (2392)

0611

0617

Shower Enclosures, glass $(3231)$

"Shreader-Baggers", Leaf Mulcher/Grinder, powered $(3524)$

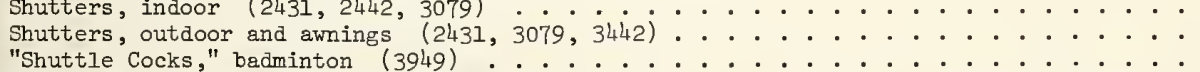

0609

1433

0638

1808

1219

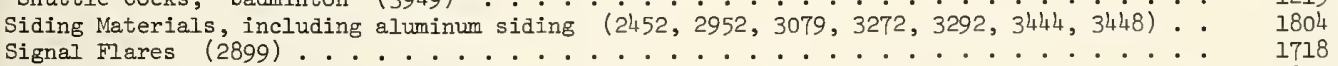

Sills, door and window (243i, $3272,344 \dot{2})$

Sinks (3261)

1831

Sinks, toy $(3944)$

0648

1321

Skateboards $(3944) \ldots \ldots \ldots \ldots . . \ldots \ldots 1333$

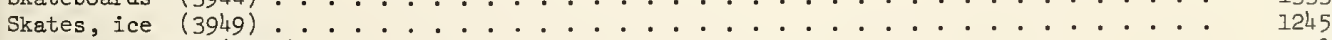

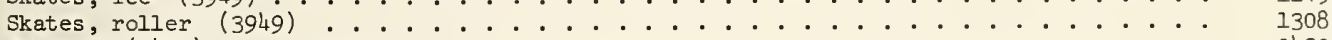

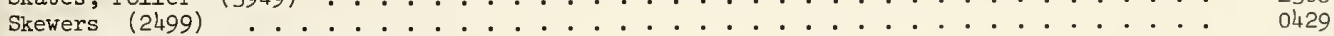

Skiing Equipment, snow $(3949)$. . . . . . . . . . . . . . . . . . 1216

Skillets, electric $(3634)$ 
Skis, water and associated equipment for water skiing (3949) . . . . . . . . . . . . 1264

sleds (3944)

"Sleepers" (23íi)

Sleeping Bags, adult (2399)

Sleeping Bags, children

Slicers/Choppers, electric

Slicers/Graters, vegetable

Slide Projectors (3861)

Slides/sliding Boards (3949)

sliding Boards (3949)

Sliding Panels, glass (3231)

Slingshots, "Sling Propelled" Toys" (3944)

1242

1242

Slip Covers (2392)

Slippers (3142)

1352

0650

Sneakers (3021)

Snorkling Equipment (3949)

1615

Snow "Disks" and similar ite

(3949)

1615

Snow Suits (2363)

Snow Throwers and Snow Plows (3524)

1220

1274

1646

Snowmobiles (3799)

1406

Soap (2841)

Soccer Equipment and Apparel

1218

0901

Sockets, electric (3643)

Socks/Hose (2252)

Sofas and Sofa Beds (2512)

Soft Drink Bottles, glass (2086, 322l)

1267

0605

1645

0647

Softeners and Conditioners, water, appliance (3589)

1110

Soldering Compounds (2899)

Soldering Guns and Irons (2423)

Solvent Based Cleaning and Sanitizing Compounds (2841, 2842)

0125

0833

0813

Sound Recording and Reproducing Equipment (3651)

0903

Space Heaters, electric (3634)

Space Heaters, gas, attached (3433)

Space Heaters, kerosene, attached (3433)

Spaceports, Farm Yards, Service Stations, Parking Garages and Similar Toys (3944)

0503

0313

0314

0315

Spackling/Caulking Compounds (2891)

Spatulas (3469)

Speakers, remote (3651)

Special Equipment for the Injured or Aged, other (3842)

Spectacles (3851)

Spikes, mountain climbing (3315)

Splicers (3861)

Splicers, photographic (3861)

Spools, thread (2499)

Spoons, Forks (3914)

Spoons, measuring and mixing (3469)

Sports Clothing (2329, 2339)
Sports Equipment, Juvenile (3949)

Spot Removers (2842)

Spouts/Drain Pipes

Sprayers, garden (3563)

Sprayers, paint (3563)

Spreaders, fertilizer (3523)

Springs and Frames for Beds (2515)

Springs, baby cribs

Sprinklers, garden (3432)

Squeaker/Squeeze Toys (3069, 3944)

Stairs, Ramps and Handrails, indoors and outdoors (2431, 3446 )

Stakes, garden (2499)

Stakes, tent, camping (2499)

Stands, Christmas tree and Supports (2499)

Stationery (2648, 2753)

Statues, Knick-Knacks, Vases, Urns (3269, 328i) 


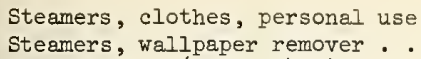

Stepladders (2499, 3499)

Stepstools (2511, 2514)

Stereo Components and Equipment

$(3651)$

Sterilizers, baby bottle (3551)

0620

Sterno (fuel for chafing dishes)

0520

Stilts, toy (3944)

Stools, bar (2511, 2514, 2519)

0941

Stools, Stepstools (25i1, 2514)

1349

Storm Doors, glass (2431)

0658

Storm Windows $(2431,3442)$

0620

stoves, coal (3433)

Stoves, heating, gas (3433)
Stoves, heating, kerosene (3433)

Stoves, heating, not otherwise specified (3433)

1823

1826

0333

0330

Stoves, portable, kerosene (3631)

Stoves/Grills, portable, gasoline, for cooking ( $363 i$ )

0331

0324

Stoves/Ranges, electric, with oven, except self-cleaning $(3 \dot{6} 3 \dot{i})$

Stoves/Ranges, electric, without oven (3631)

Stoves/Ranges, gas, with oven, except self-cleaning oven ( $363 \dot{1}^{\circ}$

1254

1224

0201

Stoves/Ranges, not otherwise specified (3631)

0202

0203

Stoves, table, open flame (3631)

0243

Stoves, toy (3944)

Stoves, wood (3433)

Straight Ladders (2499, 3499)

Strainers, cooking (3469, 3496)

String and Rope (2298)

Strollers (3949)

Strollers, doll (3944)

Sugar Bowls (3079, 3229, 3262, 3263, 3269)

0403

1321

0332

0619

0428

0919

1522

1340

Suitcases (3161)

Suits (2311, 2337)

0437

1623

Sump Pumps (3amps (364l)

1645

0633

Sunglasses (3851)

1609

Surfboards (3949)

1607

1261

1645

Sweaters (2253, 2329)

Sweepers/Brooms, electric $(3635)$. . . . . . . . . . . . . . . . . . . . . . . . . 0116

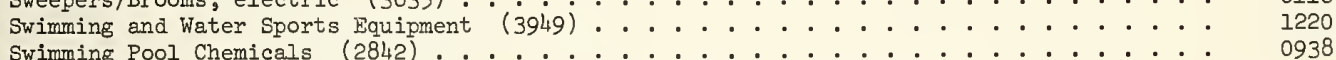

Swimming Pools - above ground, all associated equipment including frame,

filter pumps, etc. . . . . . . . . . . . . . . . . . . . . . . . . . . 1262

Swimming Pools and Associated Equipment (not including above ground pools) . . . . . . 1231

Swiming/Water Toys Designed for Children (3944). . . . . . . . . . . . . . . . 1365

Swings, baby .. . . . . . . . . . . . . . . . . . . . . . . . . . 1521

Swings/Swing Sets (3949) . . . . . . . . . . . . . . . . . . . . . . 1241

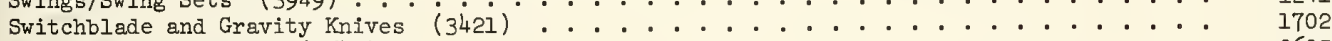

Switches, electrical (3643) . . . . . . . . . . . . . . . . . . . . . . 0605

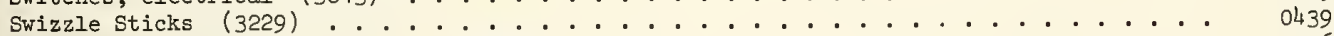

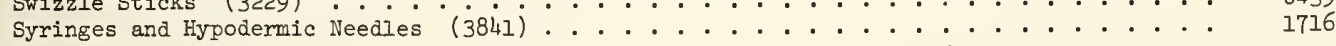

Table Linens $(2211,2392)$. . . . . . . . . . . . . . . . . . . . . 0651

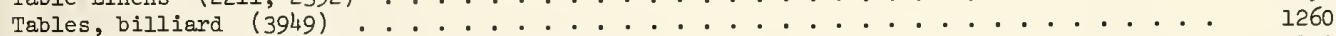

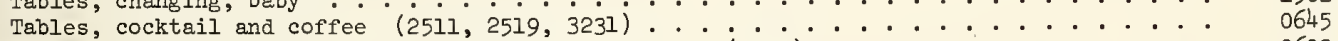

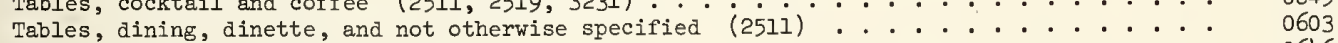

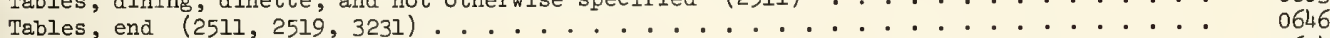

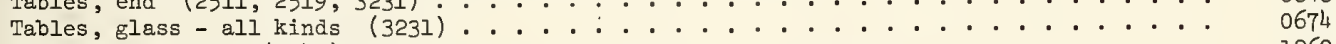

Tables, ping pong (3949) . . . . . . . . . . . . . . . . . . . . . . . 1269

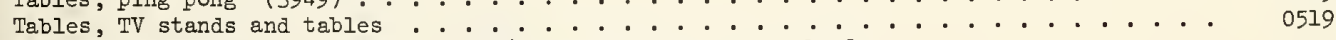

Tableware Accessories, including salt/pepper shakers, sugar bowls,

cream pitchers, etc. $(3079,3229,3262,3263,3269)$. . . . . . . . . . . . . 0437

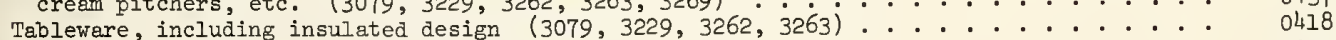

Tackle Box (3949) . . . . . . . . . . . . . . . . . . . . . . 1210

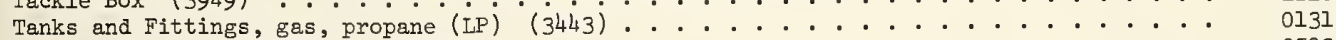

Tape Recorders and Players $(3651)$. . . . . . . . . . . . . . . . . . . . . . . . . 0503

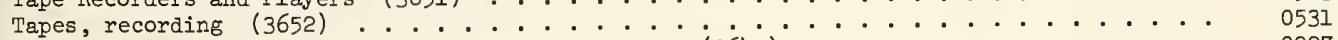

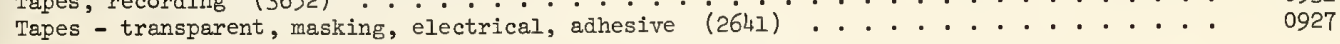


Television sets - see TV

Tennis and Badminton Equipment, including nets and standards, racquets, tennis

balls, "shuttle cocks", etc. (3949)

Tents, play/toy (3949)

Thermometers, cooking (3829)

Thermos Containers (3429)

Thermostats for any Heating/Cooling System (3822)

Thimbles (3469)

Thinners, paint and varnish (2851)

Threads (2284)

Throwers, snow (3524)

0440

0441

1102

0340

Tire Chains and Tire Irons (3496)

Tissue Paper (2621)

Toasters (3634)

Tool Sheds (3448)

Tools, automotive, and accessories

Tools, Fireplace Equipment (3429)

Tools, garden, power - other (3524)

Tools, hand, garden (3423)

1409

Tools, lepidary

Tools, power, portable and stationary - other

Tools, toy (3944)

Tools, workshop, manual, and accessories (3423)

Tools, yard, manual, winter (3423)

1403

0533

0809

1336

0810

Toothbrushes, non-powered (3991)

Toothbrushes, powered (3634)

Toothpicks (2499, 3079)

Torches

Torches/Patio Lights, fuel burning

Toupees, hairpiece (3999)

Tow Ropes for Water Skiing (2298)

Towel Racks and Bars (3261, 3469)

Towels, bath and beach (2259, 2392)

Towels, paper (2621)

Toy Balls, only balls designed for

Toy Boxes or Chests (2511)

Toy Home Equipment and Appliances, including ovens, stoves, sinks, irons,

sewing machines, washing machines, etc. (3944)

1415

1629

1608

0431

0811

1428

1605

1264

0657

Toys - see product catagory series 1500 or under (toy) item

Tracks for Toy Cars (not electric) (3944)

Tractors, garden (3524)

Trailers, camping and Related Equipment (not mobile homes)

Trains and Accessories, electric toy (3944) 
Trays, hot, electric (3634)

Trays, including folding TV trays $(2499,3079,3229,3914) \ldots \ldots 0432$

Tree Houses . . . . . . . . . . . . . . . . 1234

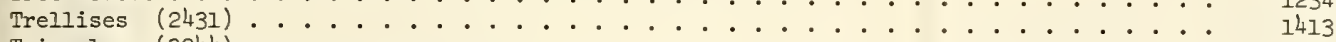

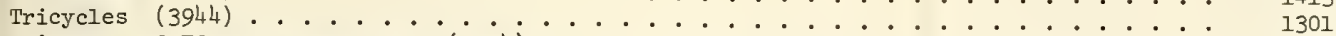

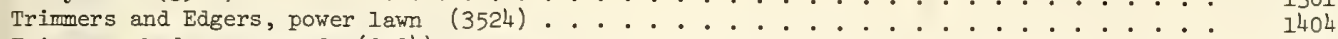

Trimmers, hedge, powered (3524) . . . . . . . . . . . . . . . . 1427

Trimming and Pruning Equipment, manual (3423) . . . . . . . . . . . . . 1424

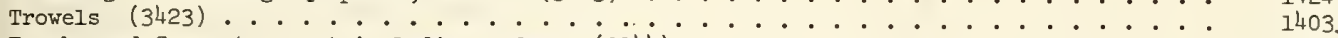

Trucks and Cars, toy, not including models $(3944) \ldots \ldots \ldots . . \ldots \ldots 1305$

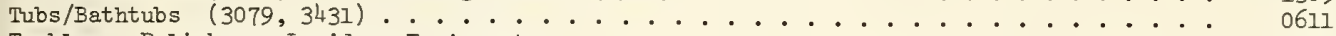

Tumblers, Polishers, Lapidary Equipment . . . . . . . . . . . . . . . . . . . 0533

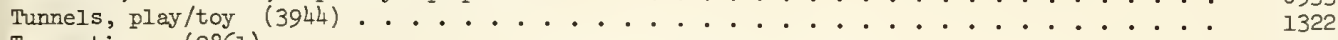

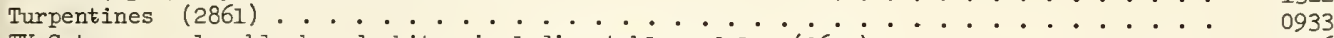

TV Sets, console, black and white, including table models (3651) . . . . . . . . 0516

TV Sets, console, color, including table models (3651) . . . . . . . . . . . . 0528

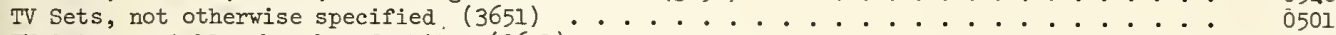

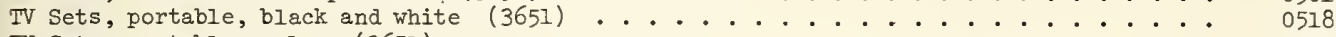

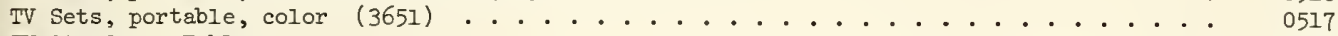

TV Stands or Tables . . . . . . . . . . . . . . . . . . . . 0519

TV Trays (3469) . . . . . . . . . . . . . . . . . . . . . . . . . .

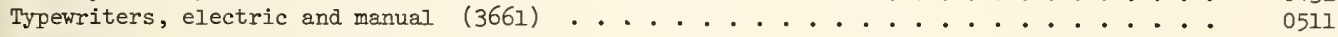

$\mathrm{U}$

Umbrellas, beach (3999) . . . . . . . . . . . . . . . . . . . 1222

Umbrellas, personal use $(3999)$. . . . . . . . . . . . . . . . . . 1621

Urns, Vases $(3229,3269,3281) \ldots \ldots \ldots 654$

Vacuum Cleaners (3635) ....................................... 0115

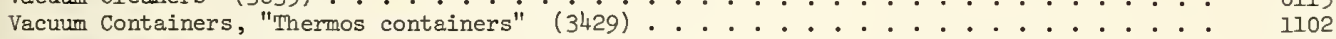

Vacuums, swimming pool . . . . . . . . . . . . . . . . . . 1231

Vaporizers, personal use $(3634) \ldots \ldots \ldots 305$

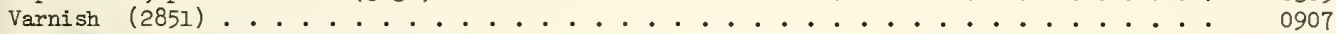

Varnish and Paint Thinners (2851) . . . . . . . . . . . . . . . . . . 0908

Vases, Urns $(3229,3269,3281) \ldots \ldots \ldots \ldots \ldots \ldots \ldots$

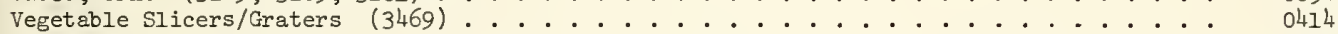

Venetian Blinds (2591) . . . . . . . . . . . . . . . . 0638

Vests $(2311,2329) \ldots \ldots \ldots \ldots 1645$

Viewers, photographic editing equipment (3861) . . . . . . . . . . . . . 0506

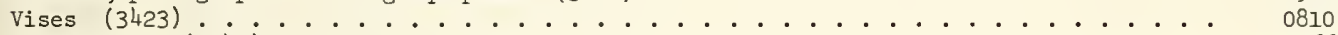

Volleyballs $(3949)$. . . . . . . . . . . . . . . . . . . . . 1266

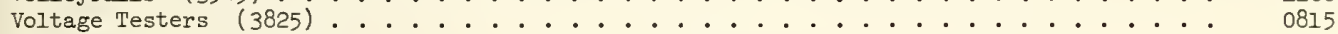

Wading Pools (3949) . . . . . . . . . . . . . . . . . . . . . 1246

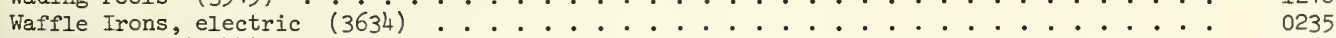

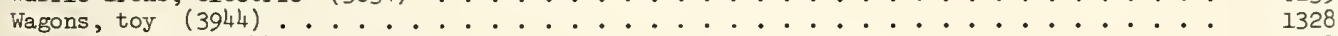

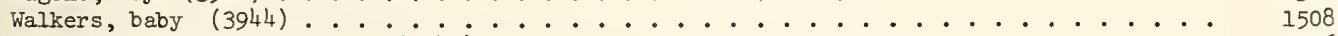

Walkers, Crutches and Canes $(3842) \ldots \ldots \ldots \ldots$. . . . . . . . . . . . . . . . . . . . . . . . .

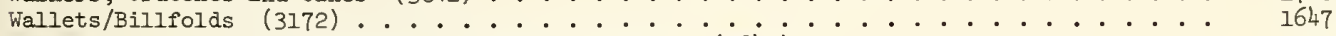

Wallpaper Cleaners and Removers, including steamers $(2842)$. . . . . . . . . . 0922

Walls for Shower and Bathtub Structures (3253) . . . . . . . . . . . . . . 0611

Warmers, bottle $(3079)$. . . . . . . . . . . . . . . . . 1510

Warming Plates $(3634)$. . . . . . . . . . . . . . . . . . . . . . . . . . . . . . . . . . . . .

Wash Tubs . . . . . . . . . . . . . . . . . . . . 0923

Washers - see Washing Machines

Washing Machines, not otherwise specified (3633) . . . . . . . . . . . . . 0126

Washing Machines, toy $(3944)$. . . . . . . . . . . . . . . . . 1321

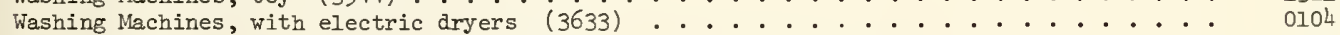

Washing Machines, with gas aryers (3633) . . . . . . . . . . . . 0105

Washing Machines, with spin dryers, without thermal dryers $(3633) \ldots . . \ldots 103$

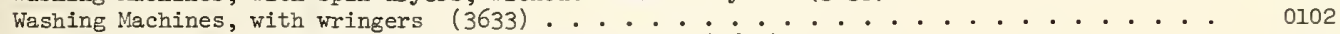

Washing Machines, without wringers or other dryers (3633) . . . . . . . . . . 0101

Waste Containers, trash can/basket, etc. (3469) . . . . . . . . . . . . 0413

Watches, wrist, pendant, pocket (3873) . . . . . . . . . . . . 1622

Wax/Paraffin Candles (3999) . . . . . . . . . . . . . . 0415 
Waxers, floor (3639)

Water Fountains $(3585)$

Water Heaters, electric $(3639)$. . . . . . . . . . . . . . .

Water Heaters, faucet $(3639)$. . . . . . . . . . . . . . . . .

Water Heaters, gas $(3433)$. . . . . . . . . . . . . . . . . .

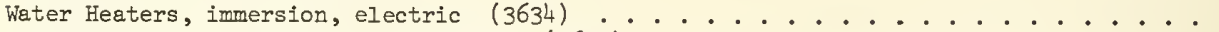

Water Heaters, not otherwise specified (3639)

Water Heaters, oil (3433)

Water Skiing and Associated Equipment, skiis, tow ropes, etc. (3949) . . . . . . . 1264

Water Softeners and Conditioners, appliance $(3589) \ldots \ldots . . . \ldots . . . .$. Water Sports Equipment $(3949)$. . . . . . . . . . . . . . . .

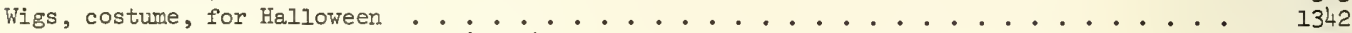

Wigs, Falls and Other Hairpieces (3999) . . . . . . . . . . . . . . 1605

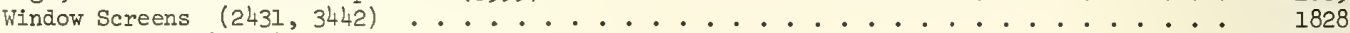

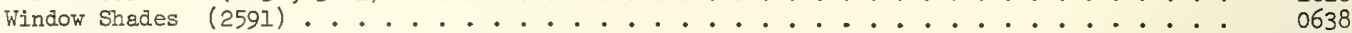

Window Sills, Door Sills, Door Frames, Window Frames $(2431,3442)$. . . . . . . . . 1831

Windows and Window Glass $(2431,3442$ and 3211$) \ldots \ldots \ldots$. . . . . . . . . . . . . . . . .

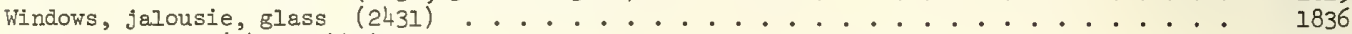

Windows, storm $(2431,3442) \ldots \ldots \ldots \ldots . \ldots \ldots 26$

Windshield Wiper Fluid $(2899)$. . . . . . . . . . . . . . . . 0912

Windup and Battery Operated Toys (not dolls) (3944) . . . . . . . . . 1304

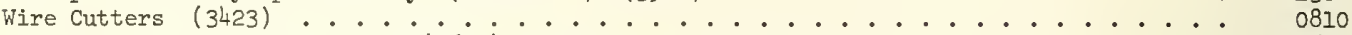

Wire, electrical, replacement (3699) ................ . . . . . . . . . . . . .

Wire, not electric, including picture hanging wire, barbed wire,

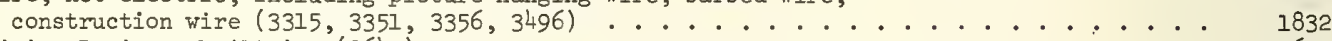

Wiring Devices, built-in $(3643)$. . . . . . . . . . . . . . . . . 0605

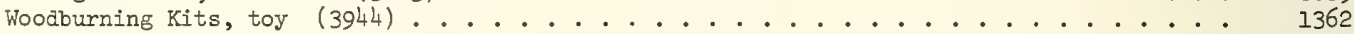

Workshop Compounds/Chemicals, soldering compounds, abrasive compounds . . . . . . . . 0833

Wrapping Products and Containers, aluminum foil (3497) . . . . . . . . . . 1115

Wrapping Products, paper (2621) . . . . . . . . . . . . . 1109

Wrapping Products, plastic, including trash, clothes and garden bags . . . . . . . 1108

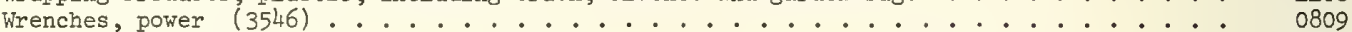

Wrist Watches $(3873) \ldots . . \ldots \ldots . . \ldots \ldots 1622$

\section{Y}

Yard Decorative Equipment, including fish ponds, bird baths, bird houses, mail boxes, etc.

Yard Equipment - see Playground Equipment not elsewhere classified 1201

Yardsticks (2499) 
Product

Number

0101 Clothes Washers Without Wringers or Other Dryers

0102

0103

0104

0105

0106

0107

0108

0109

0110

0111

0112

0113

0114

0115

0116

0117

0118

0119

0120

0121

0122

0123

0124

0125

0126

0127

0128

0129

0130

0131

0201

0202

0203

0204

0205

0206

0207

0208

0209

0210

0211

0212

0213

0214

0215

0216

0217

0218

0219

0220

0221

0222

0223

0224

0225

0226

0227

0228

0229

Washing Machines With Wringers

Washing Machines With Spin Dryers Without Thermal Dryers

Washing Machines With Electric Dryers

Washing Machines With Gas Dryers

Electric Dryers Without Washing Machines Attached

Gas Dryers Without Washing Machines Attached

Ironers "Mangle" (for hand irons see category 0200)

Electric Blankets (for non-electric blankets see 0616)

Electric Heating Pads

Electric Fans, portable (for installed fans see 0341)

Sewing Machines, and Accessories Including Bobbins

Floor Buffers and Waxers

Electric Rug Cleaners/Shampooers (for shampoo see 0901)

Vacuum Cleaners

Electric Sweepers/Brooms (for manual brooms see 0923)

Automatic Door Openers and Closers, Automatic Garage Doors

Gas Water Heaters

Electric Water Heaters

Oil Water Heaters

Incinerators Without Gas or Electric Heat Supply

Electric Incinerators

Gas Incinerators

Water Fountains, with or without cooling or heating units

Water Softeners and Conditioners, Appliance (for chemical softener see 092l)

Washing Machines, not otherwise specified

Clothes Dryers, not otherwise specified

Water Heaters, not otherwise specified

Incinerators, not otherwise specified

Electric Sheets (for non-electric sheets see 0614)

Propane (LP) Gas Taniss and Fittings

Electric Ranges/Stove With Oven, except self-cleaning ovens

Electric Ranges/Stove Without Oven

Gas Ranges With Ovens, except self-cleaning ovens

Gas Ranges Without Ovens

Electric Ovens Separate From Ranges, except self-cleaning

Gas Ovens Separate From Ranges, except self-cleaning

Microwave Ovens Separate From Ranges

Electric Refrigerators

Gas Refrigerators

Electric Freezers, separate

Gas Freezers, separate

Automatic Corn Poppers

Can Openers, powered (for manual can openers see 0401)

Dishwashers

Electric Blenders

Electric Broilers and Grills, Counter-Top Ovens

Electric Coffeemakers and Teapots

Electric/Battery Powered Knives (for manual knives see 0407)

Electric Deep Fryers

Electric Defroster Devices

Electric Food Warmers and Hot Trays

Electric Fry Pans and Skillets

Electric Griddles

Electric Hot Plates

Electric Ice Cream Makers

Electric Ice Crushers

Electric Ice Makers, separate from refrigerators

Electric Juicers

Electric Kettles

0230

Electric Meat Grinders 


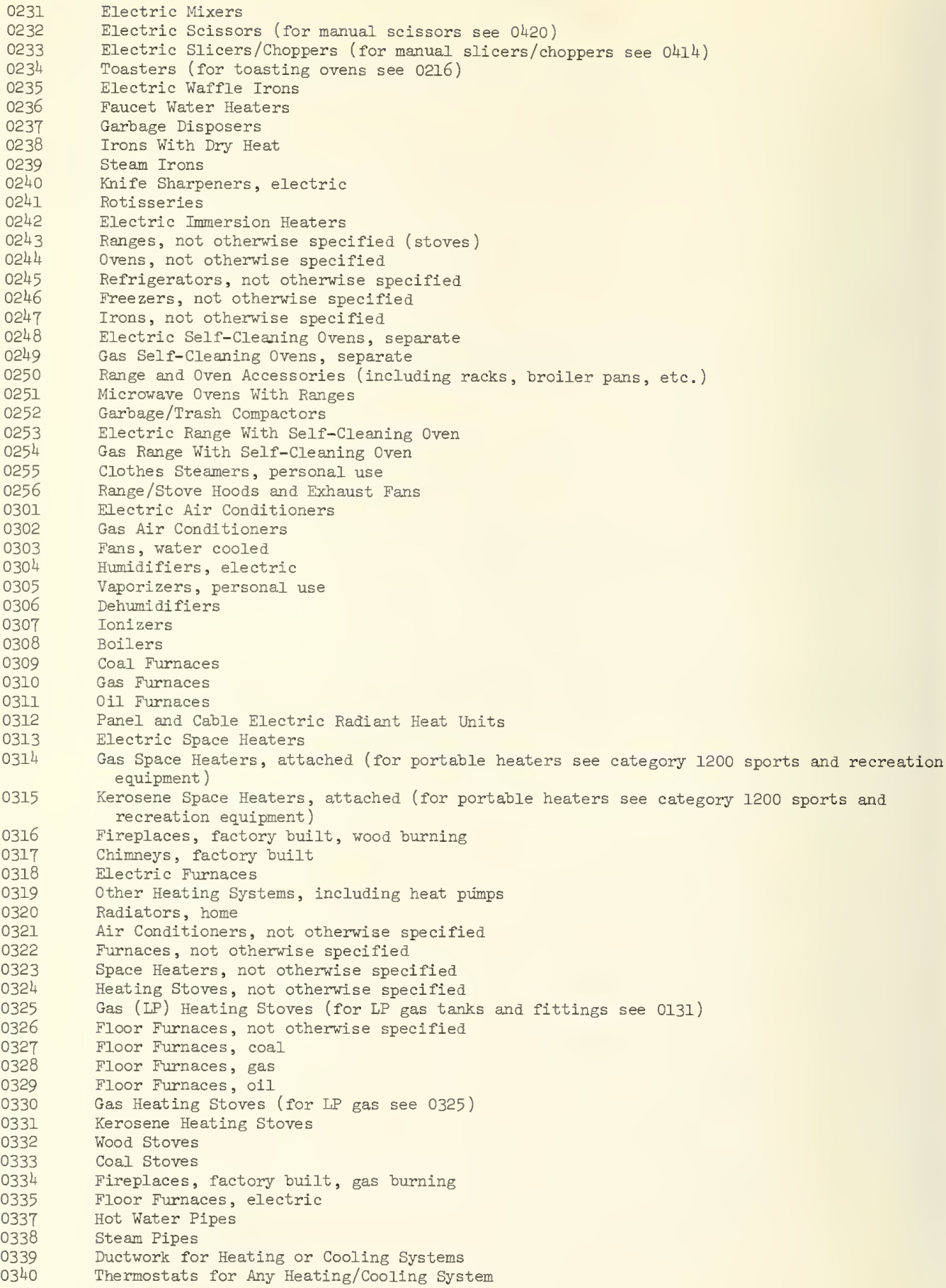


0341 Fans, permanently installed, including attic fans

0401

0402

0403

0404

0405

0406

0407

0408

0409

0410

0411

0412

0413

0414

0415

0416

0417

0418

0419

0420

0421

0422

0423

0424

0425

0426

0427

Can Openers, unpowered, (for powered can openers see 0213)

Chaffing Dishes and Fondue Posts With Open Flame Burners (for fuels see 0941)

Table Stoves, Open Flame

Non-Electric Coffee Grinders

Coffeemakers and Teapots, unpowered

Pots and Pans, including lids

Cutlery, unpowered, "kitchen knives" (for powered cutlery see 02l8)

Ironing Boards and Covers

Manual Ice Crushers

Manual Juicers

Manual Food Grinders

Pressure Cookers and Canners

Waste Containers, Trash Can/Basket, Etc.

Cutting and Chopping Devices Including Vegetable Slicers/Graters (non-electric)

Wax Candles/Paraffin (candleholders see 0433)

Corkscrews

Flatware, except cutlery (spoons, forks)

Tableware, including insulated design (dishes, cups, saucers, serving bowls and platters)

Clothes Hangers, all types, Tie Rack, Belt-Rack, Etc.

Scissors, manual (for electric scissors see 0232)

Knife Sharpeners, manual

Bottle Openers

Butane Candles

Drinking Glasses, glass

Drinking Glasses, non-glass, including plastic, paper cups and pottery mugs

Ovenware, including glass and pottery

Ice Picks

Other Kitchen Gadgets, including mixing and measuring spoons/cups, strainers, spatulas, rolling pins, cake decorators, etc.

0429

0430

0431

0432

0433

0434

0435

0436

0437

0438

0439

0440

0441

0501

0502

0503

0504

0505

0506

0507

0508

0509

0510

0511

0512

0513

0514

0515

0516

0517

0518

0519

0520

0521

0522

Meat Skewers

Ice-Cream Makers, manual

Toothpicks and Hors d'Oeuvres Picks, wood, plastic and metal

Trays including folding TV trays

Candle Holders, candlesticks

Door Stops (not hardware 1820)

Kitchen Mixing Bowls, Canisters and Similar Containers

Potholders, Oven Mits, Hot Pads

Tableware accessories, including salt/pepper shakers, sugar bowls, cream pitchers, etc. Laundry Baskets (for laundry hamper see 0672)

Bar Accessories (for bar stools see 0658) swizzle sticks, seltzer bottles, manual ice crackers, etc.

Thermometers, atmospheric

Thermometers, cooking

Television Sets, not otherwise specified

Radios, all models

Sound Recording and Reproducing Equipment (tape recorders and players)

Musical Instruments, non-electric

Movie Cameras (for film see 0534, for still cameras see 0523)

other Photographic Equipment and Accessories, including editors and splicers (for photographic chemicals see 0926)

Movie Projectors

Slide Projectors

Intercomunication Devices

Telephones and Telephone Accessories

Typewriters, electric and manual

TV and Radio Antennas, indoor (for outdoor see 0526)

Art Supplies and Equipment

Clay, Pottery and Ceramic Supplies and Equipment (not including tableware)

Printing Presses (for home use)

TV Sets, console, black and white including table models

TV Sets, portable, color

TV Sets, portable, black and white

TV Stands or Tables

$\mathrm{Hi}$ Fi and Stereo Components and Equipment, including amplifiers, remote speakers

Record Players, self-contained units

Electric Musical Instruments 
Cameras, still picture and slide (for film see 0534)

0524

0525

0526

0527

0528

0529

0530

0531

0532

0533

0534

0601

0602

0603

Flash Attachments for Cameras, Flash Bulbs

Movie Light Bars

TV and Radio Antennas, outdoor (for indoor see 0512)

Movie Screens and Stands

Television Sets, console, color including table models

Telescopes, Binoculars, Microscopes and Other Optical Equipment

Phonograph Records

Recording Tapes

Glass Bottle Cutting Equipment/Kits

Lapidary Equipment and Tools Including Rock Tumblers, Polishers, Etc.

Film for All Cameras

Beds, including springs, frames (for water beds see 0662, for bedding see 0642)

Chairs, upholstered (see 0644, 0670, 0671)

Tables, dining, dinette, tables not otherwise specified (for end tables, cocktail/coffee tables see 0646 and 0645 , glass top tables see 0674)

0604

0605

0606

0607

0608

0609

0610

0611

0612

0613

0614

0615

0616

0617

0618

0619

0620

0621

0622

0623

0624

0625

0626

0627

0628

0629

0630

0631

0632

0633

0634

0635

0636

0637

0638

0639

0640

0642

0643

0644

0645

0646

0647

0648

0649

0650

Desks, Chests, Bureaus, Dressers, Buffets and Similar Items

Electrical Outlets, Built-In Wiring Devices, and Distribution Systems (for use in or around the household including fuses, fuse boxes; for circuit breakers see 0705)

Electric Power Plants/Generators

Gas Pipes, fittings and distribution systems

Plumbing Pipes

Glass Bathtub and Shower Enclosures

Bathtub and Shower Enclosures of Materials Other Than Glass

Bathtub and Shower Structures Other Than Doors and panels, including the tub, walls, handgrips, etc.

Runners and Throw Rugs, Door Mat

Carpeting, including outdoor carpeting, but excluding runners

Sheets and Pillow Cases

Pillows

Blankets, except electric and baby blankets (bedspreads see 0667)

Drapes, Curtains, including plastic curtains and shower curtains (for rods see 0655; for hooks/rings see 0673)

Stepladders

Straight Ladders

Stepstools

Appliance Cords, Extension Cords and Replacement Wire

Gas Meters

Meters for LP Gas

Electric Meters

Gas Lamps, installed (for portable lamps see 1226)

Electric Table Lamps and Floor Lamps, Desk Lamps

Light Bulbs

Electric Light Fixtures, attached, chandeliers, hanging lamps

Electric Clocks (for non-electric clocks see 0660)

Medicine Cabinets

Gun Cabinets, Ammunition Cabinets and Racks

Cabinets, Shelves and Storage Areas

Sump Pumps

Furniture, not otherwise specified

Fabrics, not otherwise specified

Ladders or Step Stools, not otherwise specified

Meters, not otherwise specified (for household utilities only)

Window Shades and Venetian Blinds, Indoor Shutters (for outdoor shutters see 1808)

Flashlights and Electric Lanterns (for batteries see 0817)

Mirrors and Mirror Glass (for lighted makeup mirrors see 1625)

Bedding, including mattresses, mattress covers and pads

Beach Chairs, Folding Furniture, multiple use, Patio Furniture (for glass top tables see 0674

Chairs, not upholstered or not otherwise specified

Tables, cocktail and coffee tables (for glass top tables see 0674)

End Tables (for glass top tables see 0674)

Couches, Sofas, Sofa Beds, Convertible Sofas, Etc., including cushions

Sinks

Toilets

Slip Covers

Table Linens 
Pictures and Picture Frames, Wall Decorative Items

0653

0654

0655

0657

0658

0659

0660

0661

0662

0663

0664

0665

0666

0667

0668

0669

0670

0671

0672

0673

0674

0701

0702

0703

0704

0705

0706

0707

0801

0802

0803

0804

0805

0806

0807

0808

0809

0810

0811

0812

0813

0814

0815

0816

0817

0818

0819

0820

0821

Artificial Flowers and Plants

Knick-Knacks, Vases, Statues, Urns

Drapery and curtain rods, all kinds

Towel Racks and Bars

Bars and Bar Stools

Inflatable Furniture, all kinds

Clocks, other than electric

Bunk Beds Not Including Bedding

Waterbeds/Water Pillows

Fireplace Equipment, including andirons, screens, tools, grates, non-wood logs

Freestanding Book Shelves, Room Dividers/Screens

Footstools/Ottomans, Hassocks

Bathtowels/Cloths, Beach Towels, Dishtowels/Cloths

Bedspreads, "Throws," Comforters

Books, Magazines, Albums

Clothespins and Similar Fasteners

Recliner Chairs

Rocking Chairs

Laundry Hampers

Drapery Hooks/Rings, Shower Curtain Hooks

Glass Tables - all kinds, indoors and outdoors

Fire Extinguishers

Fire and Smoke Alarms

Fire Escape Devices, Including Chain Ladders

Burglar Alarms

Circuit Interrupters and Circuit Breakers

Lightning Arresters, Rods and Grounding Devices

Locks, Padlocks and Chain Locks

Power Saws, not otherwise specified (see 0824, 0825, 0832)

Power Drills

Power Sanders

Power Routers

Power Lathes

Power Grinders

Power Jointers

Power Shapers

Other Portable and Stationary Power Tools

Workshop Manual Tools and Accessories (chisels, files, vises, wire cutters, pliers, etc.)

Torches

Welding Equipment, electric

Soldering Guns and Irons

Hoists, Lifts, Jacks and Heavy-Duty Chains

Test Equipment, Voltage Testers

Battery Chargers

Batteries, all kinds

Extension Work Lights and Continuous Use Flood Lights

Separate Electric Motors

Internal Combustion Engines, gasoline engine

Automotive Tools and Accessories, Tire Changers, License Plates, Tire Iron (for automotive chemicals see 0939)

Paint Sprayers (for aerosol or spray paint see 1101)

Air Compressors, Separate

Power Saws, portable, not circular, electric

Power Saws, stationary

Power Drill Attachments

Hammers

Screwdrivers

Manual Drills

Manual Saws

Welding Equipment, fuel powered

Powered Portable Circular Saws

Workshop Compounds/Chemicals, Soldering Compounds, Abrasive Compounds

Workshop Staplers, heavy duty

Tool Boxes/Tool Kits (only the box itself, not the tools)

Cleaning Agents and Compounds (soap, cleanser, laundry detergent) 
Bleaches and Dyes, not intended for cosmetic use

Solvent-Based Cleaning and Sanitizing Compounds, including cleaning fluid, spot remover, etc. Waxes, floor

Polishes, furniture

Fumigants

Paints, Varnish, Shellac, Rust Preventative, Etc. (for spray paint see 1101)

Thinners, paint and varnish

Adhesives and Adhesive Products Including Glues

Gasoline

Kerosene

Antifreeze, Windshield Wiper Fluid

Lubricants, Machine Oils, Engine oils

Menthol Alcohol, solvent

Caustics, lye

Charcoal (for charcoal igniters see 1232 and 1247)

Pins and Needles

Rope and String

Caulking/Spackling Compounds

Other Chemicals

Wallpaper Cleaners and Removers, Including Steamers

Cleaning Equipment, Including Brooms, Mops, Brushes and Cleaning Pads, Buckets, Washtubs, Etc. Paint and Varnish Removers

Paint Brushes and Rollers

Photographic Chemicals

Tapes - transparent, masking, electrical, adhesive

Carbon Tetrachloride

Drain Cleaners

Ammonia, household

Metal Polishes, Tarnish Removers/Preventatives

Shoe Polishes

Turpentines

Dishwasher Detergents

Chemical Deodorizers (for aerosol deodorizers see 1101)

Acids, household chemical (muriatic, etc.)

Rust Removers

Swiming Pool Chemicals

Automotive Chemicals (including break fluid, oil, additives, not antifreeze or engine oil)

Lighter Fluid

Fuel for Chafing Dishes, Fondue Pots and Similar Items

Oven Cleaners (for aerosol containers see ll01)

Moth Balls, Moth Repellants

Electric Fences (for non-electric fences see 1834)

Home Pasteurizers

Cream Separators

Pressurized Containers, aerosol cans

Vacuum Containers, "thermos containers"

Self-Contained Openers, "pop top" cans, zip-open cans, etc.

Resealable Closures

Chila Resistant Closures

Glass Bottles and Jars, except soft drink bottles

other Containers, except vacuum or pressure containers

Plastic Wrapping Products, including plastic trash, clothes and garden bags

Paper Wrapping Products, Paper Objects, including paper towels, tissue, newspapers, stationery, etc.

Glass Soft Drink Bottles

Containers, Plastic

Containers, metal (cans)

Oven Cooking/Roasting Bags

Cardboard Boxes, Cartons and Other Cardboard Products

Aluminum Foil Wrapping Products

Playground/Yard Equipment Not Elsewhere Classified, including sandboxes, simulated forts, towns, spaceports, etc. (see 1241 - 1244)

Bicycles and Bicycle Equipment, including "add-on" features such as baskets, horns, non standard seats, handlebars (for infant carriers see 1531)

Boats, Motors, and Accessories for Recreational Use Only, includes sailboats, rowboats, motorboats, etc.

Baseball Equipment, Apparel, Baseballs, Bats "Bases," Masks and Other Protective Gear, Etc. 
Basketball Equipment

1206

Bowling Equipment

1207

Boxing Equipment

1208

1209

Croquet Equipment

Exercise Equipment, including exercise bikes, weights, boards, rollers, etc.

1210

Fishing Equipment, Poles, Lines, Lures, Hooks, Fishing Knives, Scalers, Nets

1211

1212

1213

Football Equipment, including apparel and footware, protective gear, football "kicking tees,"

blocking sleds, tackling sleds, tackling and blocking dunmies, goalposts

1214

1215

1216

1217

1218

1219

1220

Golf Equipment Excluding Carts, including golf balls, clubs, bags, shoes

Golf Carts

Hockey Equipment, including apparel and protective gear, and all equipment

Lacrosse Equipment

Snow Skiing Equipment, skis, poles, boots, etc. (for water skiing see 1264)

Sleds (for snow "disks" see 1274)

Snowmobiles

Tennis and Badminton Equipment, including nets and standards, racquets, tennis balls, "shuttle cocks," etc.

Swimming and Water Sports Equipment, including life preservers, snorkling equipment (for children's water toys see 1365, for scuba gear see 1275)

1221

1222

1223

1224

1225

1226

1227

1228

1229

Headgear for Cycling - Helmets

Beach Equipment, including umbrellas, mats, etc. (for chairs see 0643)

Stationary Outdoor Grills - Kerosene (also see 1249 and 1250)

Portable Gasoline Cooking Stoves/Grills (also see 1251, 1252, 1253, 1254)

Portable Gasoline Heating Equipment (also see 1255 and 1256, for attached/permanent heating devices see category 0300)

Gasoline, Kerosene and Propane Lanterns and Lamps (for installed lamps see 0625)

Battery Powered Cooking Devices

Picnic Equipment, including picnic baskets, coolers, etc.

Camping Equipment, Tents, Tent Stakes, Cots, Camp Mattresses, Adult Sleeping Bags, Hammocks, Mosquito Netting, Portable Sanitary Facilities (for children's sleeping bags see 1360)

1230

1231

Swimming Pools and Associated Equipment, not including above ground pools (1262). Equipment includes filters, pool vacuums, pumps, ladders, diving boards (for wading pools see 1246 , for above-ground pools see 1246, for pool chemicals see 0938)

Charcoal Igniters, electrical (for chemical igniters see 1247)

Trampolines

Play Houses, Tree Houses

Archery Equipment, Bows, Arrows, Targets, Etc.

Unlicensed Motor Scooters and Go-Carts

Gas, Air and Spring Operated Guns Including B-B Guns

Specialized Clothing for Camping or Sports, not elsewhere classified

Horseback Riding Equipment and Accessories, including saddles, pads, bridles, apparel, etc. Aquariums and Accessories, including pumps, heaters, lights, filters, etc.

Swings/Swing sets

Slides

See Saws

Playground Climbing Apparatus, "monkey bars," "gyms"

Ice Skates

Wading Pools

Charcoal Lighter Fuel

Darts and Dart Games, Lawr Darts, Targets, Etc.

Stationary "Built-In" Grills, charcoal

Stationary "Built-In" Grills, gas

Portable Grills, kerosene

Portable Grills, charcoal "Hibachi" (for charcoal see 0917)

Portable Grills, gas (IP)

Portable Stoves, kerosene

Portable Alcohol Heating Equipment

Portable Gas (LP) Heating Equipment

Horseshoes

Mountain Climbing Equipment, including picks, spikes, apparel, etc. (for rope see 0919)

Outdoor Grills, not otherwise specified

Billiards, Tables, Balls, Cues, Chalk, including "pool table"

1261 Surflboards

1262

Swimming Pools - Above Ground and All Associated Equipment, including frame, filter pump, etc. (for pool chemicals see 0938) 


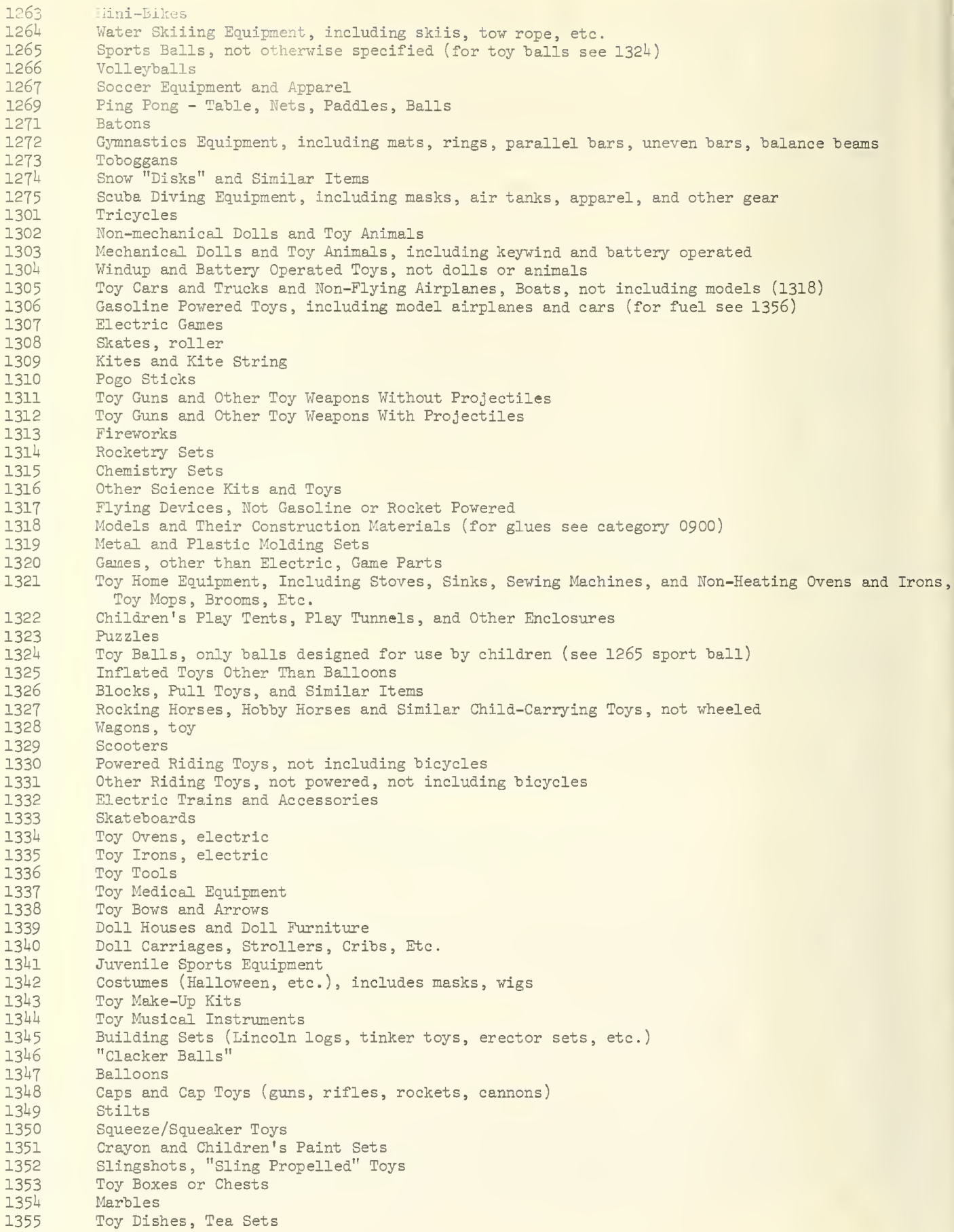


Fuel for Model Cars, Airplanes, Etc.

Mechanical Drawing Toys

Children's Blackboards and Easels

Toy Jewelry

Children's Sleeping Bags

Toy Parking Garages, Service Stations, Spaceports, Farm Yards and Similar Toys

Woodburning Kits

Whistles

Doll Clothes and Accessories

Water/Swimming Toys Designed for Children (for life preservers see 1220)

Children's Books

Toy Banks

Tracks for Toy Cars, not electric

Power Mowers, type not specified (see 1418 - 1422)

Hand Mowers

Hand Garden Tools, including rakes, hoes, trowels, garden shovels, etc.

Power Lawn Trimmers and Edgers

Garden Tractors

Snow Throwers and Snow Plows

Garden Sprayers

Power Tillers and Cultivators (not farm equipment)

1409

1410

1417

1412

Other Power Garden Tools

Outdoor Lighting Equipment, electric

Chain Saws

Pumps, including electric submersible fountain pumps

1413

1414

1415

1416

1417

1418

1419

1420

1421

1422

1423

1424

1425

1426

1427

1428

1429

1430

1431

1432

1433

1501

1502

1503

1504

1505

1506

1507

1508

1509

1510

1511

1512

1513

1514

1515

1516

1517

1518

1519

Greenhouse and Gardening Supplies, including stakes, flower pots, flats, planters, heaters, trellises

Garden Hoses, Nozzles, and Sprinklers

Winter Manual Yard Tools, Snow Shovels, Scrapers

Insect Traps and Insecticide Vaporizers, electrically operated

Yard Decorative Equipment, including fish ponds, bird baths, bird houses, mail boxes, etc.

Power Mowers, rotary, gasoline

Power Mowers, rotary, electric

Power Mowers, reel, gasoline

Power Mowers, reel, electric

Power Mowers, riding

Manual Edgers

Manual Pruning and Trimming Equipment

Wheelbarrows/Lawn Carts

Hatchets, Axes

Powered Hedge Trimmers

Patio Lights/Torches, fuel burning

Plant Food, Fertilizer (for farm use see category 1000)

Seed and Fertilizer Spreaders

Grass/Leaf Catcher Attachments for Mowers

Animal Traps

Powered Leaf Mulchers/Grinders, "Shreader-Bagger"

Highchairs

Changing Tables

Infant Seats

Cribs, including springs and mattresses (for portable cribs see 1529)

Carriages

Gates

Baby Blankets, Sheets, Pads, Pillows, Etc. and Other Baby Bedding Equipment

Walkers, Baby

Bottles, Nipples

Bottle Warmers

Sterilizers

Diapers, including dispossble diapers

Playpens

Baby Baths

Baby Scales

Other Nursery Furniture and Equipment

Baby Rattles

Youth Chairs

Car Seats

1520 Baby Exercisers 
Product

Number

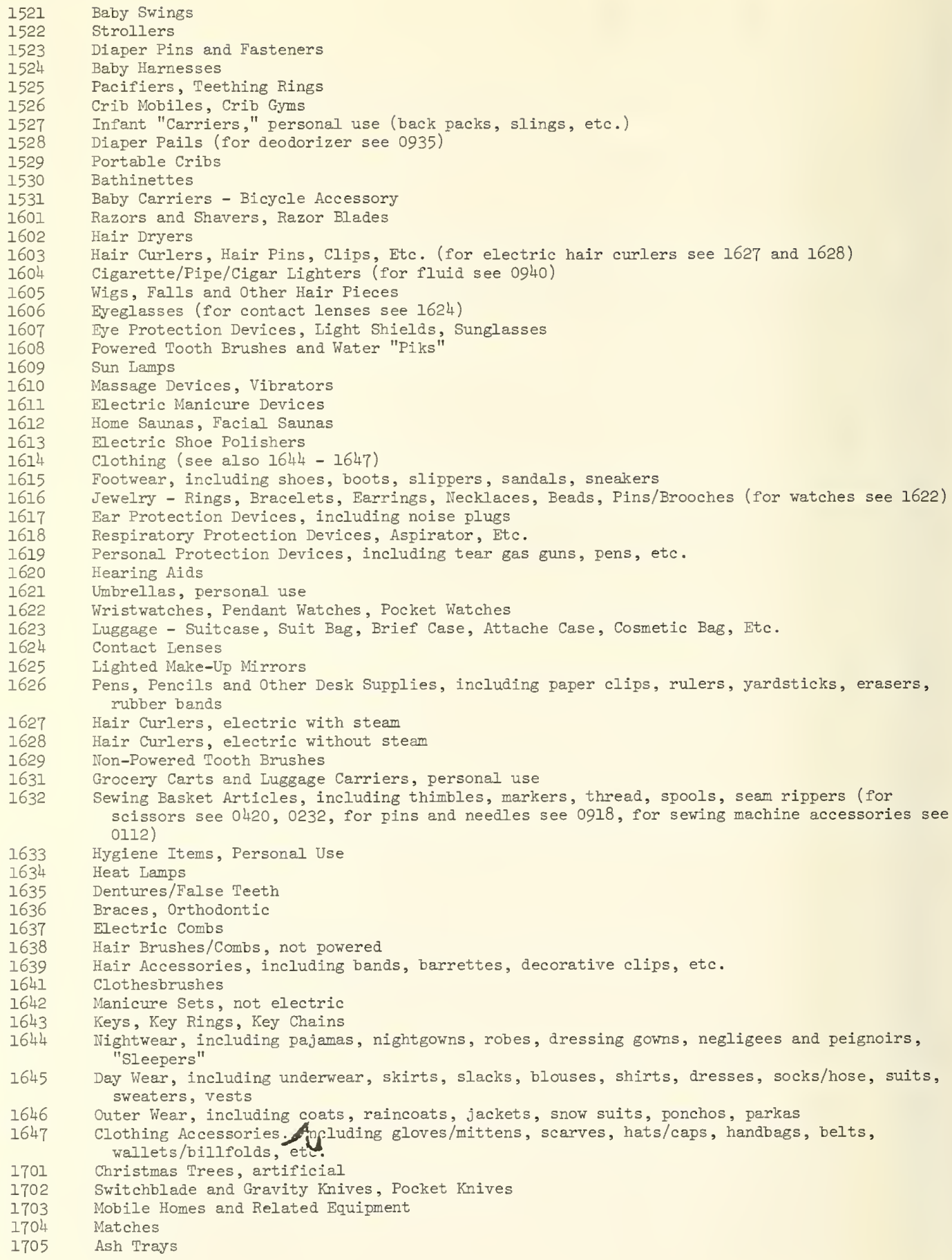

Baby Swings

Strollers

Diaper Pins and Fasteners

Baby Harnesses

Pacifiers, Teething Rings

Crib Mobiles, Crib Gyms

Infant "Carriers," personal use (back packs, slings, etc.)

Diaper Pails (for deodorizer see 0935)

Portable Cribs

Bathinettes

Baby Carriers - Bicycle Accessory

Razors and Shavers, Razor Blades

Hair Dryers

Hair Curlers, Hair Pins, Clips, Etc. (for electric hair curlers see 1627 and 1628)

Cigarette/Pipe/Cigar Lighters (for fluid see 0940)

Wigs, Falls and Other Hair Pieces

Eyeglasses (for contact lenses see 1624)

Eye Protection Devices, Iight Shields, Sunglasses

Powered Tooth Brushes and Water "Piks"

Sun Lamps

Massage Devices, Vibrators

Electric Manicure Devices

Home Saunas, Facial Saunas

Electric Shoe Polishers

Clothing (see also 1644 - 1647)

Footwear, including shoes, boots, slippers, sandals, sneakers

Jewelry - Rings, Bracelets, Earrings, Necklaces, Beads, Pins/Brooches (for watches see 1622)

Ear Protection Devices, including noise plugs

Respiratory Protection Devices, Aspirator, Etc.

Personal Protection Devices, including tear gas guns, pens, etc.

Hearing Aids

Umbrellas, personal use

Wristwatches, Pendant Watches, Pocket Watches

Luggage - Suitcase, Suit Bag, Brief Case, Attache Case, Cosmetic Bag, Etc.

Contact Ienses

Lighted Make-Up Mirrors

Pens, Pencils and Other Desk Supplies, including paper clips, rulers, yardsticks, erasers, mabber bands

Hair Curlers, electric with steam

Hair Curlers, electric without steam

Non-Powered Tooth Brushes

Grocery Carts and Iuggage Carriers, personal use

Sewing Basket Articles, including thimbles, markers, thread, spools, seam rippers (for scissors see 0420, 0232, for pins and needles see 0918, for sewing machine accessories see 0112)

Hygiene Items, Personal Use

Heat Iamps

Dentures/False Teeth

Braces, Orthodontic

Electric Combs

Hair Brushes/Combs, not powered

Hair Accessories, including bands, barrettes, decorative clips, etc.

Clothesbrushes

Manicure Sets, not electric

Keys, Key Rings, Key Chains

Nightwear, including pajamas, nightgowns, robes, dressing gowns, negligees and peignoirs, "Sleepers"

Day Wear, including underwear, skirts, slacks, blouses, shirts, dresses, socks/hose, suits, sweaters, vests

Outer Wear, including coats, raincoats, jackets, snow suits, ponchos, parkas

Clothing Accessories. Apluding gloves/mittens, scarves, hats/caps, handbags, belts, wallets/billfolds, ett.

Switchblade and Gravity Knives, Pocket Knives

Mobile Homes and Related Equipment

Matches

Ash Trays 
Crutches, Canes and Walkers

1707

1708

Wheelchairs

Special Beds

1709

1710

Other Special Equipment for the Injured or Aged

Home First-Aid and Health Equipment, including hot water bottles, thermometers, Q-tips, bandages (not medications)

1711

1712

1713

1714

1715

1716

1717

1718

1719

1720

1721

1801

1802

1803

1804

1805

1806

1807

1808

1809

1810

1811

1812

1814

1815

1816

1817

1818

1819

1820

1821

1822

1823

1824

1825

1826

1827

Christmas Tree Lights

Christmas Tree Stands and Supports

Christmas Tree Ornaments and Other Decorations

Other Seasonal Decorations, Easter Baskets, Halloween Decorations, Etc.

Pet Supplies, Pet Cages, Pet Carriers, Leashes, Collars, Etc.

Hypodermic Needles and Syringes

Braces, Orthopedic

Fmergency Flares, Signal Flares

Lunch Boxes/Pails

Party Favors

Adult Games and Novelty Items

Stairs, Ramps and Landings, indoors and outdoors

Fireplaces, individually built

Insulation Materials

Siding Materials, including aluminum siding

Other Doors, including swinging doors, folding doors, "tract" doors, panel doors, hatches, trap doors, etc. (for glass doors see 1823, 1824, 1825, 1835, 1837, for car doors see 1901) Roofs and Roofing Materials

Floors and Flooring Materials

Awnings and Shutters, outdoor

Covers, patio and porch

Outside Structures, including retaining walls, patios, terraces

Bricks, Concrete Blocks, not part of structure

Gutters, Drain Pipes/Spouts, Run-Off Pipes

Elevators and Other Lifts

Windows and Window Glass, other than storm windows (for car windows see 1901)

Scaffolding

Porches, Balconies

Cisterns, Cesspools and Septic Tanks

Nails, Carpet Tacks and Screws, Thumbtacks

Hardware, including doorknobs, hinges, cabinet pulls, door springs, etc.

Clothes Lines and Collapsible Dryers

Garage Doors

Glass Storm Doors

Glass Panels, fixed

Glass Panels, sliding

Storm Windows

Screen Doors

Window Screens

Handrails, Railings and Banisters

Separate Garages, Tool Sheds, Dog Houses, and Other Pet Enclosures

Window Sills, Door Sills, Door Frames, Window Frames

Wire, not electric, including picture hanging wire, barbed wire, construction wire

other Construction Materials, Weather Stripping, Sheet Metal

Fences, not electric, outdoor, all types, including posts

Jalousie Glass Doors

Jalousie Glass Windows

Glass Doors, not otherwise specified

Lumber, Boards, Paneling, Pieces, not part of structure

Plaster 


\subsubsection{Alphabetical by Acronym}

AA

AAMA

AATCC

ABFLO

$A B Y C$

ACI

AFTMA

AGA

AHAM

AIMA

ALCA.

AMCA

AMO

ANSI

AOAC

ARI

ASHRAE

ASME

ASSE

ASTM

AVATI

AWI

AWPA

AWPB

AWWA

BCA

BCI

BHMA

BIA

BMA

CAGI

CCPI

CEE

CI

CLFMI

CMI

CSMA

CTFA

EEI

EIA

FHDA

FMS

GA

GTA

HAIC

HPMA

IAPMO

IEC

IEEE

IFI

IHF

IIA

ISO

MCAA

MEMA

MIC

MIMA

NAAMM

NAFM

NAPF

NAR

NBHA

NCMA.

NCRPM

NEMA

The Aluminum Association

Architectural Aluminum Manufacturers Association

American Association of Textile Chemists and Colorists

Association of Bedding and Furniture Law Officials

American Boat and Yacht Council, Inc.

American Concrete Institute

American Fishing Tackle Manufacturers Association

American Gas Association

Association of Home Appliance Manufacturers

Acoustical \& Insulating Materials Association

American Leather Chemists Association

Air Moving and Conditioning Association, Inc.

Archery Manufacturers Organization

Amèrican National Standards Institute, Inc.

Association of Official Analytical Chemists

Air-Conditioning and Refrigeration Institute

American Society of Heating, Refrigerating and Air-Conditioning Engineers

American Society of Mechanical Engineers

American Society of Sanitary Engineering

American Society for Testing and Materials

Asphalt and Vinyl Asbestos Tile Institute

Architectural Woodwork Institute

American Wood-Preservers' Association

American Wood Preservers Bureau

American Water Works Association, Inc.

Billiard Congress of America

Battery Council International

Builders Hardware Manufacturers Association

Boating Industry Association

Bicycle Manufacturers Association

Compressed Air and Gas Institute

Composite Can and Tube Institute

International Commission on Rules for the Approval of Electrical Equipment

Cordage Institute

Chain Link Fence Manufacturers Institute

Can Manufacturers Institute, Inc.

Chemical Specialties Manufacturers Association

Cosmetic, Toiletry \& Fragrance Association, Inc.

Edison Electric Institute

Electronic Industries Association

Fir and Hemlock Door Association

Factory Mutual System

Gypsum Association

Glass Tempering Association

Hearing Aid Industry Conference, Inc.

Hardwood Plywood Manufacturers Association

International Association of Plumbing and Mechanical Officials

International Electrotechnical Commission

Institute of Electrical and Electronics Engineers, Inc.

Industrial Fasteners Institute

Institute of High Fidelity

Incinerator Institute of America

International Organization for Standardization

Mechanical Contractors Association of America, Inc.

Maple Flooring Manufacturers Association

Motorcycle Industry Council

Metal Ladder Manufacturers Association

National Association of Architectural Metal Manufacturers

National Association of Furniture Manufacturers, Inc.

National Association of Plastic Fabricators, Inc.

National Association of Rocketry

National Builders' Hardware Association

National Concrete Masonry Association

National Council on Radiation Protection and Measurements

National Electrical Manufacturers Association 
National Fire Protection Association

National Hardwood Lumber Association

National Iubrication Grease Institute

National Model Railroad Association, Inc.

National Oak Flooring Manufacturers Association, Inc.

National Particleboard Association

National Paper Box Association

National Soft Drink Association

National Sanitation Foundation

National Swimming Pool Institute

National Terrazzo and Mosaic Association, Inc.

National Woodwork Manufacturers Association

Packaging Institute, Inc.

Paperboard Packaging Council

Pressure Sensitive Tape Council

Paper Stationery \& Tablet Manufacturers Association

Red Cedar Shingle and Handsplit Shake Bureau

Recording Industry Association of America, Ine.

Rubber Manufacturers Association

Society of Automotive Engineers, Inc.

Screen Manufacturers Association

Safety Helmet Council of America

Sealed Insulating Glass Manufacturers Association

Sheet Metal and Air Conditioning Contractors National Association, Inc.

Snell Memorial Foundation

Society of the Plastics Industry, Inc.

Sump Pump Manufacturers Association

Steel Door Institute

Specialty Wire Association

Steel Window Institute

Technical Association of the Pulp and Paper Industry

Underwriters' Laboratories, Inc.

U.S. Department of Commerce

Vacuum Cleaner Manufacturers Association

Water Conditioning Foundation

Water Systems Council

Wood and Synthetic Flooring Institute of America

Western Wood Products Association 
The Aluminum Association

Park Ridge, Illinois 60068

Air-Conditioning and Refrigeration Institute

Arlington, Virginia 22209

Air Moving and Conditioning Association, Ine.

AMCA

30 West University Drive

Arlington Heights, Illinois 60004

American Association of Textile Chemists and Colorists

AATCC

Box 12215

Research Triangle Park

North Carolina 27706

American Boat and Yacht Council, Inc.

$\mathrm{ABYC}$

15 East 26th Street

New York, New York 10010

American Concrete Institute

ACI

Box 4754, Redford station

22400 West Seven Mile Road

Detroit, Michigan 48219

American Fishing Tackle Manufacturers Association

AFTMA

20 North Wacker Drive

Chicago, Illinois 60606

American Gas Association

1515 Wilson Boulevard

Arlington, Virginia 22209

American Leather Chemists Association

ALCA

c/o University of Cincinnati

Cincinnati, Ohio 45221

American National Standards Institute, Inc.

ANSI

1430 Broadway

New York, New York 10018

American Society for Testing and Materials

ASTM

1916 Race Street

Philadelphia, Pennsylvania 19103

American Society of Heating, Refrigerating and Afr-Condtioning Engineers

ASHRAE

345 East 47 th Street

New York, New York 10017

American Society of Mechanical Engineers

ASME

345 East 47 th Street

New York, New York 10017

American Society of Sanitary Engineering

ASSE

228 Standard Building

Cleveland, Ohio 44113

American Water Works Association, Inc.

AWWA

2 Park Avenue

New York, New York 10016 
Spartanburg, South Carolina 29301

Archery Manufacturers Organization

AMO

618 Chalmers Street

Flint, Michigan 48503

Architectural Aluminum Manufacturers Association

AAMA

410 N. Michigan Avenue

Suite 960

Chicago, Illinois 60611

Architectural Woodwork Institute

AWI

Chesterfield House

Suite "A"

5055 S. Chesterfield Road

Arlington, Virginia 22206

Asphalt and Vinyl Asbestos Tile Institute

101 Park Avenue

New York, New York 10017

Association of Bedding and Furniture Law Officials

ABFLO

270 Broadway

New York, New York 10007

Association of Home Appliance Manufacturers

AHAM

20 North Wacker Drive

Chicago, Illinois 60606

Association of Official Analytical Chemists

AOAC

P.0. Box 540

Benjamin Franklin Station

Washington, D.C. 20004

Battery Council Intermational

$\mathrm{BCI}$

1801 Murchison Drive

Burlingame, California 94010

Bicycle Manufacturers Association

BMA

122 East 42nd Street

New York, New York 10017

Billiard Congress of America

$\mathrm{BCA}$

717 N. Michigan Avenue

Chicago, Illinois 60611

Boating Industry Association

BIA

333 N. Michigan Avenue

Chicago, Illinois 60601

Builders Hardware Manufacturers Association

BHMA

60 East 42nd Street

New York, New York 10017

Can Manufacturers Institute, Inc.

CMI

821 15th Street, NW.

Washington, D.C. 20005

Chain Link Fence Manufacterers Institute

One Stone Place

Box 515

Bronxville, New York 10708 
Chemical Specialties Manufacterers Association

50 East 4 lst Street

New York, New York 10017

Composite Cen and Tube Institute

1725 Eye Street, NW.

Washington, D.C. 20006

Compressed Air and Gas Institute

122 East 42nd Street

New York, New York 10017

Cordage Institute

2300 Calvert Street, NW.

Washington, D.C. 20008

Cosmetic, Toiletry and Fragrance Association, Inc.
1625 Eye Street, NW.

Washington, D.C. 20006

Edison Electric Institute

90 Park Avenue

New York, New York 10016

$20 \mathrm{Cl}$ Eye Street, NW.

Washington, D.C. 20006

1151 Boston-Providence Turnpike

Norwood, Massachusetts 02062

Fir and Hemlock Door Association

Yeon Building

Portland, Oregon 97204

Glass Tempering Association

1325 Topeka Avenue

Topeka, Kansas 66612

Gypsum Association

201 North Wells Street

GA

Room 2510

Chicago, Illinois 60606

Hardwood Plywood Menufacturers Association

P.0. Box 6246

HPMA

3210 South Walter Reed Drive

Arlington, Virginia 22206

Hearing Aid Industry Conference, Inc.

Ste. 628

HAIC

1101 Connecticut Avenue, NW.

Washington, D.C. 20036

Incinerator Institute of America

One Stone Place

Bronxville, New York 10708

Industrial Fasteners Institute

1505 East Ohio Building

IFI

1717 East Ninth Street

Cleveland, Ohio 44114

Institute of Electrical and Electronics Engineers, Inc.
345 East 47 th Street

IEEE

Institute of High Fidelity, Inc.

516 Fifth Avenue

IHF

New York, New York 10036 
International Comission on Rules for the Approval of Electrical Equipment

International Electrotechnical Comission

1 rue de Varembe

Geneva, Switzerland

International Organization for Standardization

1 rue de Varembe"

Geneva, Switzerland

(

Maple Flooring Manufacturers Association

MF'MA

424 Washington Avenue

Oshkosh, Wisconsin 54901

Mechanical Contractors Association of America, Ine.

MCAA

Suite 750

5530 Wisconsin Avenue, NW.

Washington, D.C. 20015

Motorcycle Industry Council

MIC

1001 Connecticut Avenue, NW.

Washington, D.C. 20036

Metal Ladder Manufacturers Association

MLMA

Box 580

Greenville, Pennsylvania 16125

National Association of Architectural Metal Manufacturers

Suite 500

1010 West Lake Street

Oak Park, Illinois 60301

National Association of Furniture Manufacturers, Inc.

NAFM

666 Lake Shore Drive, Room 1727

Chicago, Illinois 60611

National Association of Plastic Fabricators, Ine.

NAPF

4720 Montgomery Lane

Washington, D.C. 20014

National Association of Rocketry

NAR

1891 Massachusetts Avenue

McLean, Virginia 22101

National Builders' Hardware Association

NBKA

1290 Avenue of the Americas

New York, New York 10019

National Concrete Masonry Association

NCMA

P.0. Box 9185

Rosslyn Station

Arlington, Virginia 22209

National Council on Radiation Protection and Measurements

NCRPM

7910 Woodmont Avenue

Suite 1016

Washington, D.C. 20014

National Electrical Manufacturers Association

NEMA

155 East 44 th Street

New York, New York 10017 
Canton, Ohio 44708

National Oak Flooring Manufacturers Association, Inc.

NOFMA

814 Sterick Building

Memphis, Tennessee 38103

National Paper Box Association

NPBA

231 Kings Highway East

Haddonfield, New Jersey 08033

National Particleboard Association

NPA

2306 Perkins Place

Silver Spring, Maryland 20910

National Sanitation Foundation

NSF

NFS Building

Ann Arbor, Michigan 48105

National Soft Drink Association

NSDA.

1128 16th Street, NW.

Washington, D.C. 20036

National Swimming Pool Institute

NSPI

$2000 \mathrm{~K}$ Street, NW.

Washington, D.C. 20006

National Terrazzo and Mosaic Association, Inc.

NTMA

716 Church Street

Alexandria, Virginia 22314

National Woodwork Manufacturers Association

NWMA

400 West Madison Street

Chicago, Illinois 60606

Packaging Institute, Inc.

342 Madison Avenue

New York, New York 10017

Paper Stationery and Tablet Manufacturers Association

PSTMA

Room 2301

444 Madison Avenue

New York, New York 10022

Paperboard Packaging Council

PPC

1250 Connecticut Avenue, IW.

Washington, D.C. 20036

Pressure Sensitive Tape Council

PSTC

1201 Waukegan Road

Glenview, Illinois 60025

Recording Industry Association of America, Inc.

1 East 57th Street

New York, New York 10022 
Red Cedar Shingle and Handsplit Shake Bureau

5510 White Building

Seattle, Washington 98101

Rubber Manufacturers Association

RMA

444 Madison Avenue

New York, New York 10022

Safety Helmet Council of America

SHCA

9107 Wilshire Boulevard

Suite 506

Beverly Hills, California 90210

Screen Manufacturers Association

SCRMA

110 North Wacker Drive

Chicago, Illinois 60606

Sealed Insulating Glass Manufacturers Association SIGMA

P.O. Box 374

Carpentersville, Illinois 60110

Sheet Metal and Air Conditioning Contractors National Association, Inc.

SMACNA

1611 North Kent Street

Arlington, Virginia 22209

Snell Memorial Foundation

SMF

5 Lake Shore Close

North Tarrytown, New York 10591

Society of Automotive Engineers, Inc.

SAE

Two Pennsylvania Plaza

New York, New York 10001

Society of the Plastics Industry, Inc.

SPI

250 Park Avenue

New York, New York 10017

Specialty Wire Association

SWA

1625 Eye Street, NW.

Washington, D.C. 20006

Steel Door Institute

STDI

2130 Keith Building

Cleveland, Ohio 44115

Steel Window Institute

SWI

2130 Keith Building

Cleveland, Ohio 44115

Sump Pump Manufacturers Association

SPMA

221 North LaSalle Street

Chicago, Illinois 60601

Technical Association of the Pulp and Paper Industry

TAPPI

One Dunwoody Park

Atlanta, Georgia 30341

Underwriters' Laboratories, Inc.

UL

207 East Ohio Street

Chicago, Illinois 60611

U.S. Department of Commerce

USC

Office of Engineering Standards Services

National Bureau of Standards

Washington, D.C. 20234

Vacuum Cleaner Manufacturers Association

VCMA

1615 Collamer

Cleveland, Ohio 44110 
Water Conditioning Foundation

1780 Maple Street

Northfield, Illinois 60093

Water Systems Council

221 North IaSalle Street

WSC

Chicago, Illinois 60601

Western Wood Products Association

700 Yeon Building

Portland, Oregon 97204

WWPA

Wood and Synthetic Flooring Institute of America

1441 Shermer Road.

WSFI

Northbrook, Illinois 60062 
4. TITLE AND SUBTITLE

Tabulation of Voluntary Standards and Certification Programs for Consumer Products

March 1973

5. Publication Date

6. Performing Organization Code

8. Performing Organization

Sophie J。 Chumas

7. AUTHOR(S)

9. PERF ORMING ORGANIZATION NAME AND ADDRESS

NATIONAL BUREAU OF ST ANDARDS

DEPARTMENT OF COMMERCE

WASHINGTON, D.C. 20234

10. Project/Task/Work Unit No.

11. Contract/Grant No.

3. Type of Report \& Period Covered

Final

12. Sponsoring Organization Name and Address

Same as item 9 .

14. Sponsoring Agency Code

\section{SUPPLEMENTARY NOTES}

ABSTRACT (A 200-word or less factual summary of most significant information. If document includes a significant bibliography or literature survey, mention it here.)

This tabulation is a revised and enlarged version of NBS Technical Note 705, "Tabulation of Voluntary National Standards, Industry Standards, International Recommendations and Certification Programs for Consumer Products," issued December 1971 。

Technical Note 705 was a revision of the Draft Tabulation compiled by the American National Standards Institute and issued in April, 1970. The categories and products covered in the tabulation were originally based on those listed in the Consumer Product Safety Index (CPSI) of the National Commission on Product Safety, published in July 1970. The products and categories in the current revision are based on those developed for the National Electronic Injury Surveillance System (NEISS) of the Food and Drug Administration. The NEISS listings are an expansion and revision of the CPSI. NEISS product areas were slightly altered to conform to the scope of this tabulation.

The tabulation lists over 700 product areas and over 1000 standards titles covering products found in and around the home. (The major consumer product areas not included are foods, beverages, and drugs.) The tabulation also indicates the applicable voluntary national, industrial, and international standards which deal primarily with either safety or performance or both aspects of the products listed. For some of the product areas, there are no applicable standards. Available information on certification programs and standards under development, and the Standard Industrial Classification (SIC) numbers for the products are also provided.

17. KEY WORDS (Alphabetical order, separated by semicolons) Certification programs; consumer products; household products; industry standards; international recommendations; national standards; product standards; recommended practices; specifications; test methods.

\begin{tabular}{|c|c|c|}
\hline $\begin{array}{l}\text { 18. AV AILABILITY ST ATEMENT } \\
\mathrm{x} \text { UNL IMIT ED. }\end{array}$ & $\begin{array}{l}\text { 19. SECURITY CLASS } \\
\text { (THIS REPORT) } \\
\text { UNCL ASSIFIED }\end{array}$ & $\begin{array}{l}\text { 21. NO. OF PAGES } \\
119\end{array}$ \\
\hline $\begin{array}{l}\square \text { FOR OFFICIAL DISTRIBUTION. DO NOT RELEASE } \\
\text { TO NTIS. }\end{array}$ & $\begin{array}{l}\text { 20. SECURITY CLASS } \\
\text { (THIS PAGE) } \\
\text { UNCL ASSIFIED }\end{array}$ & $\begin{array}{l}\text { 22. Price } \\
\$ 1.25 \text { Domestic Post- } \\
\text { paid: } \$ 1.00 \mathrm{GPO} \\
\text { Bookstore }\end{array}$ \\
\hline
\end{tabular}



PERIODICALS

JOURNAL OF RESEARCH rcports National Bureau of Standards research and development in physics, mathematics, and chemistry. Comprehensive scientific papers give complete details of the work, including laboratory data, experimental procedures, and theoretical and mathcmatical analyses. Illustrated with photographs, drawings, and charts. Includes listings of other NBS papers as issued.

Published in two sections, available separately:

\section{- Physics and Chemistry (Section A)}

Papers of interest primarily to scientists working in these fields. This section covers a broad range of physical and chemical research, with major emphasis on standards of physical measurement, fundamental constants, and properties of matter. Issued six times a year. Annual subscription: Domestic, $\$ 17.00$; Foreign, \$21.25.

\section{- Mathematical Sciences (Section B)}

Studies and compilations designed mainly for the mathematician and theoretical physicist. Topics in mathematical statistics, theory of experiment design, numerical analysis, theoretical physics and chemistry, logical design and programming of computers and computer systems. Short numerical tables. Issued quarterly. Annual subscription: Domestic, $\$ 9.00$; Foreign, $\$ 11.25$.

\section{TECHNICAL NEWS BULLETIN}

The best single source of information concerning the Bureau's measurement, research, developmental, cooperative, and publication activities, this monthly publication is designed for the industry-oriented individual whose daily work involves intimate contact with science and technology-for engineers, chemists, physicists, research managers, product-development managers, and company cxecutives. Includes listing of all NBS papers as issued. Annual subscription: Domestic, $\$ 6.50$; Foreign, $\$ 8.25$.
NONPERIODICALS

Applied Mathematics Series. Mathematical tables, manuals, and studies.

Building Science Series. Research results, test methods, and performance criteria of building materials, components, systems, and structures.

Handbooks. Recommended codes of engineering and industrial practice (including safety codes) developed in cooperation with interested industries, professional organizations, and regulatory bodies.

Special Publications. Proceedings of NBS conferences, bibliographies, annual reports, wall charts, pamphlets, etc.

Monographs. Major contributions to the technical literature on various subjects related to the Bureau's scientific and technical activities.

National Standard Reference Data Series. NSRDS provides quantitative data on the physical and chemical properties of materials, compiled from the world's literature and critically evaluated.

Product Standards. Provide requirements for sizes, types, quality, and methods for testing various industrial products. These standards are developed cooperatively with interested Government and industry groups and provide the basis for common understanding of product characteristics for both buyers and sellers. Their use is voluntary.

Technical Notes. This series consists of communications and reports covering both other-agency and NBS-sponsored work) of limited or transitory interest.

Federal Information Processing Standards Publications. This series is the official publication within the Federal Government for information on standards adopted and promulgated under the Public Law 89-306, and Bureau of the Budget Circular A-86 entitled, Standardization of Data Elements and Codes in Data Systems.

Consumer Information Series. Practical information, based on NBS research and experience, covering areas of interest to the consumer. Easily understandable language and illustrations provide useful background knowledge for shopping in today's technological marketplacc.

\section{BIBLIOGRAPHIC SUBSCRIPTION SERVICES}

The following current-awareness and literature-survey bibliographies are issued periodically by the Bureau :

Cryogenic Data Center Current Awareness Service (Publications and Reports of Interest in Cryogenics). A literature survey issued weekly. Annual subscription: Domestic, $\$ 20.00$; foreign, $\$ 25.00$.

Liquefied Natural Gas. A literature survey issued quarterly. Annual subscription: $\$ 20.00$.

Superconducting Devices and Materials. A literature survey issued quarterly. Annual subscription: $\$ 20.00$. Send subscription orders and remittances for the preceding bibliographic services to the U.S. Department of Commerce, National Technical Information Service, Springfield, Va. 22151.

Electromagnetic Metrology Current Awareness Service (Abstracts of Selected Articles on Measurement Techniques and Standards of Electromagnetic Quantities from D-C to Millimeter-Wave Frequencies). Issued monthly. Annual subscription: $\$ 100.00$ (Special rates for multi-subscriptions). Send subscription order and remittance to the Electromagnetic Metrology Information Center, Electromagnetics Division, National Bureau of Standards, Boulder, Colo. 80302.

Order NBS publications (except Bibliographic Subscription Services)

from: Superintendent of Documents, Gorcrnment Printing Office, Wash- 
U.S. DEPARTMENT OF COMMERCE

National Bureau of Standards

Washington, D.C. 20234

DFFICIAL BUSINESS

Penalty for Private Use, \$3DD
POSTAGE AND FEES PAID U.S. DEPARTMENT OF COMMERCE 215

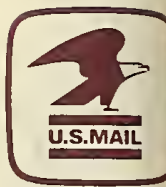

